\title{
DISTRIBUTED FIBER OPTIC INTRUSION SENSOR SYSTEM FOR MONITORING LONG PERIMETERS
}

\author{
A Dissertation \\ by \\ JUAN C. JUAREZ \\ Submitted to the Office of Graduate Studies of \\ Texas A\&M University \\ in partial fulfillment of the requirements for the degree of \\ DOCTOR OF PHILOSOPHY
}

August 2005

Major Subject: Electrical Engineering 


\title{
DISTRIBUTED FIBER OPTIC INTRUSION SENSOR SYSTEM FOR MONITORING LONG PERIMETERS
}

\author{
A Dissertation \\ by \\ JUAN C. JUAREZ \\ Submitted to the Office of Graduate Studies of \\ Texas A\&M University \\ in partial fulfillment of the requirements for the degree of \\ DOCTOR OF PHILOSOPHY
}

Approved by:

Chair of Committee, Henry F. Taylor

Committee Members, Chin B. Su

Jose Silva-Martinez

Ray W. James

Head of Department, Chanan Singh

August 2005

Major Subject: Electrical Engineering 


\author{
ABSTRACT \\ Distributed Fiber Optic Intrusion Sensor System \\ For Monitoring Long Perimeters. (August 2005) \\ Juan C. Juarez, B.S., Texas A\&M University; \\ M.S., Texas A\&M University \\ Chair of Advisory Committee: Dr. Henry F. Taylor
}

A distributed sensor using an optical fiber for detecting and locating intruders over long perimeters $(>10 \mathrm{~km})$ is described. Phase changes resulting from either the pressure of the intruder on the ground immediately above the buried fiber or from seismic disturbances in the vicinity are sensed by a phase-sensitive optical time-domain reflectometer $(\phi-\mathrm{OTDR})$. Light pulses from a cw laser operating in a single longitudinal mode and with low (MHz/min range) frequency drift are injected into one end of the single mode fiber, and the backscattered light is monitored with a photodetector. In laboratory tests with $12 \mathrm{~km}$ of fiber on reels, the effects of localized phase perturbations induced by a piezoelectric fiber stretcher on $\phi-$ OTDR traces were characterized. In field tests in which the sensing element is a single mode fiber in a 3-mm diameter cable buried in an 8 to 18 inch deep, 4 inch wide trench in clay soil, detection of intruders on foot up to $15 \mathrm{ft}$ from the cable line was achieved. In desert terrain field tests in which the sensing fiber is in a 4.5-mm diameter cable buried in a $1 \mathrm{ft}$ deep, $2.5 \mathrm{ft}$ wide trench filled with loose sand, high sensitivity and consistent detection of intruders on foot and of 
vehicles traveling down a road near the cable line was realized over a cable length of 8.5 $\mathrm{km}$ and a total fiber path of $19 \mathrm{~km}$ in real time. In a final series of field tests in clay soil, phase changes produced by the steps of a person walking up to $15 \mathrm{ft}$ away from the buried cable were observed, and vehicles traveling at $10 \mathrm{mph}$ were consistently detected up to $300 \mathrm{ft}$ away. Based on these results, this technology may be regarded as a candidate for providing low-cost perimeter security for nuclear power plants, electrical power distribution centers, storage facilities for fuel and volatile chemicals, communication hubs, airports, government offices, military bases, embassies, and national borders. 
To My Loving Mother

and

The Memory of My Father 


\section{ACKNOWLEDGEMENTS}

I would like to express my gratitude and sincere appreciation to Dr. Henry F. Taylor, the chairman of my advisory committee, for his continuous guidance and support during my research work. His generosity, patience, and profound knowledge of the field have always amazed me and will always hold my respect. I would also like to thank Dr.

Chin B. Su, Dr. Jose Silva-Martinez, and Dr. Ray W. James for volunteering their time to serve as members of my graduate committee. My appreciation is also extended to Robert Atkins for his work in the laboratories and his active cooperation in this research. Special thanks go to Dr. Kyoo Nam Choi, Taehan Bae, Kyongtae Park, Xiaoke Wan, Eric Maier, Hicham Fadel, Harini Kuppuswamy, Bilal Malik, Andrew Conkey, and Colleen Dominick for their helpful discussions, aid, and support.

Special thanks go to my parents for their always-present love, support, and encouragement. My appreciation also goes to my family and friends for always being there for me. Finally, I thank God. 


\section{TABLE OF CONTENTS}

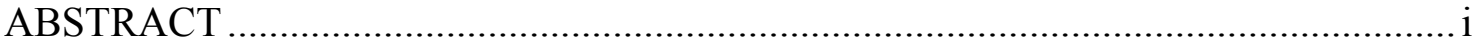

ACKNOWLEDGEMENTS .............................................................................. vi

TABLE OF CONTENTS ....................................................................................... vii

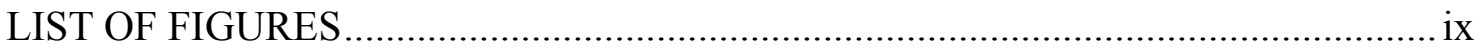

CHAPTER

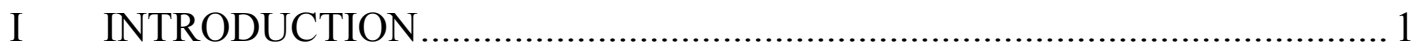

II THEORETICAL BACKGROUND ................................................... 6

A. Rayleigh Scattering in a Single Mode Fiber ........................................ 6

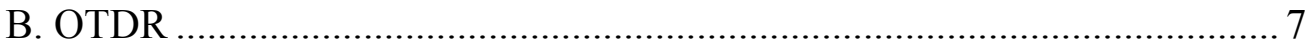

C. Phase-Sensitive OTDR ...................................................................... 10

D. Polarization of Rayleigh Backscattering ................................................ 14

E. Pressure Response of a Buried Cable ..................................................... 15

F. Simulation of $\phi$-OTDR Response to a Phase Perturbation ....................... 17

G. Frequency Drift Measurement.................................................................. 19

III ERBIUM DOPED FIBER LASER CHARACTERIZATION....................... 22

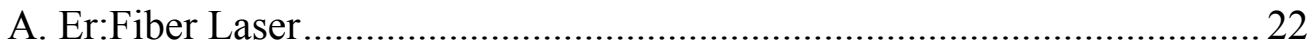

B. Linewidth Measurements ................................................................... 25

C. Frequency Drift Measurements ................................................................ 26

IV OTDR LABORATORY EXPERIMENTS …........................................... 30

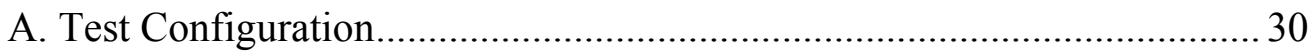

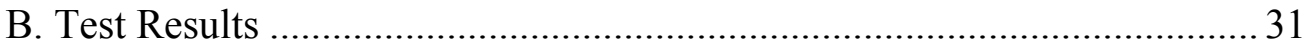

V FIELD TESTS, SERIES 1, BRAZOS COUNTY, TX ................................ 36

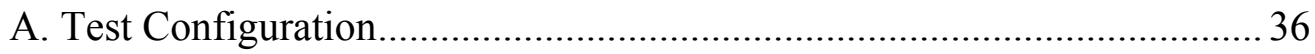

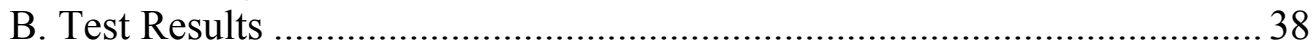


CHAPTER Page

VI FIELD TESTS, SERIES 2, YUMA, AZ ................................................ 43

A. Test Configuration................................................................... 43

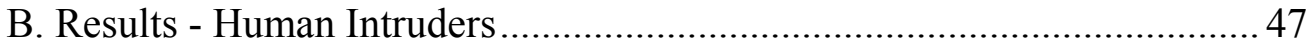

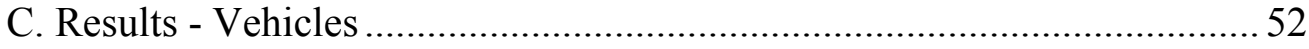

VII FIELD TESTS, SERIES 3, BRAZOS COUNTY, TX ................................ 54

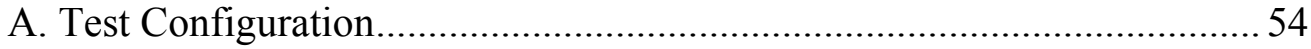

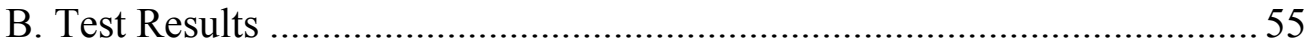

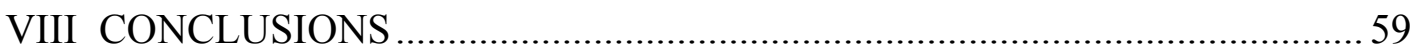

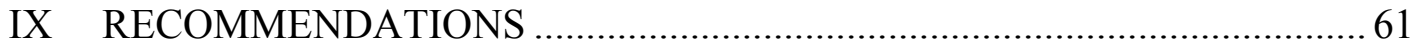

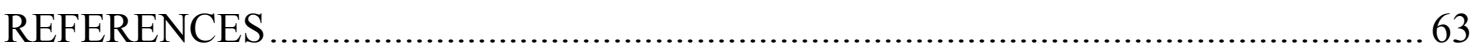

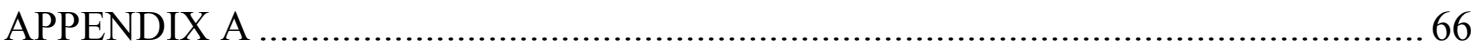

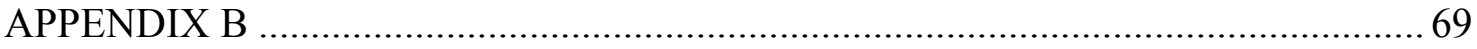

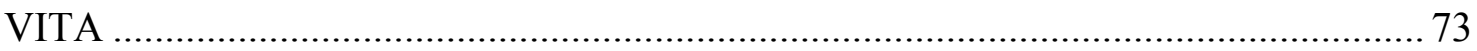




\section{LIST OF FIGURES}

FIGURE

1. Phase-sensitive OTDR ( $\phi$-OTDR) used for intrusion sensing 4

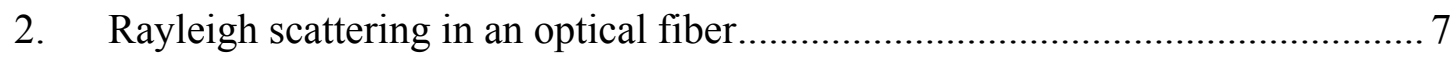

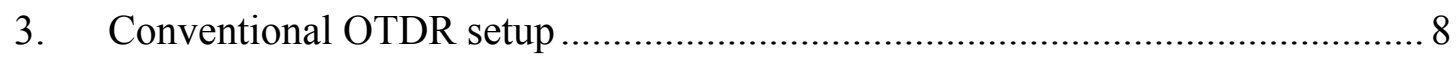

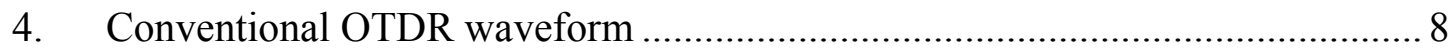

5. Interference of backscattered light arising from an optical pulse ..................... 12

6. Fabry-Perot interferometer in an optical fiber........................................... 13

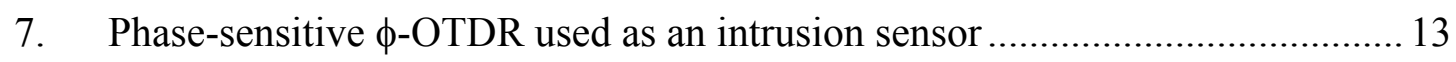

8. Simulation result showing effect of a localized $\pi / 4$ phase perturbation on the temporal dependence of Rayleigh backscattered power. The upper and lower traces have been vertically displaced by $\pm 100 \mathrm{nW}$ so that the curves can easily be distinguished from one another.

9. Simulation result for dependence of range resolution on range in the fiber.

10. Mach-Zehnder test setup for frequency drift measurements.

11. Previously researched fiber laser setup with short and long optical

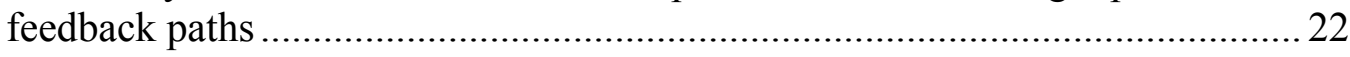

12. Experimental setup for fiber laser used in the intrusion sensor system ............ 24

13. Spectral linewidth scan of Erbium doped fiber laser ................................... 24

14. Delayed self-heterodyne test setup for instantaneous linewidth measurements

15. Delayed self-heterodyne measurement of laser linewidth

16. Laser frequency drift monitoring setup incorporating two MachZehnder interferometers 
17. Laser frequency drift with: (a) environmental disturbances at 2:52:03 PM. (b) normal laboratory conditions at 8:36:44 AM. (c) minimal disturbances at 10:32:04 PM ................................................................... 28

18. Laboratory setup for characterizing the $\phi$-OTDR system .................................. 30

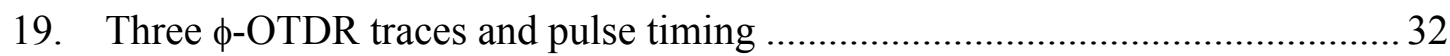

20. Effect of a phase perturbation on successive $\phi$-OTDR traces .......................... 33

21. (a) Consecutive OTDR traces superimposed with phase changes at 2 $\mathrm{km}$ location. (b) Differences of OTDR traces superimposed with phase changes at $2 \mathrm{~km}$ location. (c) Magnitudes of $2 \mathrm{~km}$ location plotted vs. applied phase shifter voltage.

22. Preliminary field tests setup in clay soil for characterizing the $\phi$-OTDR system.

23. Configuration of buried sensor. 38

24. $\phi$-OTDR traces acquired before and after an $80 \mathrm{~km}$ person has stepped on the ground above the cable

25. (a) Superposition of $\phi$-OTDR traces produced in response to a person walking on the ground above the buried cable; (b) difference plots of the same data.

26. Response of $\phi$-OTDR over a time period of 2 seconds at ranges of 2,3 , and $4 \mathrm{~km}$ when a person is walking on the ground above the buried fiber cable at the $2 \mathrm{~km}$ location.

27. Field tests setup in desert terrain for characterizing the $\phi$-OTDR system

28. Experimental setup for fiber laser used as the light source in Yuma, AZ field tests

29. Sample screen capture of PC with both OTDR traces and processed signal 
30. Results of an intruder taking a step at $5.4 \mathrm{~km}$ range. As indicated by the labels on the video frames, the elapsed time between (a) and (b) is $9 / 30$ $\mathrm{s}(0.30 \mathrm{~s})$ and between (b) and (c) is $8 / 30 \mathrm{~s}(0.27 \mathrm{~s})$. The effect of the intruder on the loop-back portion of the fiber at a range of $13.6 \mathrm{~km}$ is not detected in this case.

31. Response to an intruder taking a step at a range of $3.3 \mathrm{~km}$. The loopback signal is seen in the white processed signal waveform at a range of $17.7 \mathrm{~km}$

32. Response to an intruder taking a step at a range of $5.4 \mathrm{~km}$ with a strong signal in the outbound fiber....

33. Response to an intruder taking a step at a range of $5.4 \mathrm{~km}$ with a strong signal in the inbound fiber.

34. Response to automobile traveling on road $8^{\prime}-20^{\prime}$ from the buried sensor.

35. Field tests setup in clay soil for characterizing the $\phi$-OTDR system 54

36. (a) and (b) $\phi$-OTDR traces for both orthogonal polarizations acquired before and after an $80 \mathrm{~km}$ person has stepped on the ground above the cable along with the difference

37. Setup for lateral tests.

38. Temporal response of $\phi$-OTDR over 12 seconds at ranges of 1,2 , and 3 $\mathrm{km}$ when a person is approaching the buried fiber cable at the $2 \mathrm{~km}$ location from $15 \mathrm{ft}$ away. 


\section{CHAPTER I}

\section{INTRODUCTION}

The optical time domain reflectometer (OTDR), initially demonstrated over two decades ago [1-3], is now widely used for locating breaks and other anomalies in fiber optic links and networks. In an OTDR system, light pulses from a semiconductor laser are injected into one end of a fiber, and Rayleigh backscattered light returned from the fiber is monitored with a photodetector. The system detects the presence and location of perturbations which affect the intensity of the light returned from the fiber, but does not in general respond to phase modulation of the light. The spectral width of the modulated laser is very broad (GHz to $\mathrm{THz}$ range), so that fluctuations in the return signal due to interference of backscattered components from different parts of the fiber are for the most part avoided. When present to a noticeable extent, coherent effects represent an undesirable source of noise in an OTDR trace.

The distributed sensor described in this research utilizes a phase-sensitive OTDR ( $\phi$-OTDR) system designed to enhance coherent effects rather than avoid them [4]. Phase sensitivity results from interference of the light backscattered from different parts of the fiber which arrive simultaneously at the photodetector. As a practical matter, the $\phi$-OTDR can detect perturbations much too small to be perceived with a conventional OTDR system.

This dissertation follows the style of IEEE Journal of Lightwave Technology. 
In prior research, the $\phi$-OTDR has been applied with both pulsed and cw laser light sources to detect and determine the location of phase perturbations caused by stretching or heating optical fibers. A repetitively pulsed Q-switched yttrium aluminum garnet laser was the light source in a system for observing a piezoelectrically induced length change, and a pulsed semiconductor laser was used in the detection of a rapidly increasing temperature [5]. A single frequency $\mathrm{cw}$ semiconductor laser in conjunction with an external acoustooptic modulator was used to sense a localized thermal perturbation [6,7], and an Er:fiber laser in combination with an electrooptic modulator was applied to observe a piezoelectrically induced length change [8].

Other distributed sensors applicable for intrusion sensing are based on the Sagnac interferometer. The first reported was a two-interferometer configuration incorporating a Mach-Zehnder to measure phase-change rate with the Sagnac interferometer to detect non-reciprocal phase perturbances [9]. Modified versions have been proposed to eliminate the position sensitivity of the Sagnac interferometer with a phase-shifter or the need for a highly coherent light source with wavelength division multiplexing (WDM) techniques [10]. A frequency modulation continuous wave technique was applied to a birefringent Sagnac loop to use the intensity and frequency of a beat signal produced by two forward-coupled mode beams to determine the amplitude and location of an applied stress, respectively [11]. A dual-wavelength, merged Sagnac and Michelson interferometer system has been proposed with a broadband source for optimal operation of the Sagnac [12]. Two-loop and variable-loop Sagnac interferometer systems have been investigated to preserve the inherent insensitivity to reciprocal disturbances of the 
Sagnac $[13,14]$. A single source, single detector WDM system incorporating dual 40-km Sagnac loops has been applied with real-time location of multiple time-varying disturbances [15].

In the distributed intrusion sensor reported here, the phase changes of interest result from the pressure of an intruder on the ground above the buried fiber cable $[4,16]$, as illustrated in Figure 1. Light pulses from a cw laser are gated into one end of the fiber via a pulsed intensity modulator, and the backscattered light from the fiber is monitored with a photodetector. As with the conventional OTDR, the $\phi$-OTDR trace is a plot of returned optical power vs. time. When the sensing fiber and light source are stabilized, the resulting trace exhibits a unique temporal signature characteristic of the state of the sensor. The effect of phase changes resulting from the pressure of a person on the ground immediately above or near the buried fiber are sensed by subtracting a $\phi$-OTDR trace from an earlier stored trace. The time at which changes in the $\phi$-OTDR trace occur are proportional to the range (distance along the fiber from the proximal end) at which the phase perturbation is applied. The spatial resolution, $\Delta \mathrm{z}$, of the sensor is determined by the width of the pulses, $T_{p}$, gated into the fiber such that, $\Delta z=c T_{p} / 2 n_{g}$, where $c$ is the speed of light in a vacuum and $\mathrm{n}_{\mathrm{g}}$ is the group refractive index [17].

In contrast to the conventional OTDR, the $\phi$-OTDR used for intrusion sensing requires a laser with minimal frequency drift as well as narrow instantaneous linewidth. Low frequency drift is critical because frequency modulation of the laser causes trace-totrace fluctuations in the $\phi$-OTDR waveform - a source of noise which obscures the effect of an intruder. Narrow linewidth is necessary to cause backscattered fields within the 
pulse width to interfere at the photodetector and achieve the phase sensitivity that is necessary for the $\phi$-OTDR.

The Er:fiber laser was selected for this application because it emits in the spectral region where silica fiber losses are a minimum, it can be used with Er:fiber amplifiers to achieve high average and pulsed power levels, and it can emit in a single longitudinal mode for narrow linewidth operation [8].

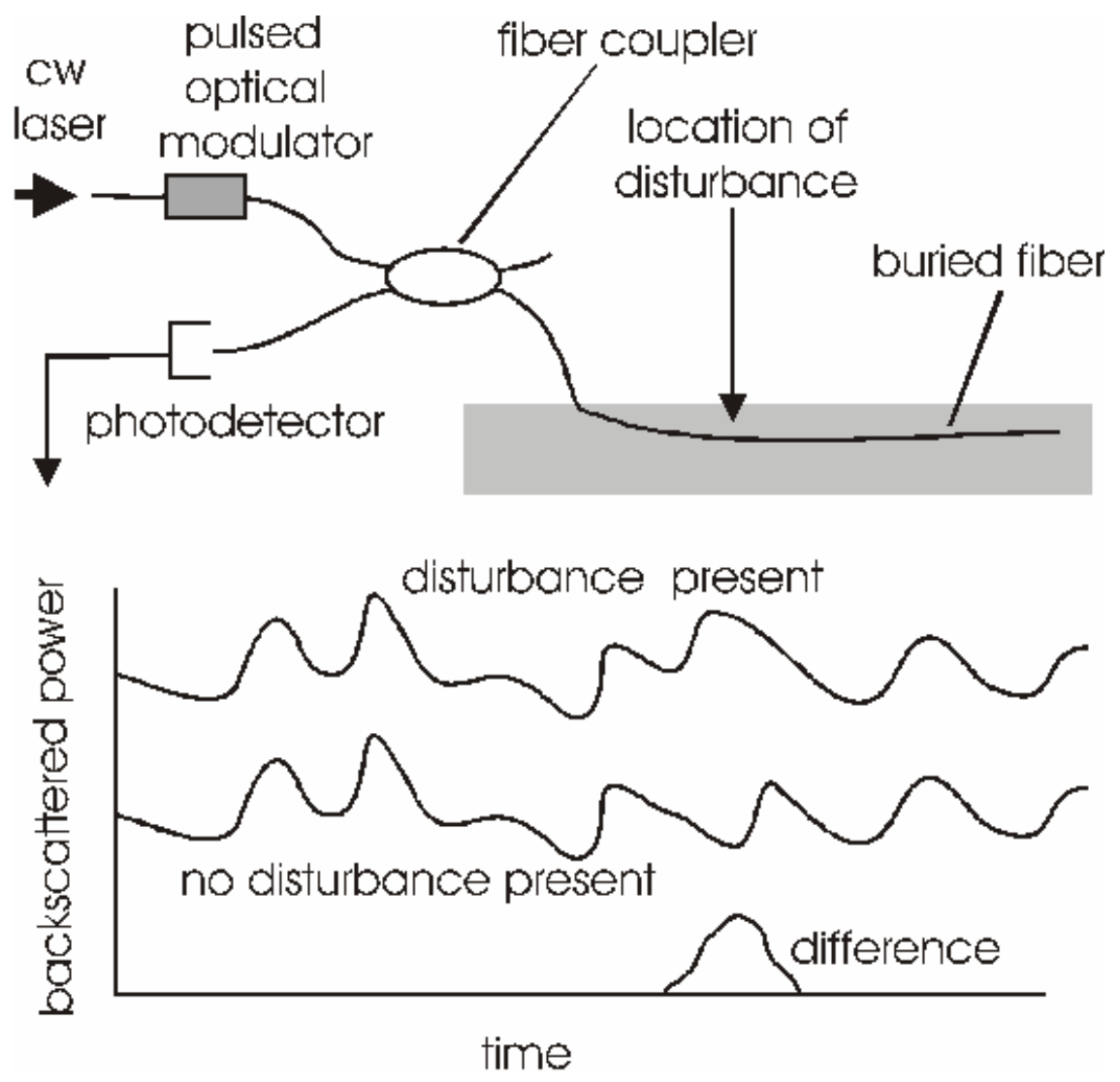

Figure 1. Phase-sensitive OTDR ( $\phi$-OTDR) used for intrusion sensing 
This dissertation describes laboratory characterization of a spectrally stable Erbium doped fiber laser for use in a $\phi$-OTDR system, followed by laboratory characterization of a $\phi$-OTDR system itself and field testing of an intrusion sensing system using a buried cable in clay soil and sand. In laboratory experiments with fiber on reels, the effects of controlled phase perturbations induced by a piezoelectric fiber stretcher on $\phi$-OTDR traces were characterized. In the field tests in clay soil, intruders were observed walking up to $15 \mathrm{ft}$ away from the buried fiber cable line and phase shifts of several- $\pi$ radians were observed when walking on the ground directly above the buried cable. In the desert terrain field tests, people walking on the ground over or near the buried fiber cable and vehicles traveling down a road near the cable were consistently detected. 


\section{CHAPTER II}

\section{THEORETICAL BACKGROUND}

\section{A. Rayleigh Scattering in a Single Mode Fiber}

When optical fiber is drawn from its molten state, microscopic variations arise in the density of the material as it cools, which in turn produces variations in the index of refraction of the fiber. This causes scattering of optical power known as Rayleigh backscattering, which has become the dominant loss mechanism in single mode fibers accounting for nearly $96 \%$ of the attenuation in today's fibers [18].

The inhomogeneities in the index of refraction of a fiber can be modeled as scattering centers embedded in a homogeneous material with sizes much smaller than those of the optical wavelengths [19]. As an optical wave travels along the fiber, light will scatter in all directions, but a small fraction of the scattered light will couple into the fiber core and propagate in the reverse direction as shown in Figure 2. This phenomenon is commonly used in optical time domain reflectometry (OTDR) to monitor intensity attenuations throughout a fiber length. Rayleigh scattering loss is proportional to $\lambda^{-4}$ with increasing wavelength throughout the visible and near-infrared spectral regions to a minimum of about $0.2 \mathrm{~dB} / \mathrm{km}$ at $1550 \mathrm{~nm}$. Beyond this wavelength the attenuation increases rapidly due to optical absorption resulting from the excitation of phonons in the fiber material. 


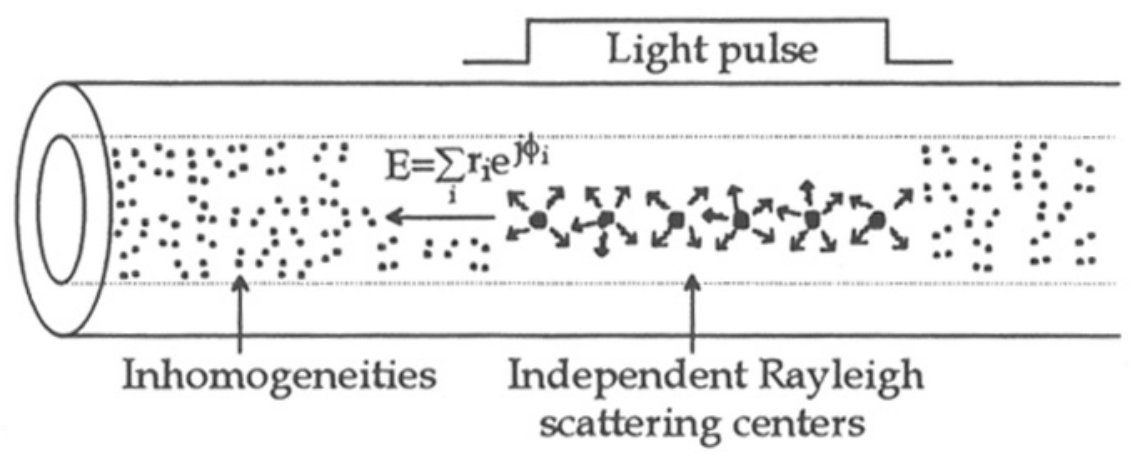

Figure 2. Rayleigh scattering in an optical fiber

\section{B. OTDR}

Optical time domain reflectometry (OTDR) is a common technique for nondestructive characterization of attenuation and imperfections in long lengths of fiber especially when only one end of the fiber is available for measurement. Some applications of this technique include measurements of splice loss, connector loss, microbending loss, diameter fluctuation, fiber length, differential modal scattering gain, and mode conversion at joints [20]. It can also be used for sensing perturbances created by magnetic fields, stress, strain, and temperature changes that affect the propagation of light in the fiber [21].

A typical OTDR configuration consisting of a pulse generator, a laser, a directional coupler, a photodetector, and a signal processor is illustrated in Figure 3. The modulated laser light is gated into the fiber under test via the directional coupler. Rayleigh backscattered light propagates backwards to the photodetector via the same coupler and produces a continuous signal from all points along the fiber. The detected 
optical power will decrease exponentially with respect to the distance along the fiber and show abrupt peaks and dips at the location of splices, connectors, and breaks due to Fresnel reflections as shown in Figure 4 [19].

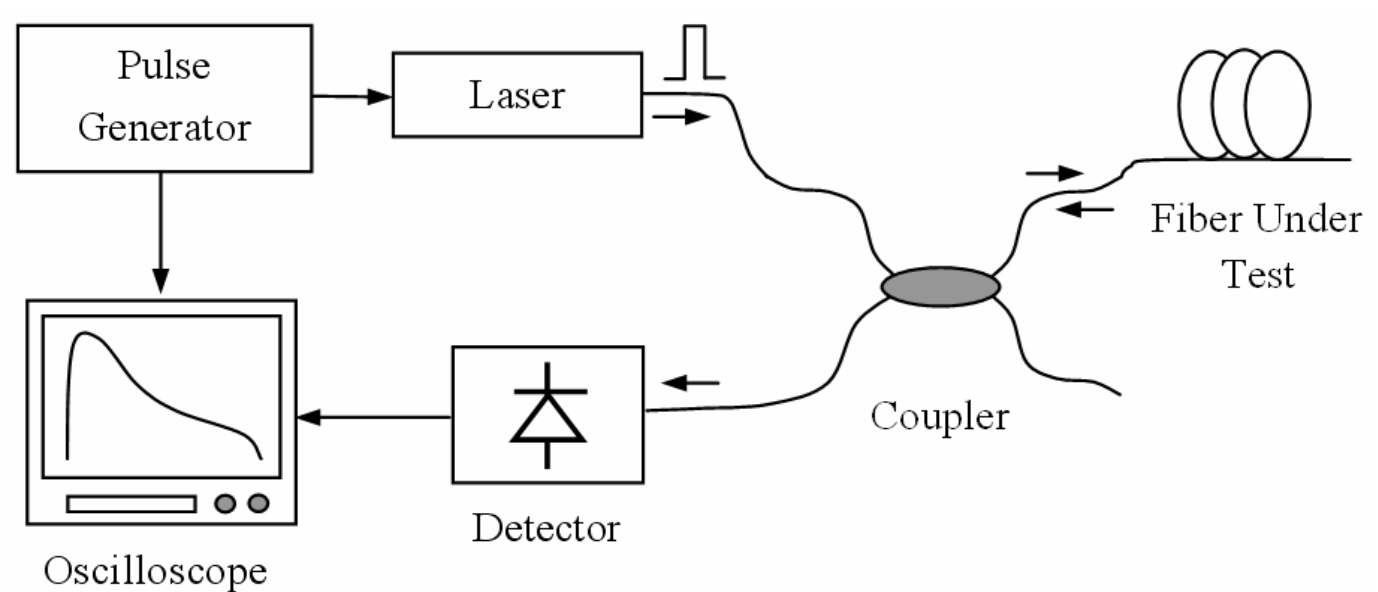

Figure 3. Conventional OTDR setup

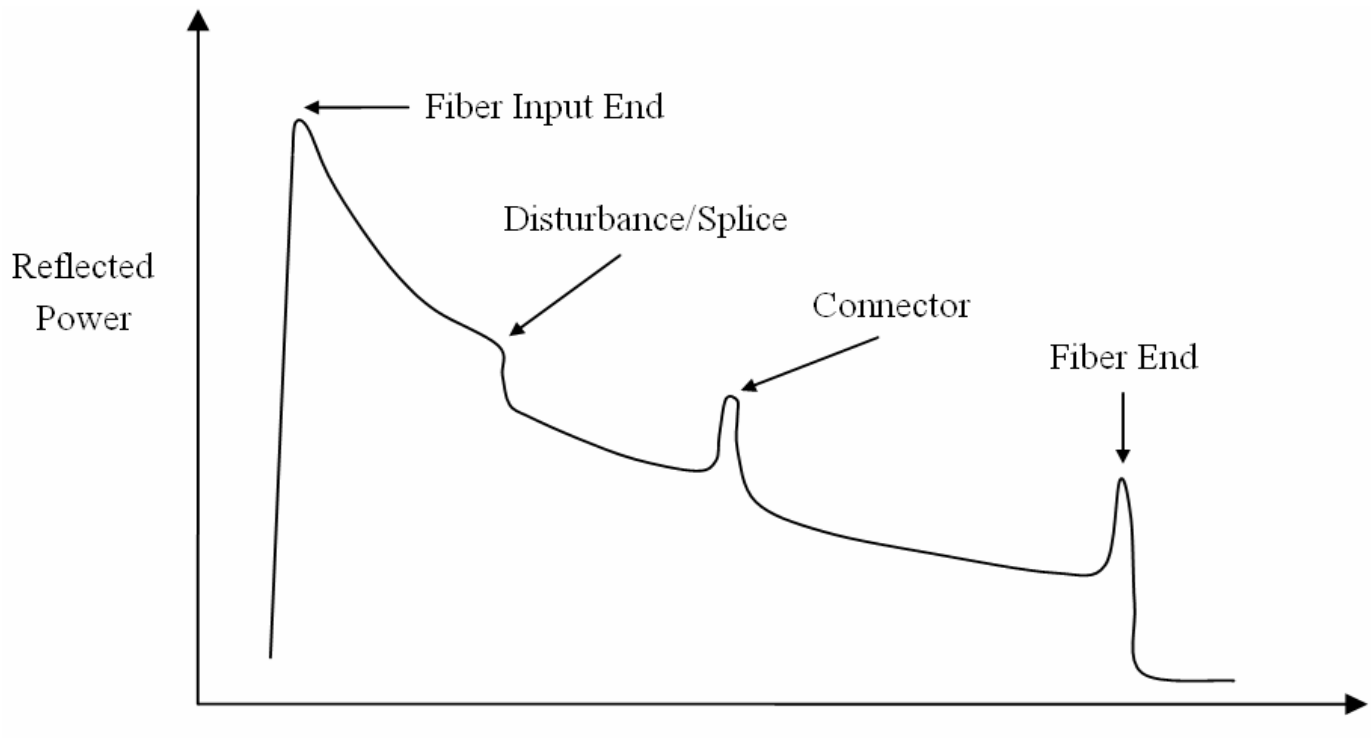

Time (Distance)

Figure 4. Conventional OTDR waveform 
The time delay, $\tau$, between the gated light pulse and the received optical power determines the distance along the fiber from the input end where the reflected light originated, such that

$$
\tau=\frac{2 L}{v_{g}}
$$

where $L$ is the distance to the point of reflection and $v_{g}$ the group velocity of the light in the fiber.

The spatial resolution, $\Delta z$, which is the minimum resolvable distance, is determined by the width of the launched pulse such that,

$$
\Delta z=\frac{T_{p} v_{g}}{2}
$$

where $T_{p}$ is the pulse width [17]

When the attenuation is constant for both directions of propagation, the backscattered power, $P_{s}$, from a distance, $L$, can be calculated as

$$
P_{s}=\frac{F \alpha_{s} T_{p} v_{g} P_{i} e^{-2 \alpha L}}{2}
$$

where $\alpha_{\mathrm{s}}$ is the Rayleigh scattering coefficient, $\alpha$ is the fiber loss coefficient, $F$ is the capture coefficient, and $P_{i}$ is the input power. 
Equations (2) and (3) illustrate the trade-off between spatial resolution and backscattered power. A narrower pulse improves the spatial resolution at the expense of a decreased SNR, which in turns limits the length of fiber that can be monitored.

\section{Phase-Sensitive OTDR}

A phase-sensitive OTDR ( $\phi$-OTDR) can serve as an intrusion sensor by enhancing coherent effects that have greater sensitivity to environmental perturbations than compared to intensity based sensors such as the conventional OTDR. A $\phi$-OTDR has the same setup as a conventional OTDR (Figure 3) except that the light source is a highly coherent laser. The output of such a sensor consists of the coherent interference of the backscattered fields from different points in the sensing fiber within the pulse width. One way to visualize this mechanism is shown in Figure 5, where backscattered light from a pulse of light interferes in a fashion similar to a Fabry-Perot interferometer. As illustrated in this example, at Time $=t_{0}$, an optical wave $\left(\mathrm{LE}_{\mathrm{M} 3}\right)$, will backscatter from the leading edge of the optical pulse at the location of some scattered referred to as Mirror 3. At Time $=\mathrm{t}_{\mathrm{o}}+T_{p} v_{g} / 4$, the pulse will have traveled half a pulse width downstream and another optical wave $\left(\mathrm{TE}_{\mathrm{M} 2}\right)$ will backscatter from another scatterer, Mirror 2. The two optical waves $\mathrm{LE}_{\mathrm{M} 3}$ and $\mathrm{TE}_{\mathrm{M} 2}$ will then interfere like they would in a Fabry-Perot interferometer as shown in Figure 6.

For the Fabry-Perot interferometer, the reflectance, $R_{F P}$, can be written as 


$$
R_{F P}=\frac{P_{r}}{P_{i}}=2 R(1+\cos \phi)
$$

with $P_{r}$ the reflected optical power, $P_{i}$ the incident optical power, and mirror reflectances $R=R_{1}=R_{2}<<1$. The round-trip optical phase shift, $\phi$, can be written as

$$
\phi=\frac{4 \pi n}{\lambda} L
$$

with $n$ the refractive index of the fiber, $\lambda$ the free-space wavelength and $L$ the cavity length.

Thus, when a certain point in the sensing fiber experiences a perturbation (such as an intruder stepping over the buried fiber), the refractive index and/or length of the fiber will change at that location producing a localized phase change in the optical wave. Backscattered light originating downstream from this point will then experience a roundtrip phase shift. As a result, the intensity of the interference will change at a time corresponding to the location of the perturbation as covered by Equation 1. If both the light source and sensing fiber are stabilized, the output interference pattern - the OTDR trace - should remain stable in time other than at the point of the perturbation. Hence, this change and therefore the perturbation, can be detected by subtracting the postperturbation $\phi$-OTDR trace from the pre-perturbation trace as shown in Figure 7. 

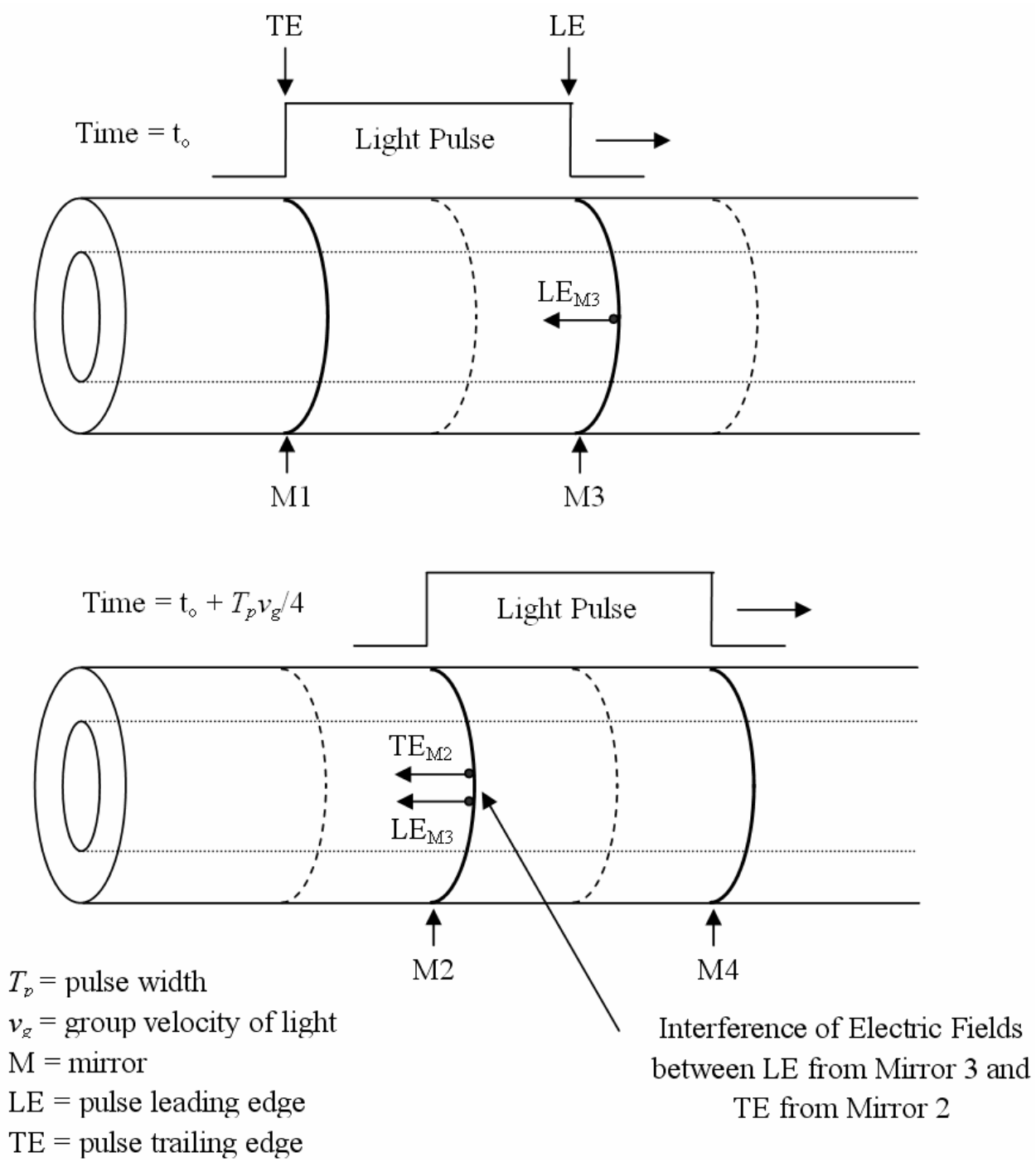

Figure 5. Interference of backscattered light arising from an optical pulse 


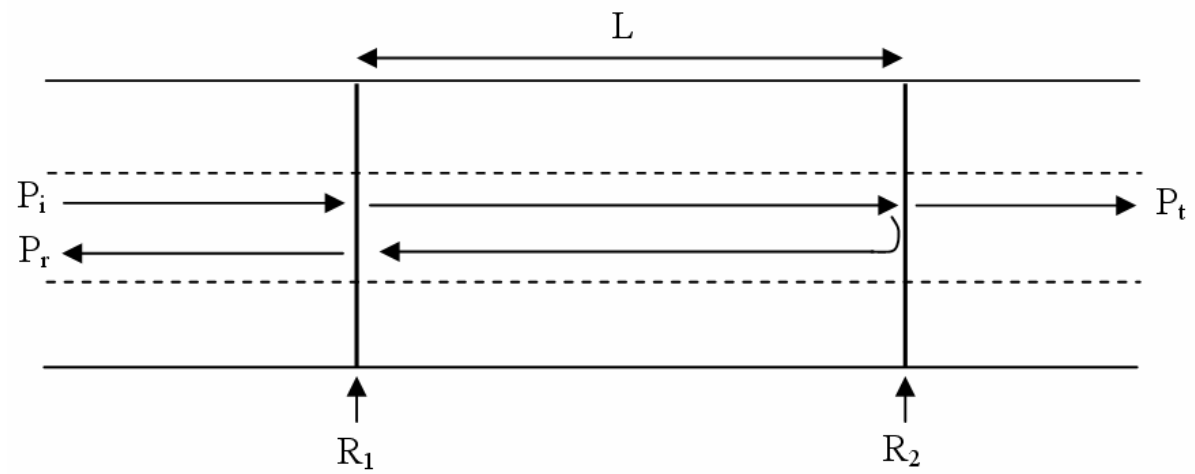

$\mathrm{R}_{1}, \mathrm{R}_{2}$ : reflectances

$\mathrm{L}$ : interferometer cavity length

$\mathrm{P}_{\mathrm{i}}$ : input power

$\mathrm{P}_{\mathrm{t}}$ : transmitted power

$\mathrm{P}_{\mathrm{r}}:$ reflected power

Figure 6. Fabry-Perot interferometer in an optical fiber

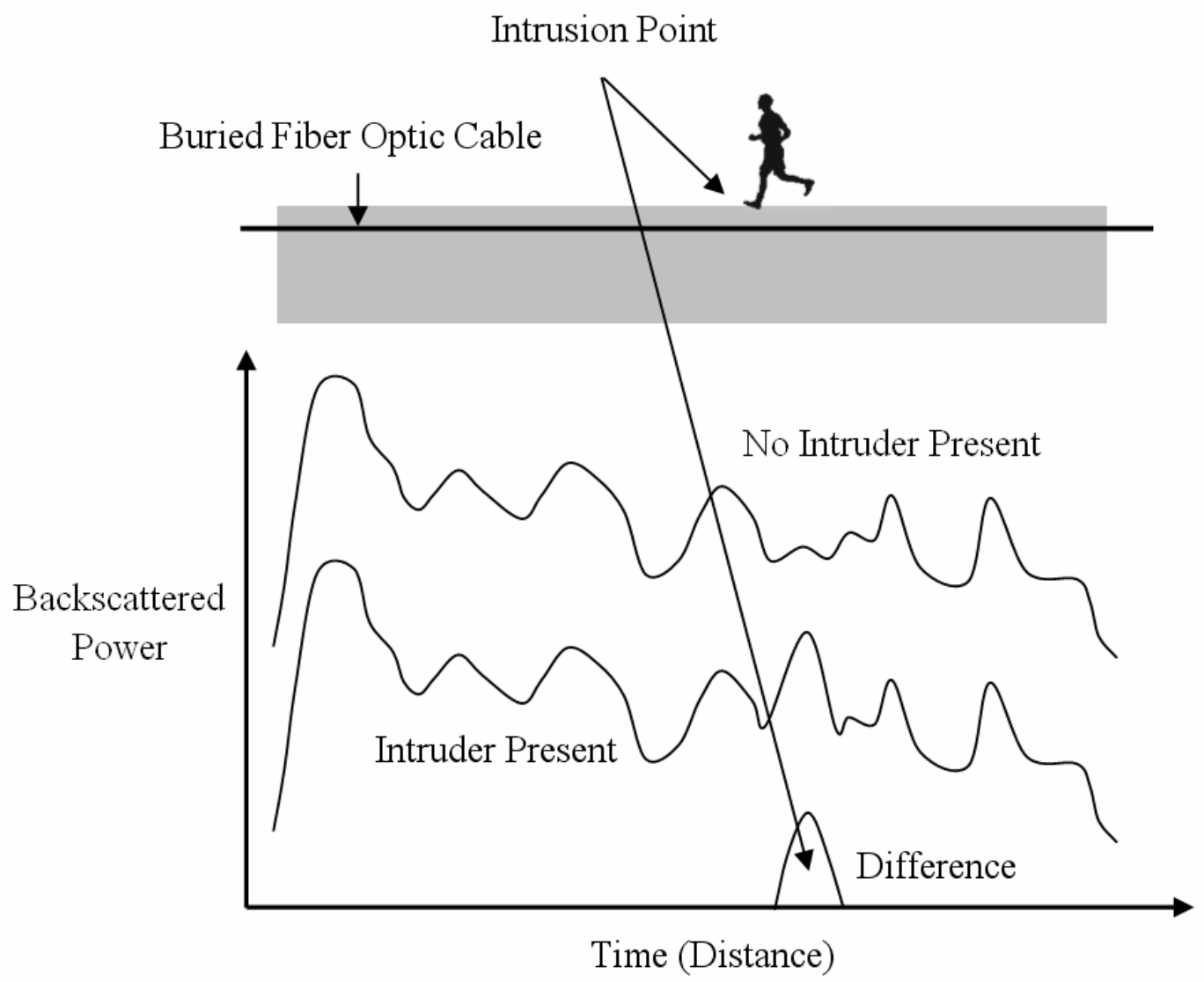

Figure 7. Phase-sensitive $\phi$-OTDR used as an intrusion sensor 


\section{Polarization of Rayleigh Backscattering}

The initial polarization state of light in single mode fiber is not typically maintained because of birefringence that varies randomly throughout the length of the fiber due to non-symmetrical core shape, anisotropic refractive index distribution in the core region, bending, twisting, and external pressure among other causes. When the optical wave is traveling in the z-direction in a lossless single-mode fiber, the electric field of the fundamental mode, $L P_{01}$, has two orthogonal polarizations, along the $\mathrm{x}$ - and y-axes, respectively as described by

$$
\begin{aligned}
& \vec{E}(z)=\left(\vec{E}_{x} e^{j \beta_{x} z}+\vec{E}_{y} e^{j \beta_{y} z}\right) e^{-j w t} \\
& \beta_{x}=2 \pi n_{x} / \lambda_{o} \text { and } \beta_{y}=2 \pi n_{y} / \lambda_{o}
\end{aligned}
$$

where $\vec{E}_{x}$ and $\vec{E}_{y}$ are complex values denoting the amplitude and phase of each polarization mode, $\beta_{x}$ and $\beta_{y}$ are the propagation constants of each polarization mode, $\lambda$ is the free-space wavelength of injected light, and $n_{x}$ and $n_{y}$ are the refractive indices of polarization modes in the optical fiber [6].

While in ideal fibers the initial polarization state will remain unchanged, in real fibers, the two polarization modes will propagate with different phase velocities resulting in a phase difference between the two modes after a certain length of fiber. Therefore, the polarization state will evolve continuously between linear, circular, and elliptical due to the birefringence of the fiber. 
While intensity based sensors such as the conventional OTDR are for the most part insensitive to polarization, a $\phi-\mathrm{OTDR}$ is highly sensitive to the polarization of the backscattered light because the visibility of the interference pattern within the optical pulse width is dependent upon the relative polarization between interfering fields. As stated earlier, this interference is similar to that occurring in a Fabry-Perot interferometer; In that case, however, the backscattered optical waves were assumed to have the same polarization yielding maximum visibility in the interferometer. In the opposite case, where the polarizations of the two optical waves are orthogonal to each other, the visibility will be zero, and any phase changes produced by an intruder will not be detected because of this "fading" effect. In cases where the relative difference between the polarizations of the optical waves are between $0^{\circ}$ and $90^{\circ}$, the visibility will be at an intermediate between the maximum and minimum of zero and determined by the dot product of the two [6].

\section{E. Pressure Response of a Buried Cable}

The sensitivity of a buried fiber optic cable to the pressure of an intruder crossing over it is a key issue in achieving a practical intrusion detection system. Even though it is possible to calculate the phase shift due to lateral pressure on a fiber, such calculations do not adequately account for the influence of the cable itself and the surrounding soil composition and conditions. Thus, experiments were undertaken at Texas A\&M University to measure the phase change produced by a weight on the ground above a buried fiber cable [22] 
First, a small (3-mm diameter) commercial cable containing a single mode fiber was buried at a known depth in a sand box and then spliced into one arm of an all-fiber Mach-Zehnder interferometer. A box with dimensions $30 \mathrm{~cm}$ x $10 \mathrm{~cm}$ (approximately the size of a human foot) was placed directly above the buried cable, and weights were added to the box to produce $\pi$-radian phase shifts as determined by monitoring the interferometer output power. For a depth of $20 \mathrm{~cm}$ it was found that a $60-\mathrm{kg}$ intruder produced a phase change of about $6 \pi \mathrm{rad}$, while for a $40 \mathrm{~cm}$ depth the expected phase change is about $2.5 \pi \mathrm{rad}$. Lastly, for a given burial depth the induced phase change was found to be close to a linear function of the applied weight.

Further investigations were made to determine if it were possible to avoid detection by stepping over the sensor cable. A $5 \mathrm{~m}$ long fiber cable was buried at a depth of $30 \mathrm{~cm}$ in clay soil and spliced into the Mach-Zehnder interferometer. It was found that a $60-\mathrm{kg}$ intruder produces a $\pi$-rad phase shift within approximately $2 \mathrm{~m}$ on either side of the buried cable, and several- $\pi$ rad phase shift when directly over the cable [5]. Additionally, it was found that seismic signals in the earth excited by an automobile driving down a road or by a runner could easily be sensed by the buried interferometer, even when the source of the disturbance was over $10 \mathrm{~m}$ away. In all the buried cable tests, the Mach-Zehnder interferometer signals were stable with time, indicating that the earth is an excellent thermal insulator and heat sink, serving as a low-pass filter for diurnal temperature changes. Thus these experiments indicate that a commercial fiber optic cable is suitable as the sensing element in a practical intrusion sensor system. 


\section{F. Simulation of $\phi$-OTDR Response to a Phase Perturbation}

In the $\phi$-OTDR, a light pulse of width $\tau$ is coupled into the fiber and the backscattered light is converted to an electrical signal of duration $T$, where $T=2 n_{g} L / c$, with $\mathrm{L}$ the fiber length, $\mathrm{n}_{\mathrm{g}}$ the group refractive index for the fiber mode, and $\mathrm{c}$ the freespace speed of light. For a silica fiber with $n_{g}=1.46$, it is calculated that $\mathrm{T}=9.73 \mathrm{~L}$, with $\mathrm{T}$ in $\mu$ s and $\mathrm{L}$ in $\mathrm{km}$. Thus, for a $20 \mathrm{~km}$ fiber, the duration of the return signal is $195 \mu$ s. A signal processor for analyzing the $\phi$-OTDR data will digitize the return signal at a sampling rate $1 / \mathrm{f} \tau$, with $\mathrm{f}$ a constant $<1$. Thus, if $\tau=2 \mu \mathrm{s}$ and $\mathrm{f}=0.1$, the sampling rate would be $5 \mathrm{MHz}$.

An analytical model used for predicting the $\phi$-OTDR performance assumes that the Rayleigh backscattering originates from a large number of centers with equal scattering cross-sections, randomly distributed at locations $\left\{z_{\mathrm{m}}\right\}$ along the fiber [6]. It is assumed that the light source is monochromatic at a wavelength of $1530 \mathrm{~nm}$ and that the modulator produces a square light pulse of width $\tau$. The average spacing, $\delta$, for the randomly positioned mirrors is taken to be $0.02 \mathrm{~m}$, much less than the spatial extent of the laser pulse in the fiber (e. g., a $2 \mu$ s pulse would have a spatial extent of $200 \mathrm{~m}$ ).

The Monte Carlo method was used to set the random locations of the scattering centers in the fiber $\left\{\mathrm{z}_{\mathrm{m}}\right\}$. A typical result showing Rayleigh-backscattered power vs. time for a $1 \mathrm{~km}$ length of fiber is given in Figure 8 . The pulse width is $0.5 \mu \mathrm{s}$, the sampling rate is $20 \mathrm{MHz}$, and the optical power entering the fiber during the pulse is 50 $\mathrm{mW}$. Shot noise calculated using the Monte Carlo method is superimposed on each 
sample of the Rayleigh-backscattered signal. Conditions are the same for signal records $\mathrm{S} 1$ and $\mathrm{S} 2$, except that a $\pi / 4$ phase change was applied to the fiber at a distance of $500 \mathrm{~m}$ from the launch point prior to generating S2.

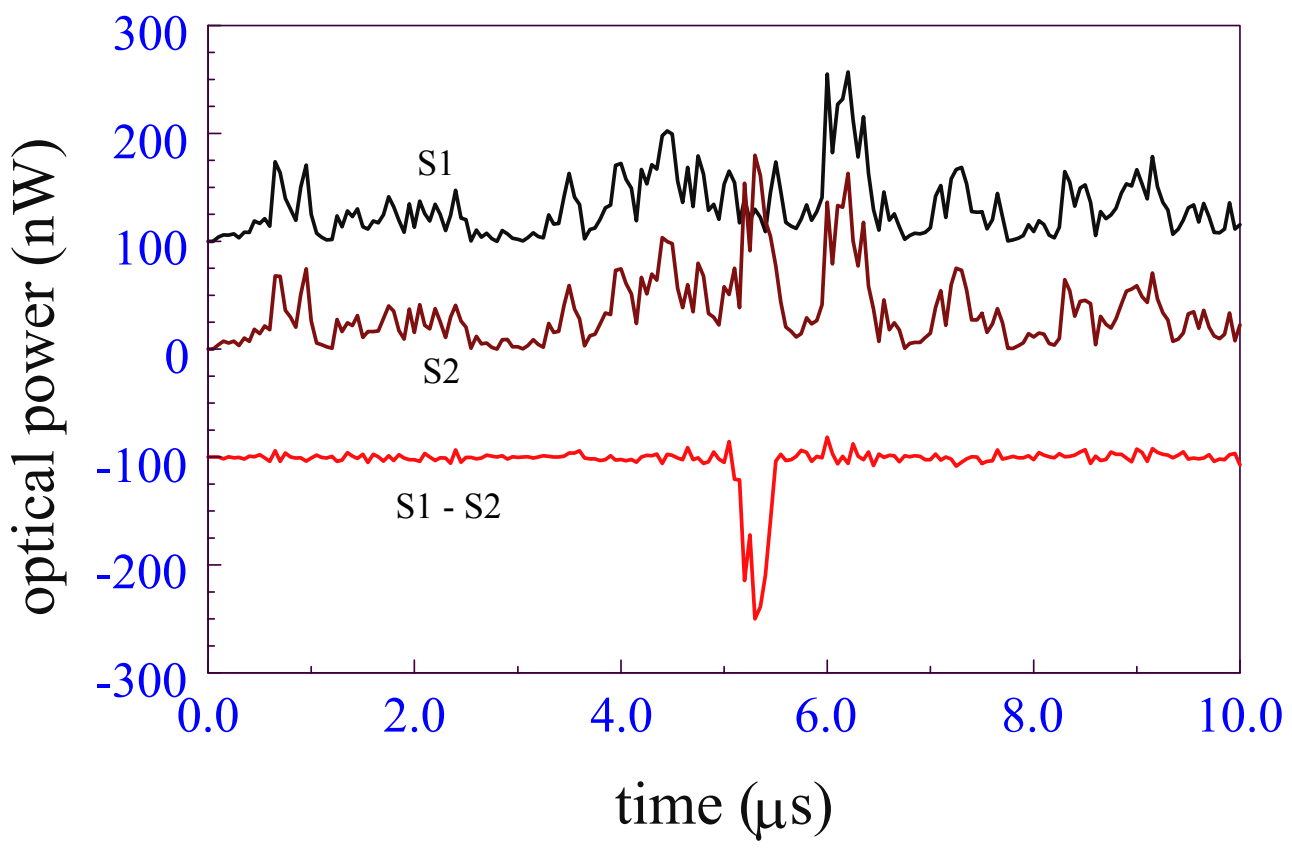

Figure 8. Simulation result showing effect of a localized $\pi / 4$ phase perturbation on the temporal dependence of Rayleigh backscattered power. The upper and lower traces have been vertically displaced by $\pm 100 \mathrm{nW}$ so that the curves can easily be distinguished from one another.

If the perturbation-induced change in the averaged signal in bin $\mathrm{j}^{*}$ did not exceed the threshold, then a missed intruder was indicated; while a false alarm corresponded to the case that the change exceeded the threshold in a time bin in which no perturbation was applied. Threshold levels $\left\{\varepsilon_{\mathrm{j}}\right\}$ for the time bins were chosen to provide low false alarm probability and low missed intruder probability. The number of missed intruders in a total of 100,000 trials was determined for time bins at different ranges. In this 
manner, the range consistent with a particular range resolution and missed intruder probability were determined.

Calculated system performance is shown in Figure 9 for an assumed missed intruder probability of $10^{-4}$ and a false alarm rate of $10^{-10}$. The range resolution was varied by changing the pulse width $\tau$. Ten-pulse averages were used in this calculation.

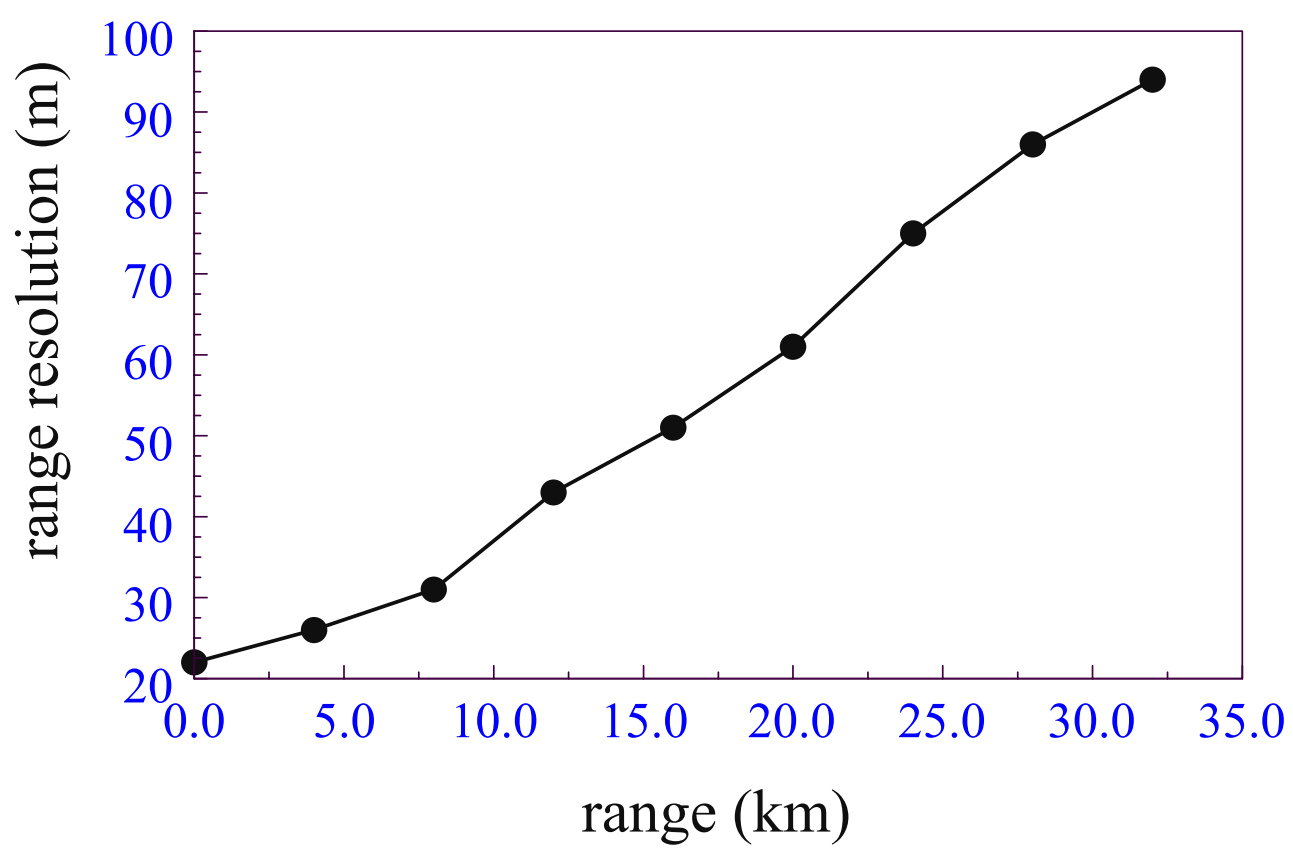

Figure 9. Simulation result for dependence of range resolution on range in the fiber.

\section{G. Frequency Drift Measurement}

To characterize the frequency drift of the laser, an all-fiber Mach-Zehnder interferometers (MZI) can be used. The MZI is designed to be unbalanced so that one 
path length is longer than the other (Figure 10), so that a relative phase difference $\phi_{1}-\phi_{2}$ in the interferometer is created and is given by

$$
\phi_{1}-\phi_{2}=\frac{2 \pi n \Delta v}{c}\left(L_{1}-L_{2}\right)
$$

with $\mathrm{n}$ the effective refractive index fiber mode, $\Delta v$ the laser frequency drift, $\mathrm{L}_{1}$ and $\mathrm{L}_{2}$ the lengths of the fiber arms, and $\mathrm{c}$ the free-space speed of light. If the laser frequency drift is linear with time, the relative phase shift can be written

$$
\phi_{1}-\phi_{2}=\frac{2 \pi n}{c}\left(L_{1}-L_{2}\right)\left(v_{o}+\alpha t\right)
$$

with $\alpha$ the frequency drift rate. The photodetector signal current, I, can be written

$$
\mathrm{I}=\mathrm{I}_{0}\left[\mathrm{C}_{1}+\left(\mathrm{C}_{2} \cos \phi_{1}-\phi_{2}\right)\right]
$$

with $\mathrm{I}_{0}, \mathrm{C}_{1}$, and $\mathrm{C}_{2}$ constants. If the laser frequency drift is linear in time as in (9), the photodetector signal will vary sinusoidally, sweeping through interference "fringes" at a rate proportional to the frequency drift rate. The amount of frequency drift corresponding to one fringe is determined by the path length difference. For example, if $\mathrm{L}_{1}-\mathrm{L}_{2}=200 \mathrm{~m}$, then one fringe ( $2 \pi$ radian phase shift) will correspond to a $1 \mathrm{MHz}$ frequency drift in the laser.

Once the MZI is stabilized, it is possible to monitor the frequency drift of the laser; however, the drift rate recorded by the photodetector represents the combined effects of 
the laser and of the Mach-Zehnder. By utilizing two Mach-Zehnders and verifying similar readings, an accurate drift rate can be ascertained. The interferometer data can also be useful in assessing "mode hopping" (sudden frequency shifts) in the laser, which result in discontinuities in the fringe patterns.

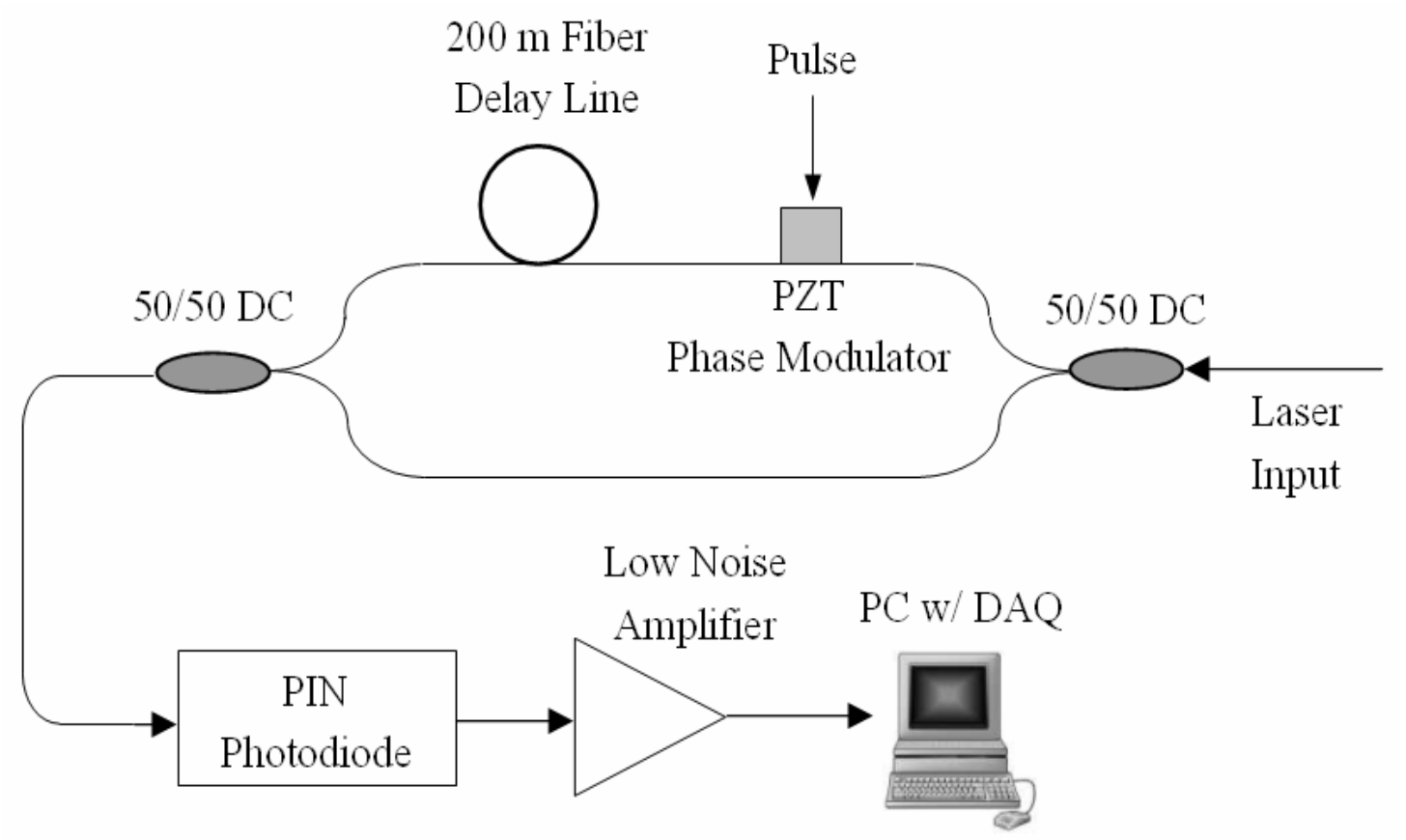

Figure 10. Mach-Zehnder test setup for frequency drift measurements. 


\section{CHAPTER III}

\section{ERBIUM DOPED FIBER LASER CHARACTERIZATION}

\section{A. Er:Fiber Laser}

For a practical intrusion sensor system based on the $\phi$-OTDR, a laser with minimal frequency drift and a narrow instantaneous linewidth is required. In previous work at Texas A\&M University, a narrow linewidth, low frequency drift rate Erbiumdoped fiber laser (Figure 11) was developed [8] and applied to the first laboratory demonstration of the $\phi$-OTDR [16]. Substantial improvements, however, in the frequency drift rate and sensitivity to temperature and acoustic effects were required before field tests could be carried out.

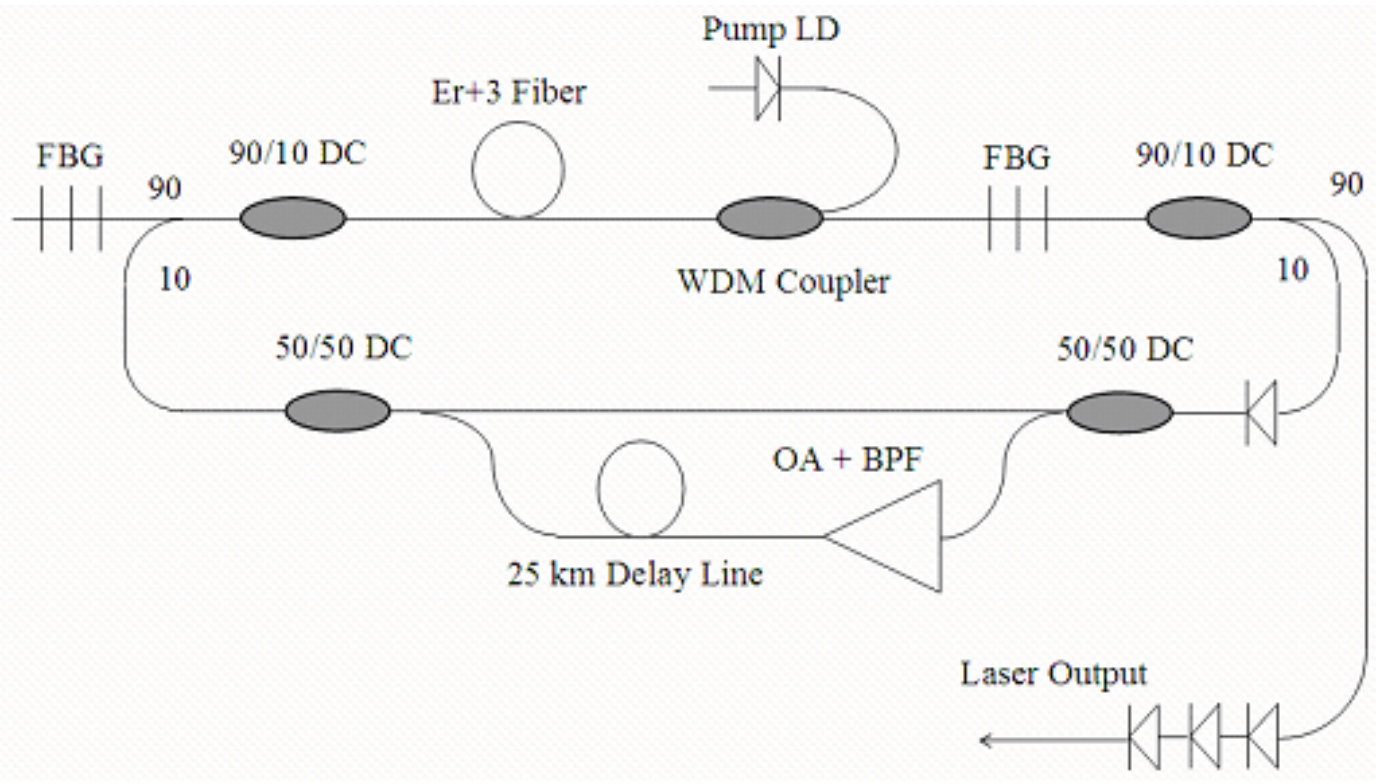

Figure 11. Previously researched fiber laser setup with short and long optical feedback paths 
The experimental setup for the initial system light source in this research is shown in Figure 12 and is a modified version from that shown in Figure 11. This improved light source also utilizes all single mode fiber paths and is based on a FabryPerot cavity that is formed by two fiber Bragg grating (FBG) reflectors with identical reflectance peak wavelengths of $1555.4 \mathrm{~nm}$ and spectral widths of $0.4 \mathrm{~nm}$. The FBG reflectances are $99.9 \%$ (back side) and 92\% (output side). The $3 \mathrm{~m}$ long Er+3 doped fiber gain medium $(7 \mathrm{~dB} / \mathrm{m}$ gain) is pumped by a $980 \mathrm{~nm}$ semiconductor pump laser diode (LD) via a wavelength division multiplexing (WDM) coupler. A short ( 1 m) optical feedback loop coupled to the laser cavity via two 90/10 directional couplers (DCs) was used to improve the spectral characteristics of the laser [8]. The long feedback loop from the laser in Figure 11, however, was removed due to instabilities arising in the laser frequency because of the propensity of the $25 \mathrm{~km}$ delay line to be easily influenced by environmental disturbances due to its long length. Additionally, the optical isolators at the output of the original laser were moved to the immediate output of the laser cavity to better suppress coupling of the laser emission back into the cavity for stable operation, but still ensure unidirectional propagation in the feedback loop.

Similar to the previous laser, the new laser is housed in a thermally and acoustically insulated enclosure, as a constant temperature environment and minimal acoustic disturbances are essential to achieving a stable single mode lasing spectrum with low frequency drift. To eliminate internal heat generation the pump laser and its power supply are located outside of the insulated enclosure containing the optical components of the laser. The optical output power from this laser is about $50 \mu \mathrm{W}$, and 
the emission wavelength of $1555.4 \mathrm{~nm}$ measured with an optical spectrum analyzer as shown in Figure 13 corresponds to the reflectance peak of the FBGs.

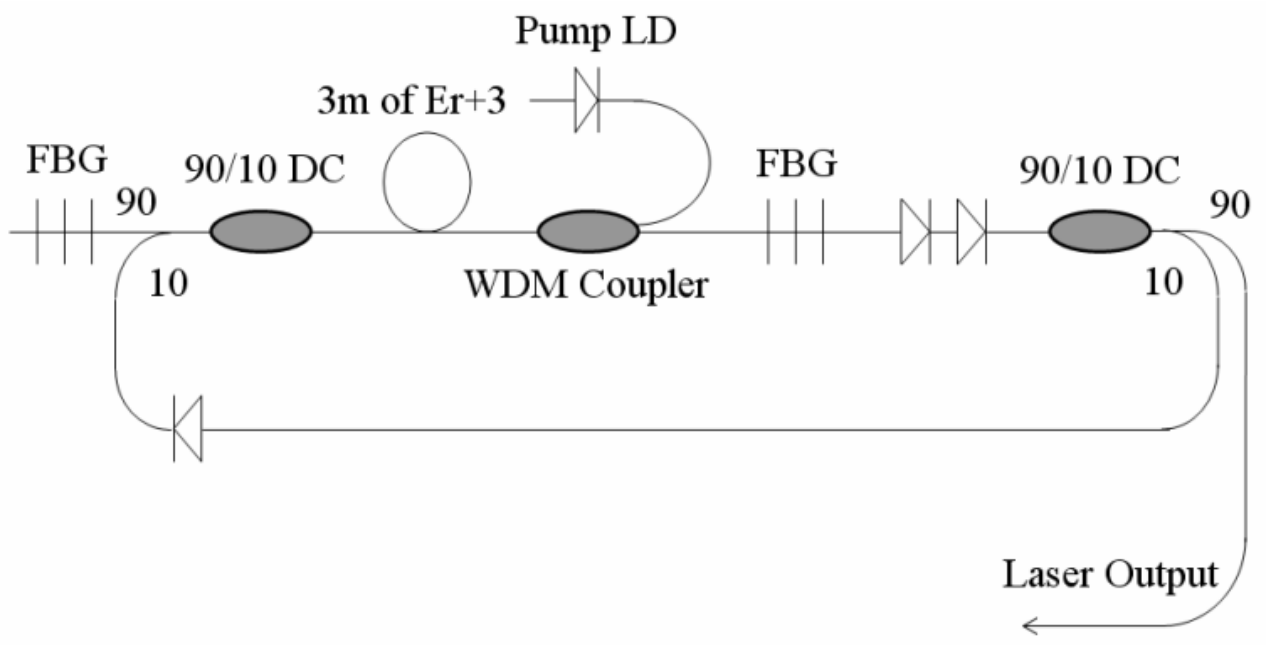

Figure 12. Experimental setup for fiber laser used in the intrusion sensor system

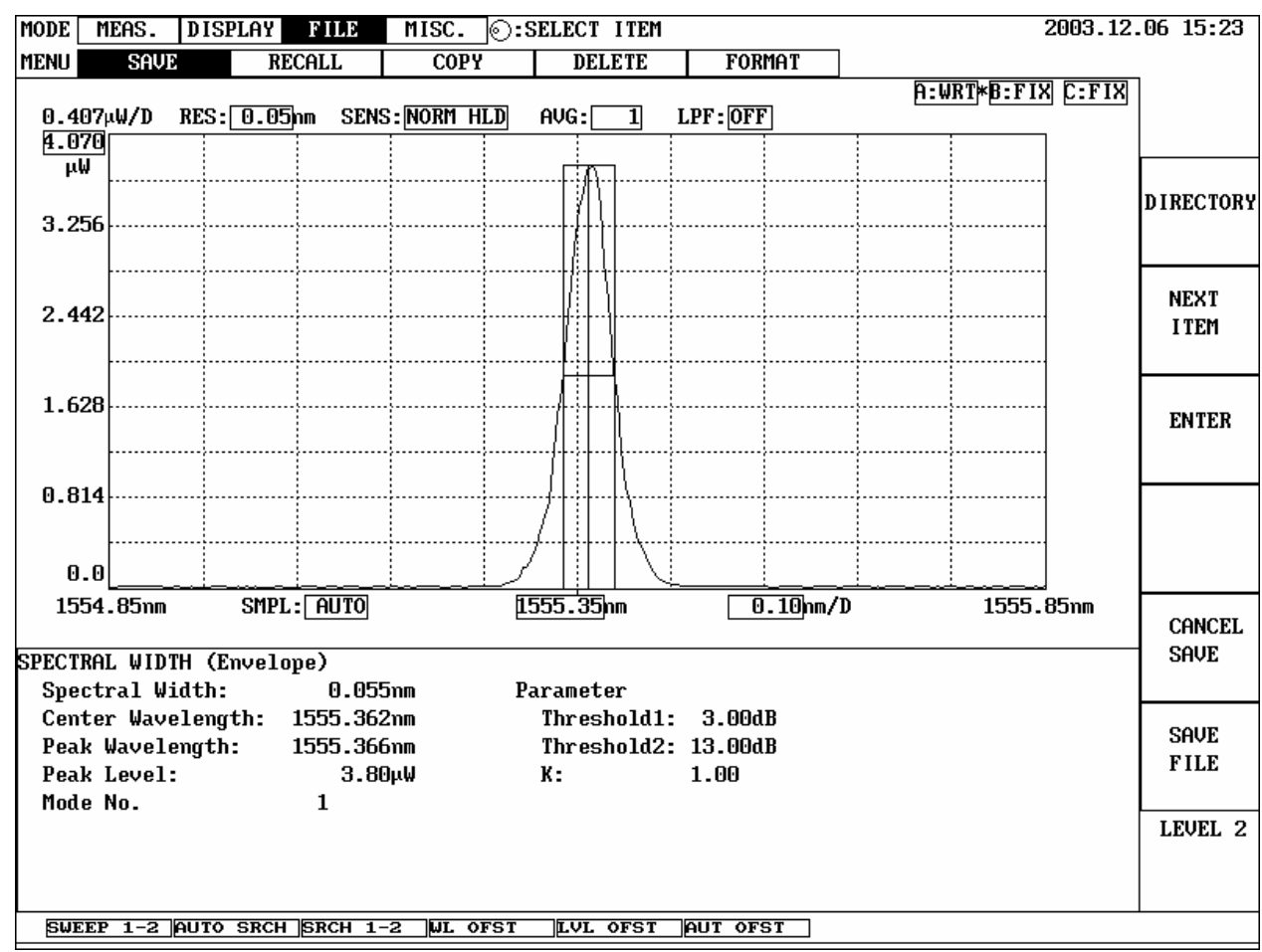

Figure 13. Spectral linewidth scan of Erbium doped fiber laser 


\section{B. Linewidth Measurements}

Conventional optical spectrum analyzers do not have the high resolution necessary to measure the narrow linewidth of a single longitudinal mode laser such as the one described in the previous section. Hence, a delayed self-heterodyne setup consisting of a fiber Mach-Zehnder interferometer with a $63 \mathrm{~km}$ delay line in one arm was used for the instantaneous linewidth measurement of the laser, as shown in Figure $14[23,24]$. An electrooptic modulator driven by a sinusoidal voltage shifts the center frequency of the correlated signal to $100 \mathrm{kHz}$. As shown in Figure 15, the laser exhibits a resolution-limited spectral width of $\leq 3 \mathrm{kHz}$. Such narrow spectral widths, indicative of single longitudinal mode operation, are frequently seen in these Er-fiber lasers [25-27]. The selection of a single longitudinal mode probably results from the formation of an intracavity refractive index grating due to spatial hole burning in the Er-doped fiber.

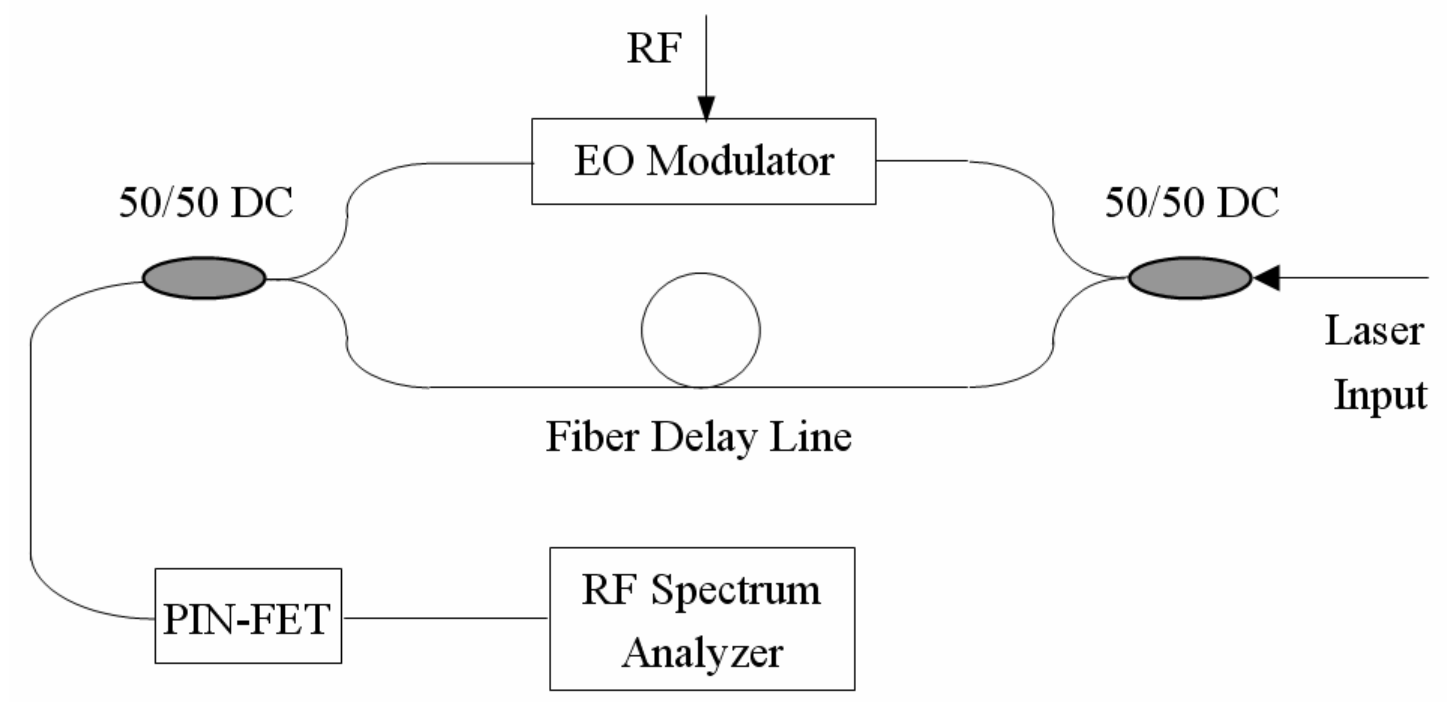

Figure 14. Delayed self-heterodyne test setup for instantaneous linewidth measurements 


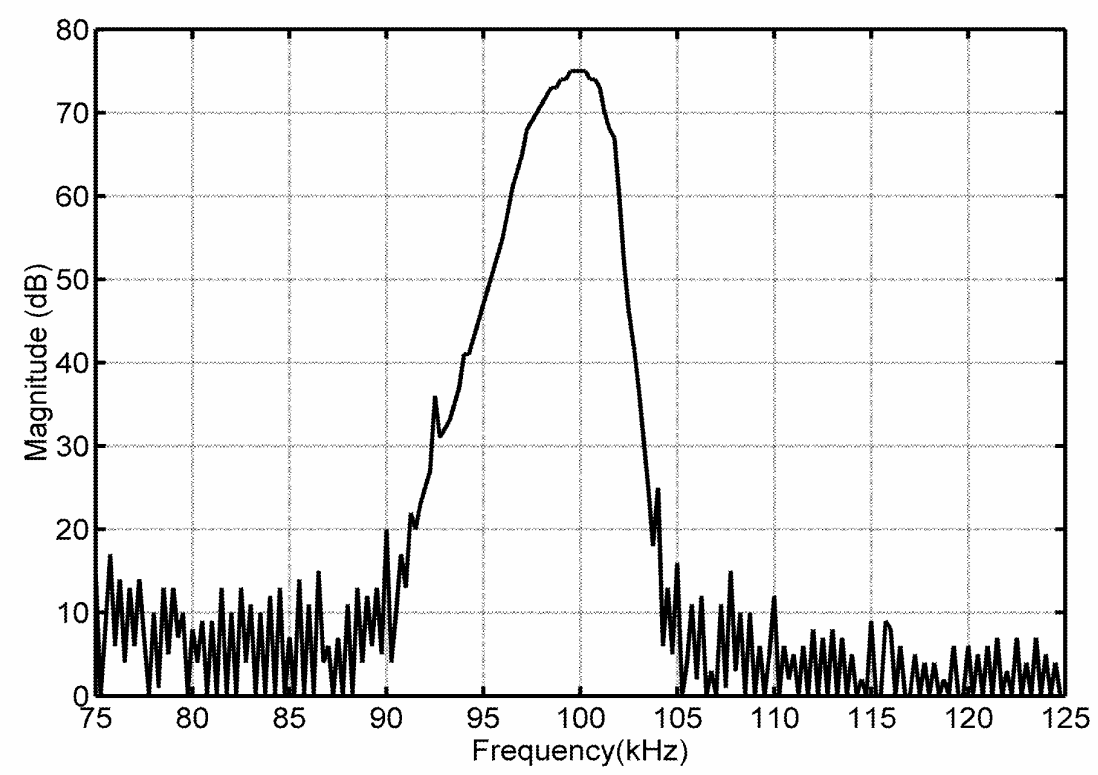

Figure 15. Delayed self-heterodyne measurement of laser linewidth

\section{Frequency Drift Measurements}

The rate of frequency drift was determined by observing temporal fringes in a pair of unbalanced fiber Mach-Zehnder interferometers (MZIs) with path length differences of $200 \mathrm{~m}$, as illustrated in Figure 16. From Equation 9, the $200 \mathrm{~m}$ path length difference dictates that an observed fringe ( $2 \pi$ radian phase shift) corresponds to a 1 $\mathrm{MHz}$ frequency change in the laser.

Both MZIs were insulated from thermal and acoustic effects with multiple layers of foam board and shredded Styrofoam. Although well-insulated, the two MZIs are themselves still affected to some extent by environmental perturbations. Having both MZIs individually packaged and physically separated provides for simultaneous monitoring of the laser to reduce the uncertainty of the origin of the observed fringes. 


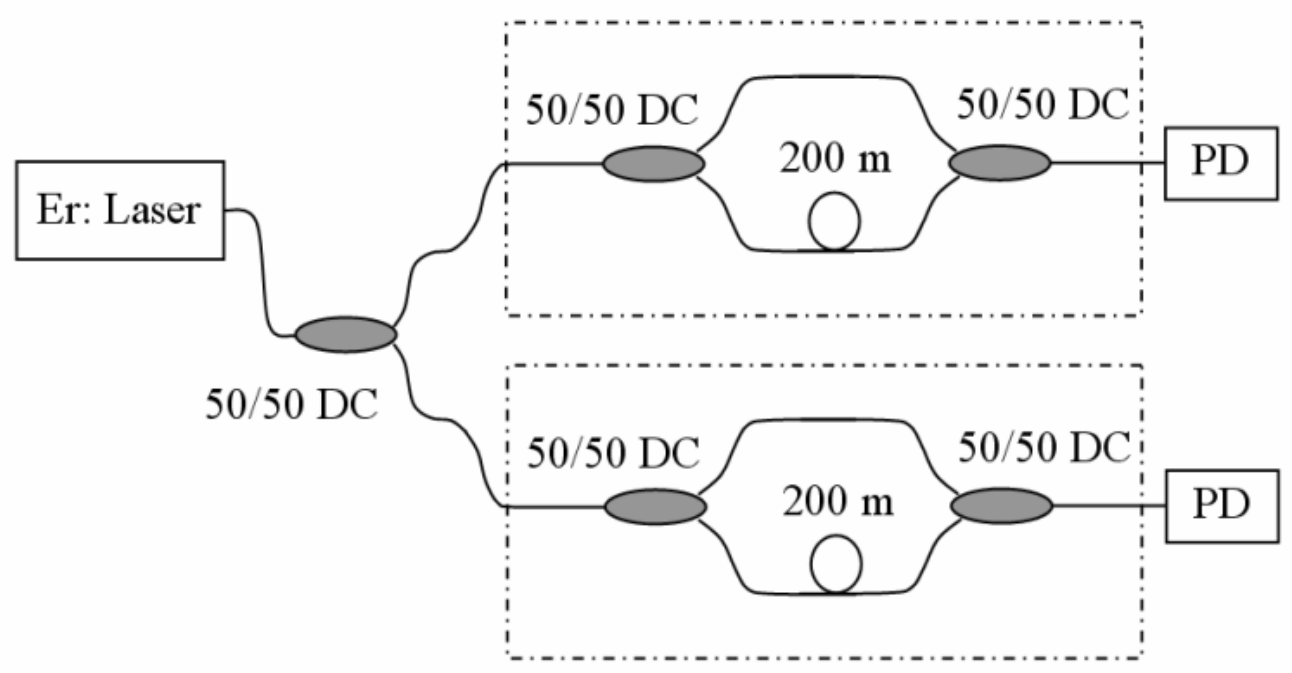

Figure 16. Laser frequency drift monitoring setup incorporating two Mach-Zehnder interferometers

As shown in Figure 17, insulating the MZIs significantly reduced environmental effects from affecting the frequency drift measurements. This is inferred because the fringes have a constant phase relative to each other, thus implying that the frequency drift measurements are due to the laser. When the ambient temperature change was relatively rapid, a frequency drift of $\sim 4-5 \mathrm{MHz} / \mathrm{min}$ was observed [Figure 17(a)]. Under normal laboratory conditions with minimal disturbances, frequency drifts of $\sim 1-1.5$ $\mathrm{MHz} / \min$ [Figure 17(b)] were routinely measured. Under the quietest of conditions, frequency drifts in the order of 100-300 kHz/min were observed [Figure 17(c)]. The MZIs were also stable enough to detect when the laser hopped modes as seen by the momentary phase shifts in Figure 17(a) and (c), which were captured simultaneously by both MZIs. These results show that the modifications to the laser and the added insulation reduced the frequency drift and its sensitivity to environmental effects enough for adequate use in a $\phi$-OTDR system. 


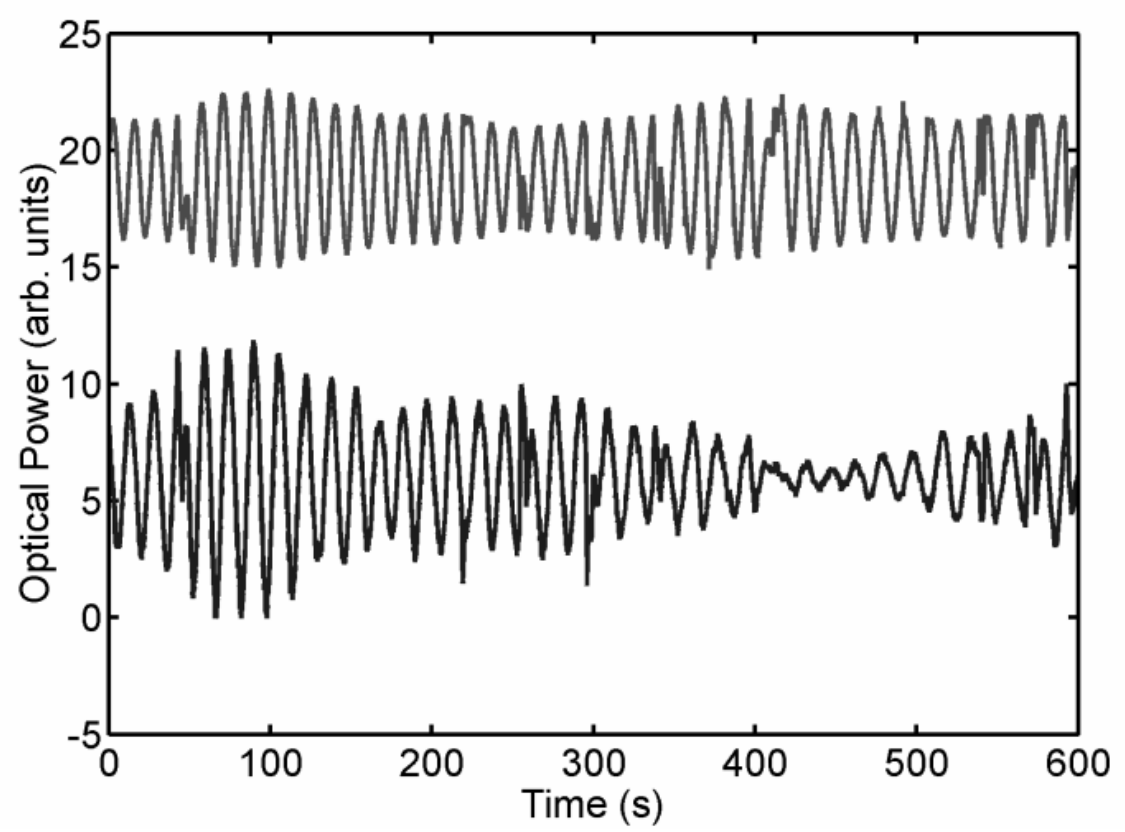

(a)

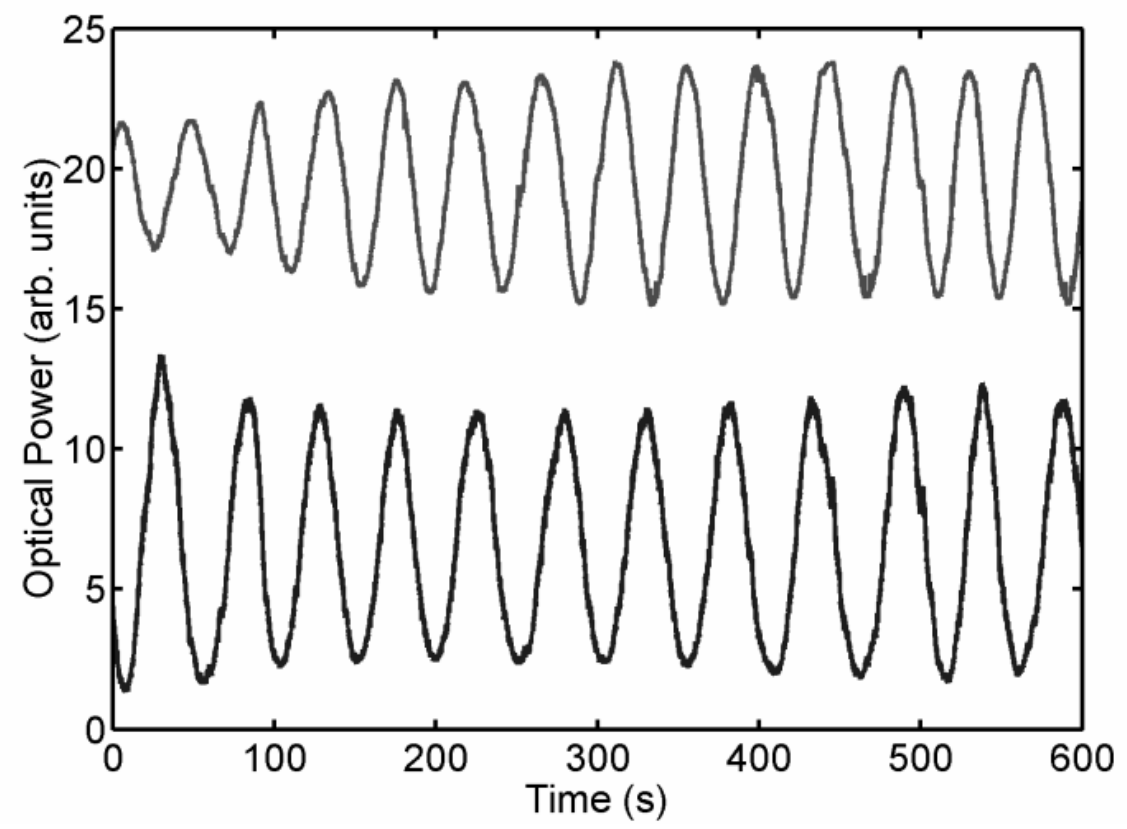

(b)

Figure 17. Laser frequency drift with: (a) environmental disturbances at 2:52:03 PM. (b) normal laboratory conditions at 8:36:44 AM. (c) minimal disturbances at 10:32:04 PM 


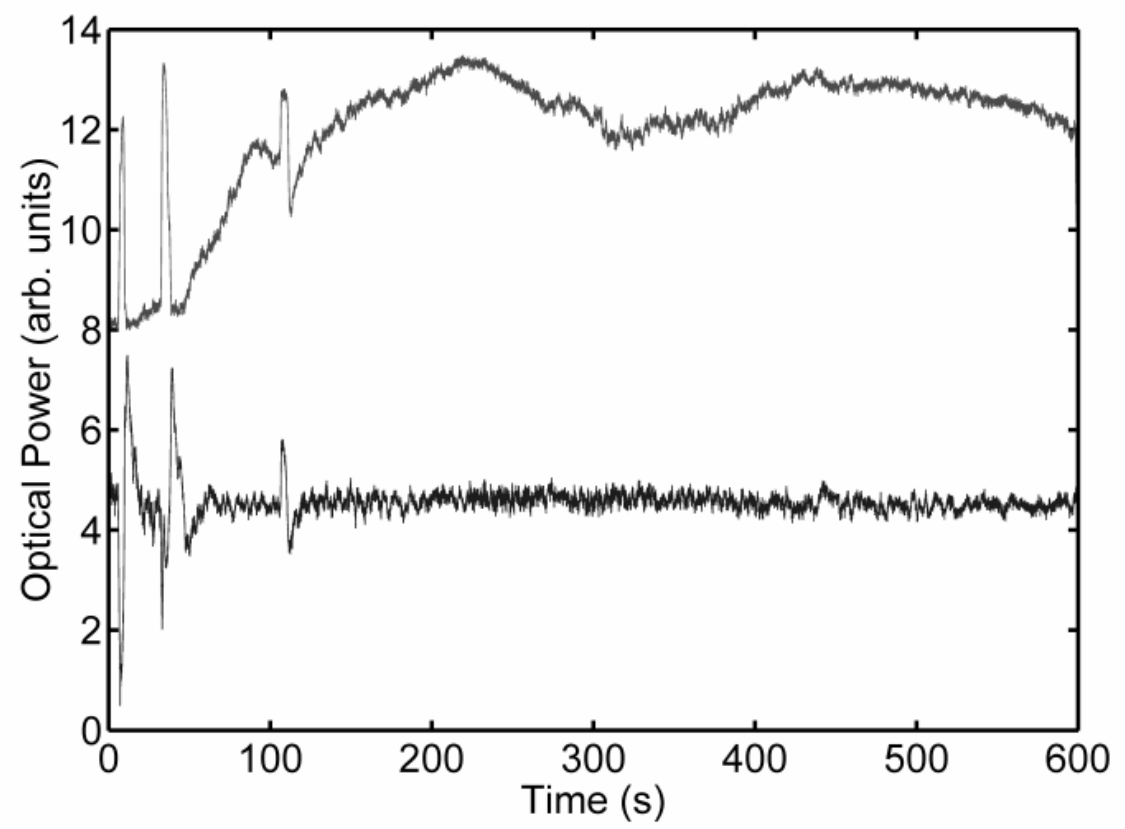

(c)

Figure 17. Continued 


\section{CHAPTER IV}

\section{OTDR LABORATORY EXPERIMENTS}

\section{A. Test Configuration}

Once the laser frequency stability was verified, the performance of the $\phi$-OTDR was investigated in a laboratory setting using the arrangement of Figure 18. Light from the $\mathrm{cw}$ laser shown in Figure 12 passed through a bandpass filter (BPF), consisting of a FBG with reflectance peak matched to the lasing wavelength in series with a circulator, to remove spontaneous emission. The light was then amplified by $\sim 16 \mathrm{~dB}$ with an erbium-doped fiber amplifier (EDFA), the output of which was filtered by a second BPF identical to the first. Narrow $(10 \mu \mathrm{s})$ light pulses from the laser were gated into the fiber with an electrooptic modulator (EOM), amplified by $\sim 30 \mathrm{~dB}$ with another EDFA, and coupled into the sensing fiber via a $3-\mathrm{dB}$ fiber optic directional coupler (50/50 DC).

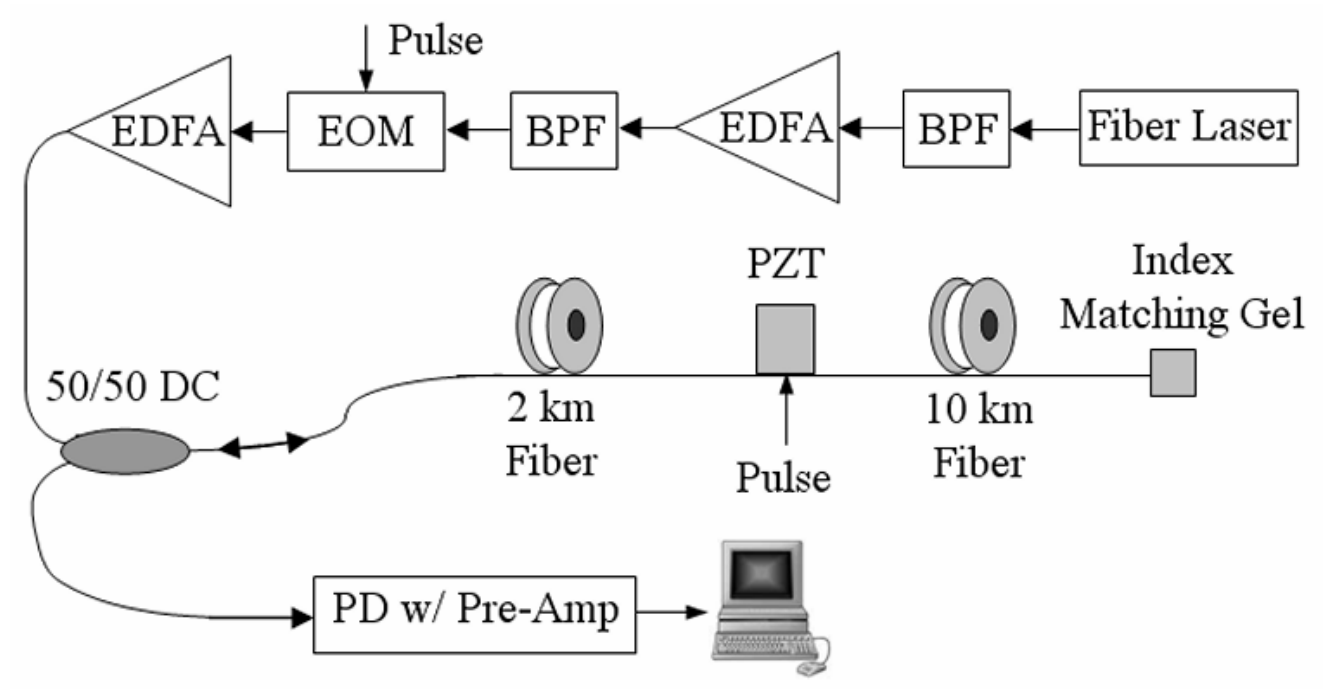

Figure 18. Laboratory setup for characterizing the $\phi$-OTDR system 
The distributed intrusion sensor was simulated by two thermally insulated spools of bare single mode fiber ( 2 and $10 \mathrm{~km}$ ) with a phase modulator (PZT), consisting of about $10 \mathrm{~m}$ of fiber wound on a piezoelectric fiber cylinder, spliced in between them to produce controlled phase changes simulating an intruder. The Rayleigh backscattered light from the sensing fiber passed through the 3-dB DC to an optical receiver containing an InGaAs photodiode and a transimpedence amplifier operating with a $40 \mathrm{~dB}$ gain and a $50 \mathrm{kHz}$ bandwidth. Data was acquired with a Tektronix 11201A Digitizing Oscilloscope sampling at $1 \mathrm{MS} / \mathrm{s}(\mathrm{MSamples} / \mathrm{s})$ and then transferred serially to the PC where it was processed with a LabView system.

\section{B. Test Results}

An initial test of the $\phi$-OTDR was designed to minimize the possibility of laser drift fluctuations from masking the effects of the PZT by testing if phase changes occurring between consecutive laser pulses (i.e. the minimum possible time between sensor interrogations) were detectable. To accomplish this, so that successive returns did not overlap, the EOM was pulsed continuously with a period of $150 \mu$ s, which exceeded the $120 \mu$ s round trip time for light propagation in the fiber, plus an additional $20 \mu$ s to account for time when the pulse is entering and leaving the fiber. The PZT element was then pulsed at a voltage and period that would induce a phase change of approximately $\pi / 2$ radians in every other $\phi$-OTDR trace (i.e. with a period of $300 \mu$ s). This test scheme is illustrated in Figure 19 where three EOM pulses and $\phi$-OTDR traces are visible along with the square pulse applied to the PZT during the middle $\phi$-OTDR trace. With this 
pulse configuration, phase changes are aimed to be produced in consecutive $\phi$-OTDR traces at the PZT location with minimal changes in the remainder of the trace.

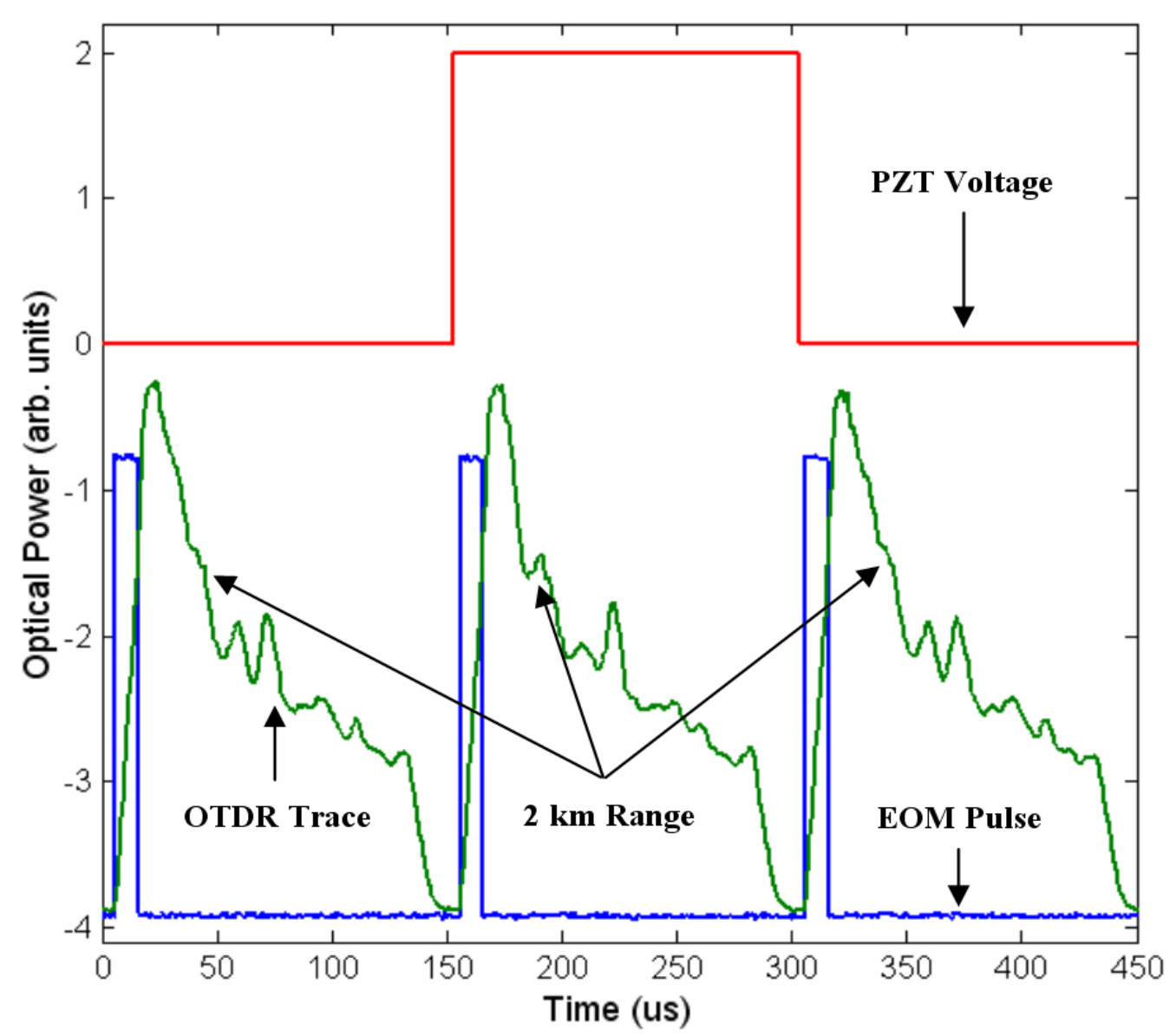

Figure 19. Three $\phi$-OTDR traces and pulse timing

Figure 20 displays two consecutive superimposed traces (one with the PZT applying a phase shift and one without) and the difference between the two traces that more clearly shows how pulsing the PZT produced a detectable phase change. This is seen in the difference waveform where an intensity change (due to the phase change) occurs $20 \mu \mathrm{s}$ from the start of the $\phi$-OTDR trace, corresponding to the round trip transit 
time for the 2-km range location of the PZT.

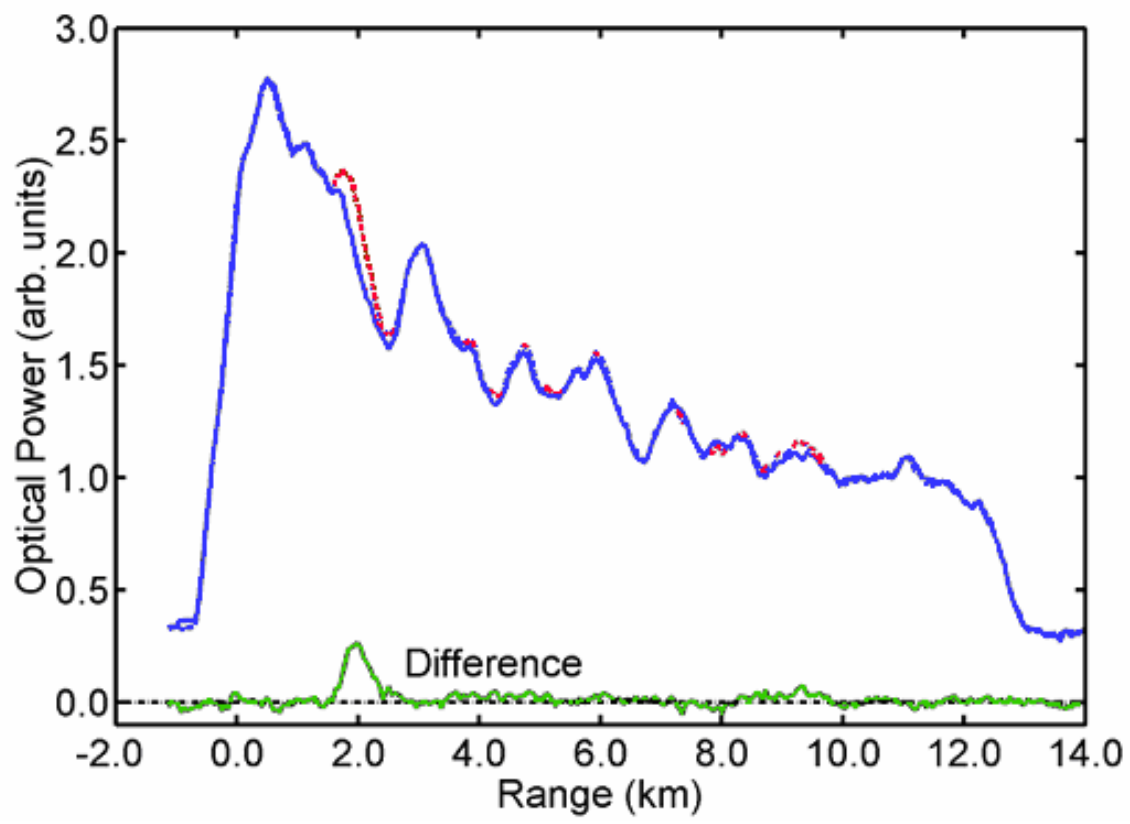

Figure 20. Effect of a phase perturbation on successive $\phi$-OTDR traces

In another test of the phase response of the system, ramp voltages varying through $>2 \pi$ radians were applied to the PZT element, and the amplitude changes over 64 consecutive $\phi$-OTDR traces were monitored. Superimposing the traces shows how the phase modulation causes a large change in the envelope at the $2 \mathrm{~km}$ location, while the remainder of the trace remains relatively constant [Figure 21(a)]. Subtracting these OTDR traces from a reference trace with no applied PZT voltage shows the varying intensities more clearly at the $2 \mathrm{~km}$ location [Figure 21(b)] with a spatial resolution of about $1 \mathrm{~km}$ due to the $10 \mu \mathrm{s}$ pulses of light (Equation 2) launched into the fiber. Finally, the dependence of the peak amplitude of the difference in these two traces is close to a 
sinusoidal function of applied phase shift [Figure 21(c)], which is expected because of the interferometric nature of the $\phi$-OTDR response.

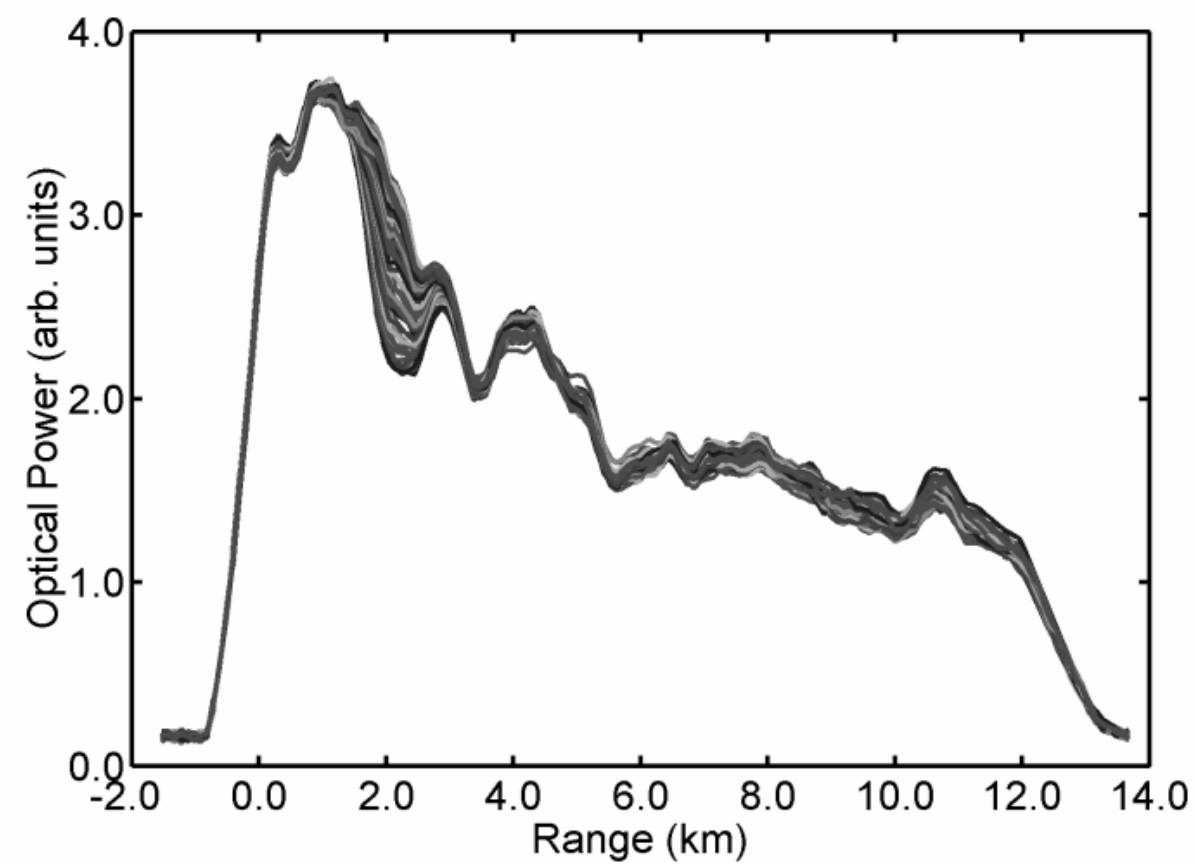

(a)

Figure 21. (a) Consecutive OTDR traces superimposed with phase changes at $2 \mathrm{~km}$ location. (b) Differences of OTDR traces superimposed with phase changes at $2 \mathrm{~km}$ location. (c) Magnitudes of $2 \mathrm{~km}$ location plotted vs. applied phase shifter voltage. 


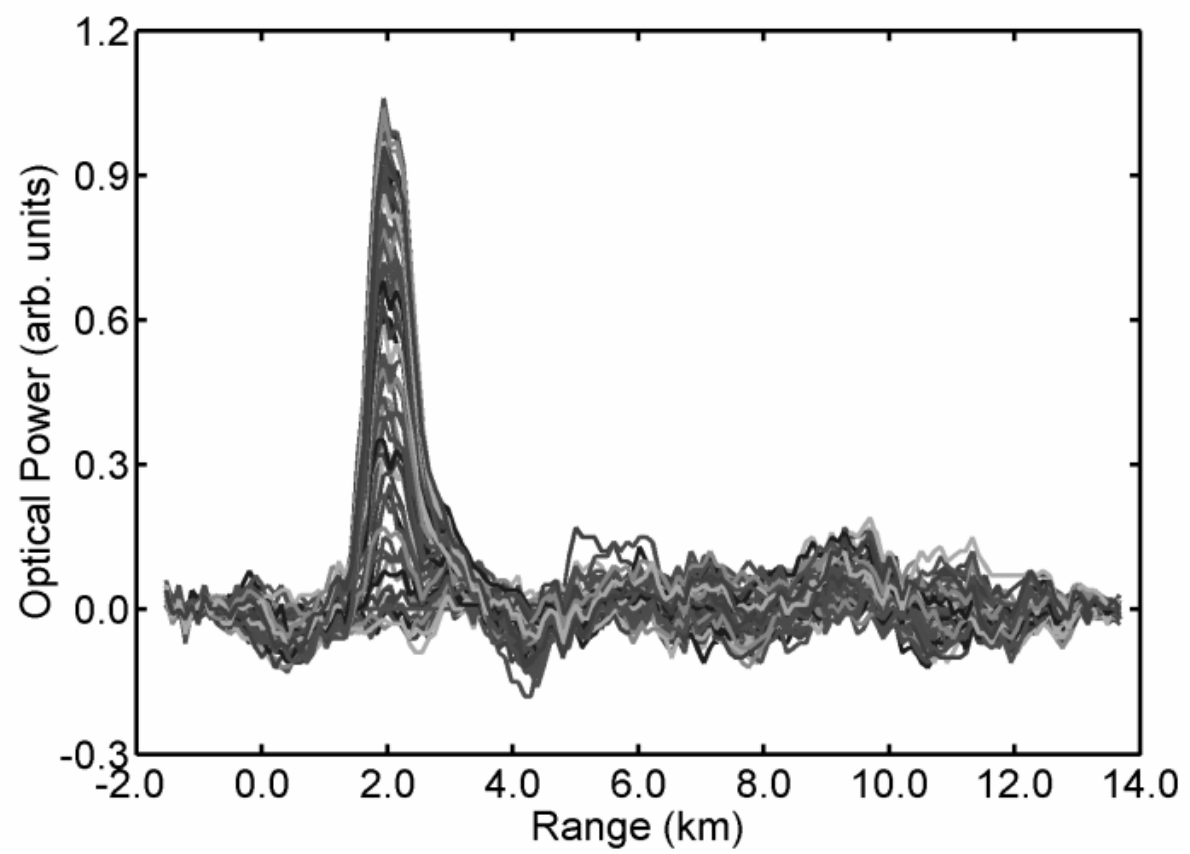

(b)

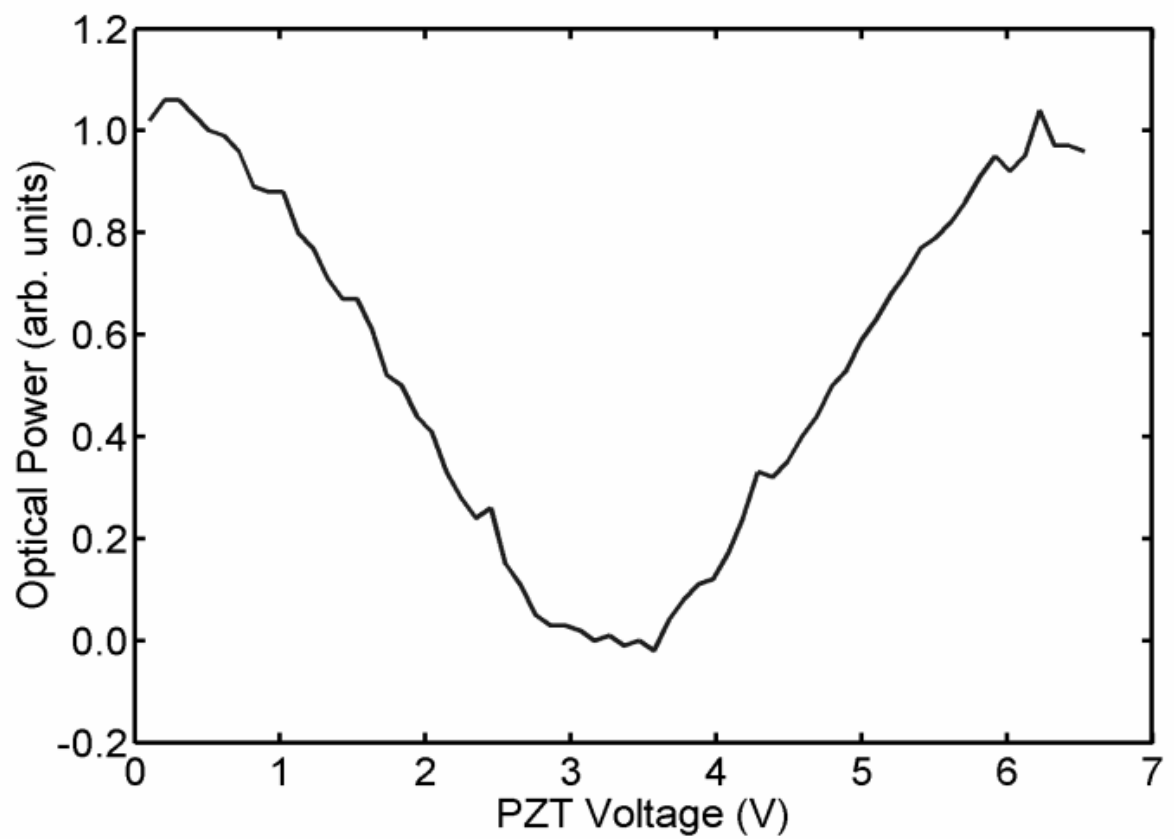

(c)

Figure 21. Continued 


\section{CHAPTER V}

\section{FIELD TESTS, SERIES 1, BRAZOS COUNTY, TX}

\section{A. Test Configuration}

Field tests to detect and locate intrusions occurring over a buried fiber optic cable were performed during the Spring of 2004 at Texas A\&M's Riverside Campus test site in Brazos County, TX. The experimental arrangement was the same as in the laboratory experiments described above, except that the PZT phase modulator was removed from the system, and in its place was spliced $44 \mathrm{~m}$ of $3 \mathrm{~mm}$-diameter single mode fiber optic cable as illustrated in Figure 22. The $44 \mathrm{~m}$ of cable passed through an insulated conduit installed in the wall of the laboratory building in which the monitoring equipment was housed. The cable was then buried in clay soil at depths of 8 to 18 inches in the configuration shown in Figure 23. A four inch wide trench was dug with a trenching machine, the cable was laid in the trench by hand, and the trench was filled with the loose dirt which had been removed from it. The cable was buried in July, 2002, and this first series of tests was carried out in early 2004. The soil had in the meantime been compacted due to the rainfall in the area (over 40 inches per year).

Data was acquired with a $60 \mathrm{MS} / \mathrm{s}$ Gage Data Acquisition Card with 2 Mega Samples of on board memory. The data was sampled at $1 \mathrm{MS} / \mathrm{s}$ and processed with a LabView system. For experiments that required continuous acquisition of $\phi$-OTDR traces at high pulse repetition rates (i.e. $>200 \mathrm{~Hz}$ ), the data had to be acquired in "Multiple Record Mode," which only saved the data to the on board memory of the 
Gage card and did not allow transfer of it to the PC until acquisition was complete. Thus, data had to be acquired in a "single shot" for a set amount of time or until the on board memory was full. By varying the pulse period to the EOM, the sensor could be interrogated for varying amounts of time, limited only by the amount of on board memory.

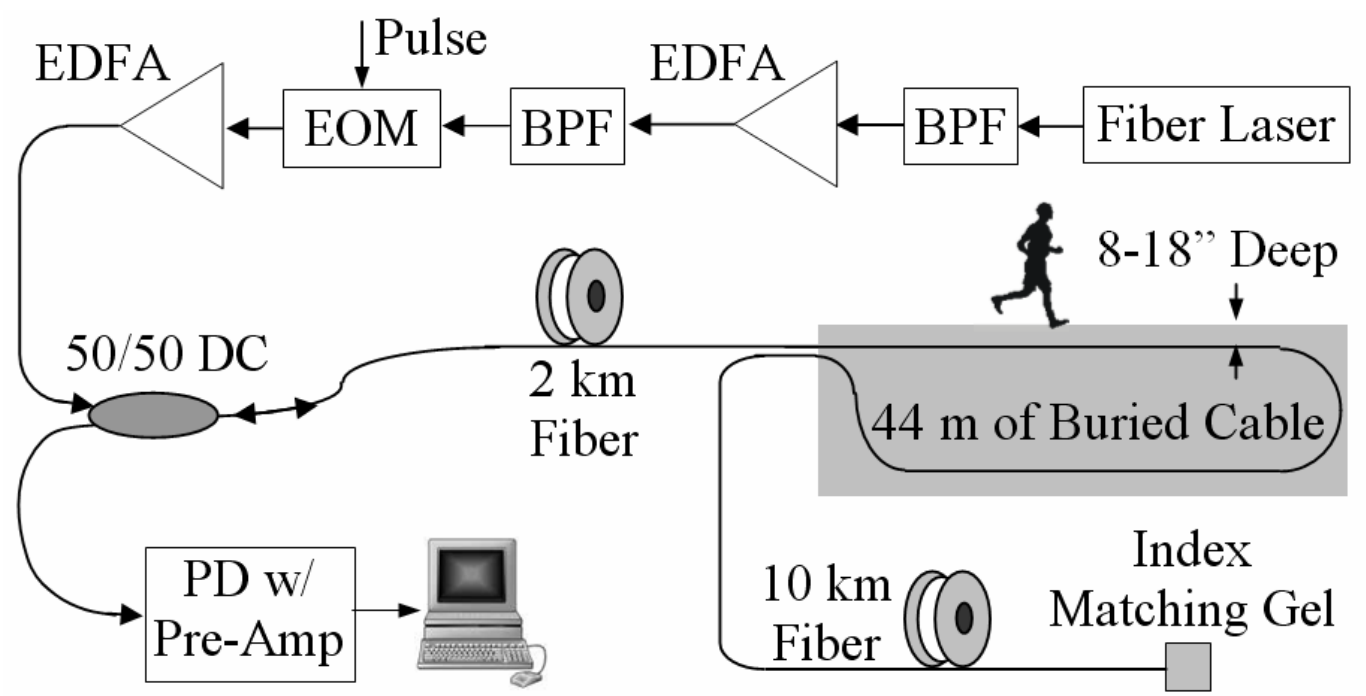

Figure 22. Preliminary field tests setup in clay soil for characterizing the $\phi$-OTDR system 


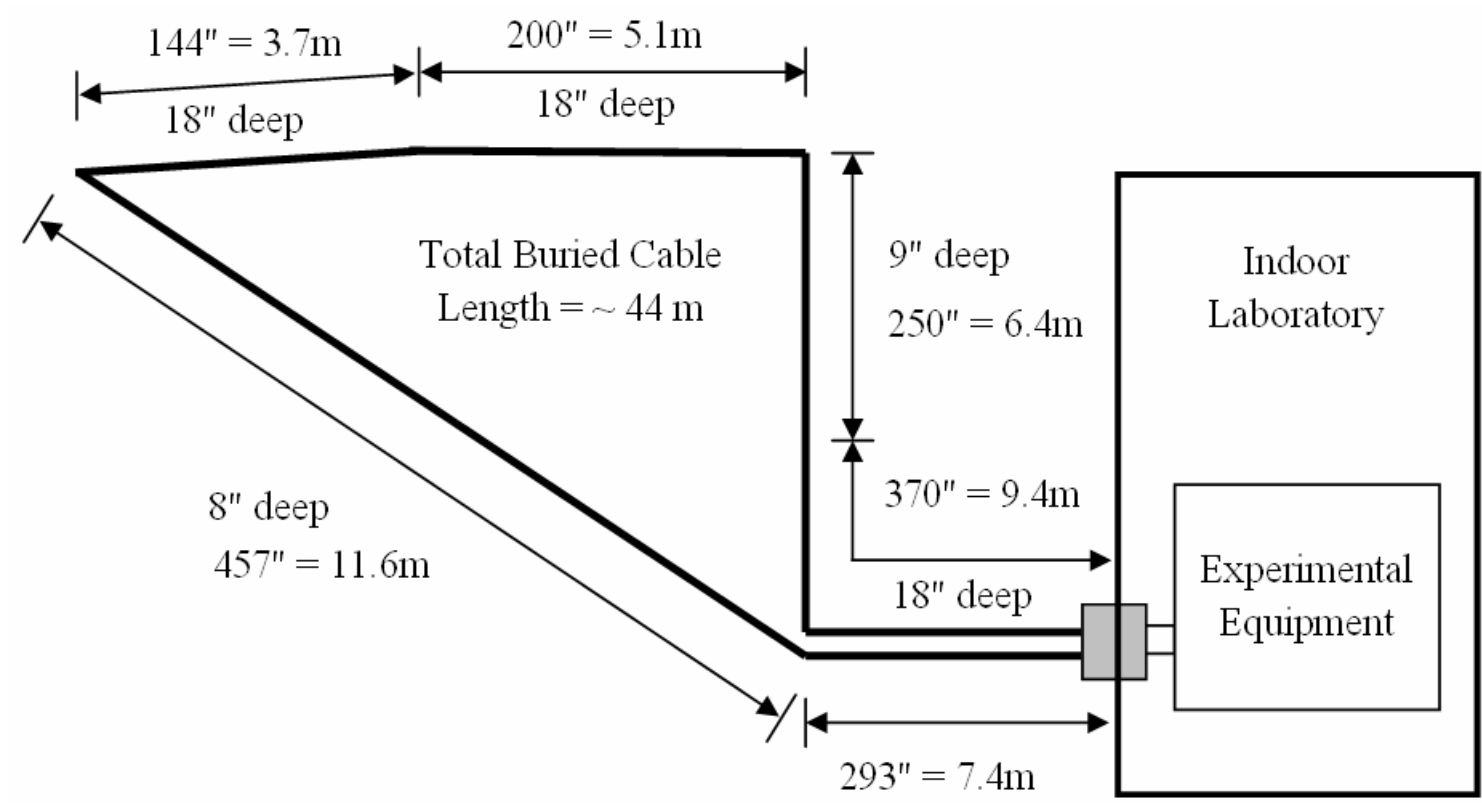

Figure 23. Configuration of buried sensor

\section{B. Test Results}

As one illustration of the phase response of the buried cable, $\phi$-OTDR traces acquired before and after an $80 \mathrm{~kg}$ person has stepped on the ground above the cable, as well as the difference of the two waveforms, are shown in Figure 24. As in the laboratory test, the width of the laser pulse entering the fiber was $10 \mu \mathrm{s}$. Also, as in the laboratory results of Figures 20 and 21, the $2 \mathrm{~km}$ range at which the response appears is the distance from the proximal end of the fiber to the location of the phase change. As expected, the difference waveform illustrates a phase change at the $2 \mathrm{~km}$ range from the start of the $\phi$-OTDR trace due to the phase-change inducing pressure created by the person's step. 


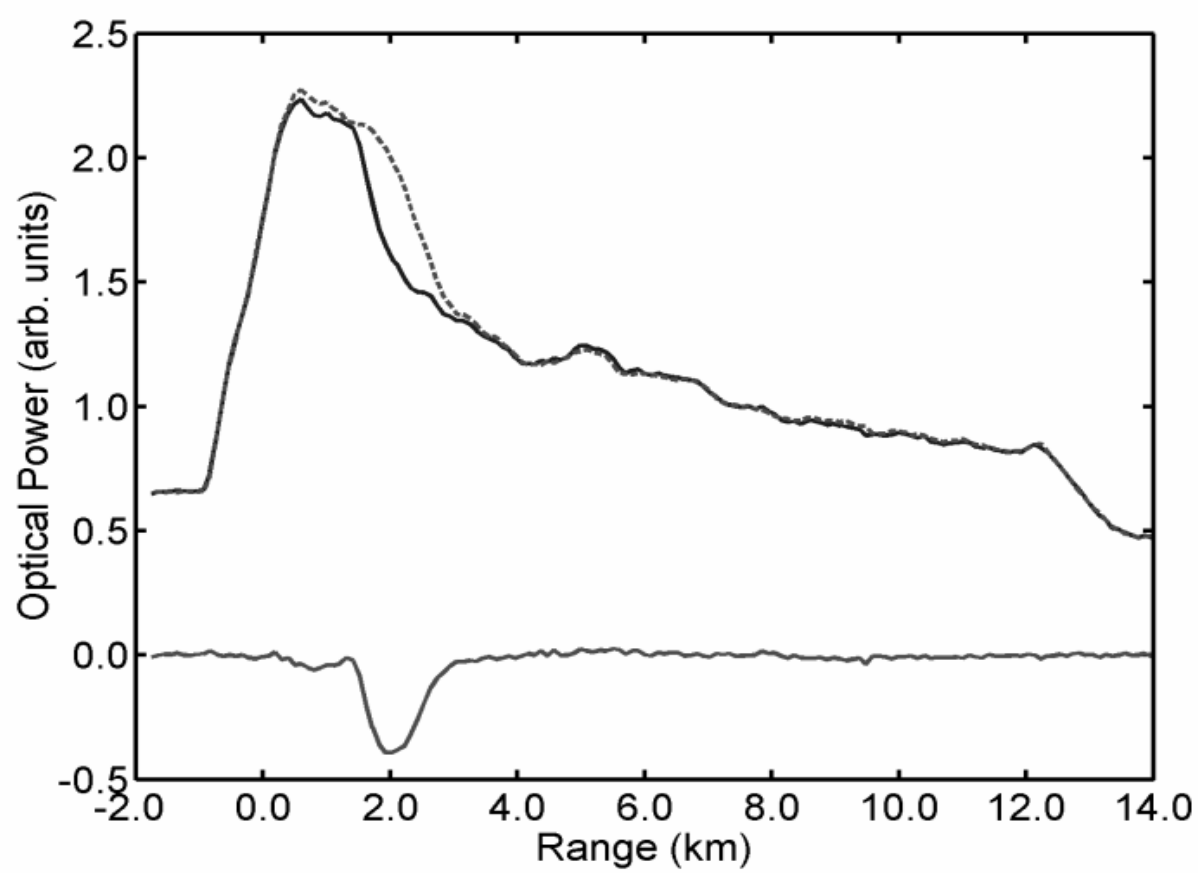

Figure 24. $\phi$-OTDR traces acquired before and after an $80 \mathrm{~km}$ person has stepped on the ground above the cable

In another experiment, the superposition of 200 consecutive $\phi$-OTDR traces with an EOM pulse width of $10 \mu$ s and a period of $300 \mu$ s were collected over a time period of $60 \mathrm{~ms}$ as a person walked back and forth across the buried cable is shown in Figure 25(a). Changes in the envelope of the traces at the $2 \mathrm{~km}$ range are visible, however, difference plots generated by subtracting the latest $\phi$-OTDR trace from a running average of the last 10 traces show the intrusion effect with greater clarity [(Figure 25(b)]. Once again, like in Figure 21(b), the spatial resolution is about $1 \mathrm{~km}$.

Finally, in an experiment to study the temporal response of different ranges to individual steps taken by a person walking on the ground above the buried cable, $\phi$ OTDR traces with an EOM pulse with of $10 \mu \mathrm{s}$ and a period of $2 \mathrm{~ms}$ were collected to 
allow for multiple seconds worth of data to be studied. In Figure 26 the temporal dependence of the $\phi$-OTDR response at three different ranges $(1,2$, and $3 \mathrm{~km})$ to individual steps is shown. In this particular case, three steps can be observed in the trace corresponding to a range of $2 \mathrm{~km}$, which commence at times of approximately 0.25 , 0.85, and 1.60 seconds from the beginning of the data record. As expected, the response is only in evidence at the $2 \mathrm{~km}$ range corresponding to the location of the intruder, while the responses for the 1 and $3 \mathrm{~km}$ ranges, which are outside the spatial resolution of the sensor, only vary slowly and randomly due to the frequency drift of the laser. For each of the observed steps, the interference pattern traverses at least four fringes, corresponding to a phase shift $\geq 8 \pi$ radians. This provides a good indication of the large sensitivity of the buried cable and is in good agreement to the previous experiments discussed in the background section on the Pressure Response of a Buried Cable. 


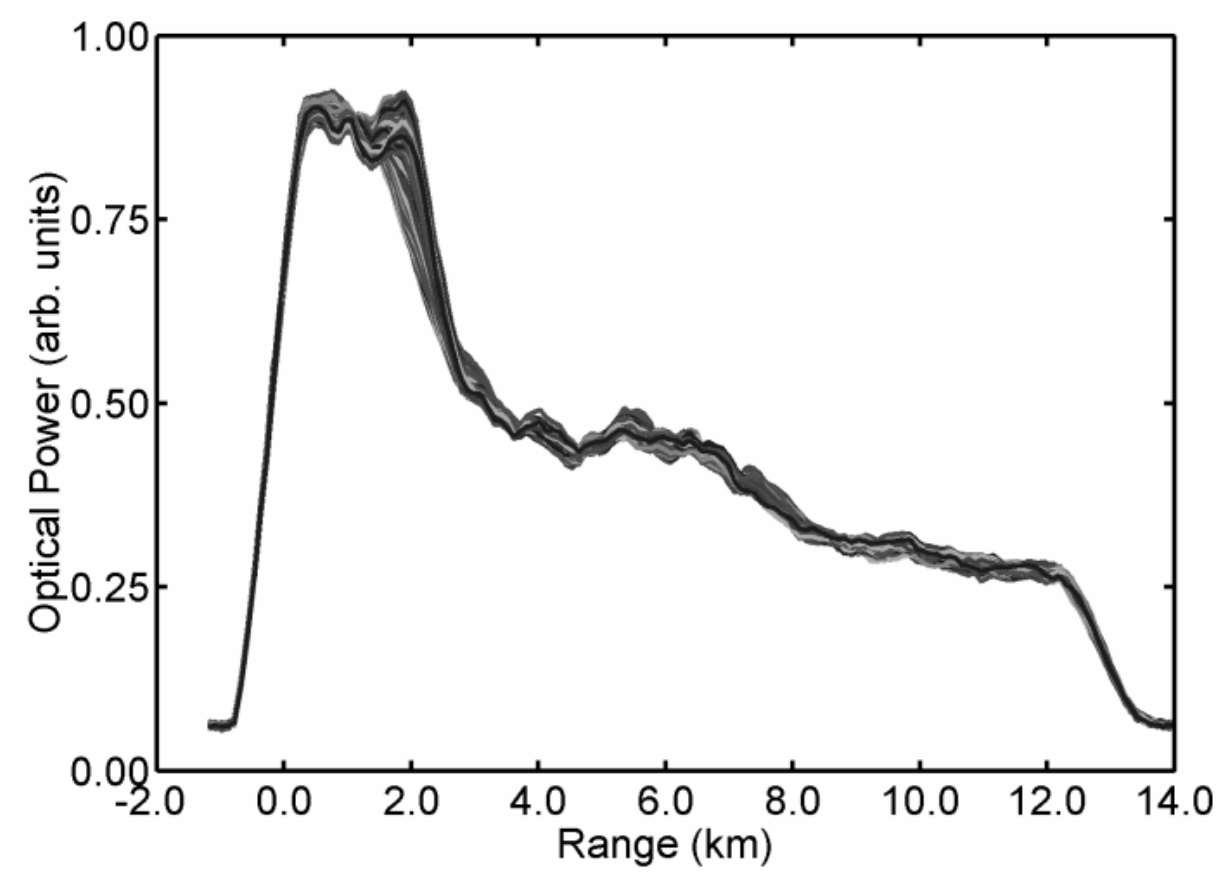

(a)

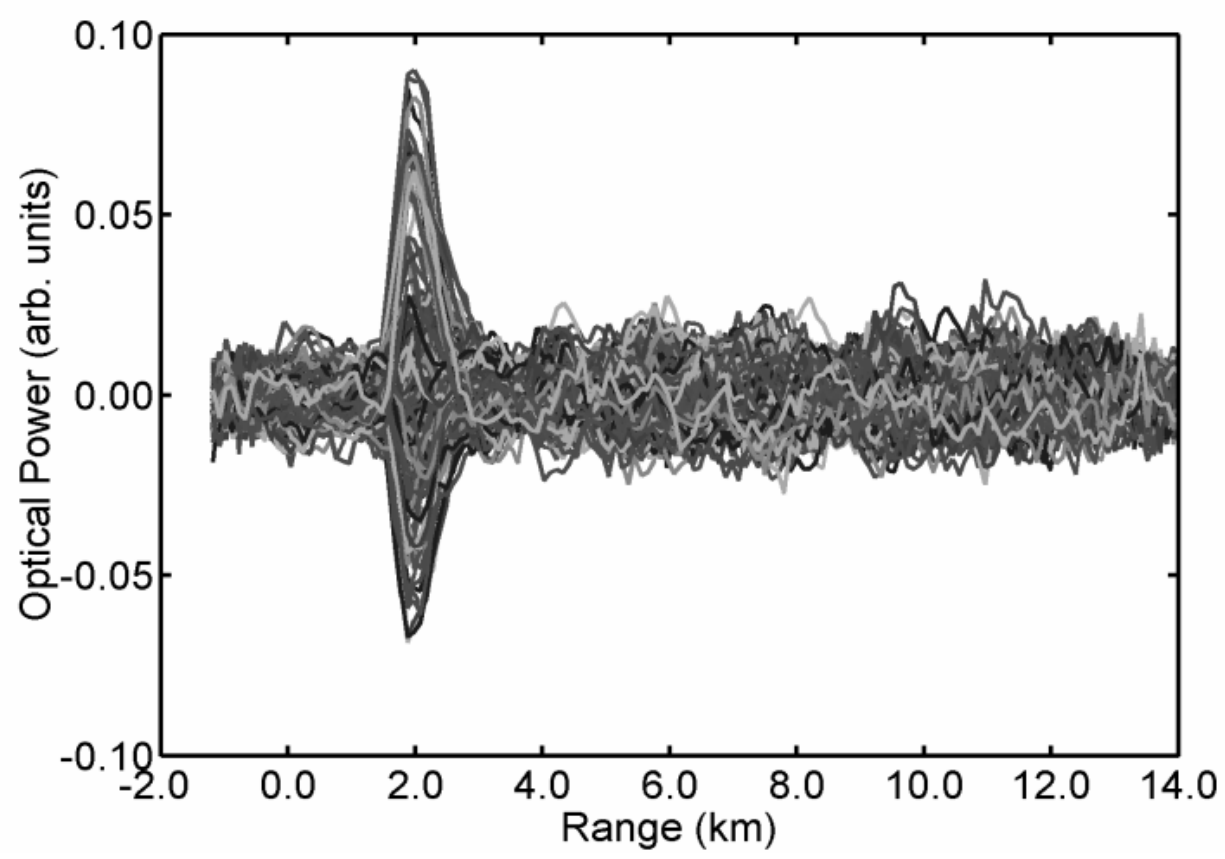

(b)

Figure 25. (a) Superposition of $\phi$-OTDR traces produced in response to a person walking on the ground above the buried cable; (b) difference plots of the same data. 


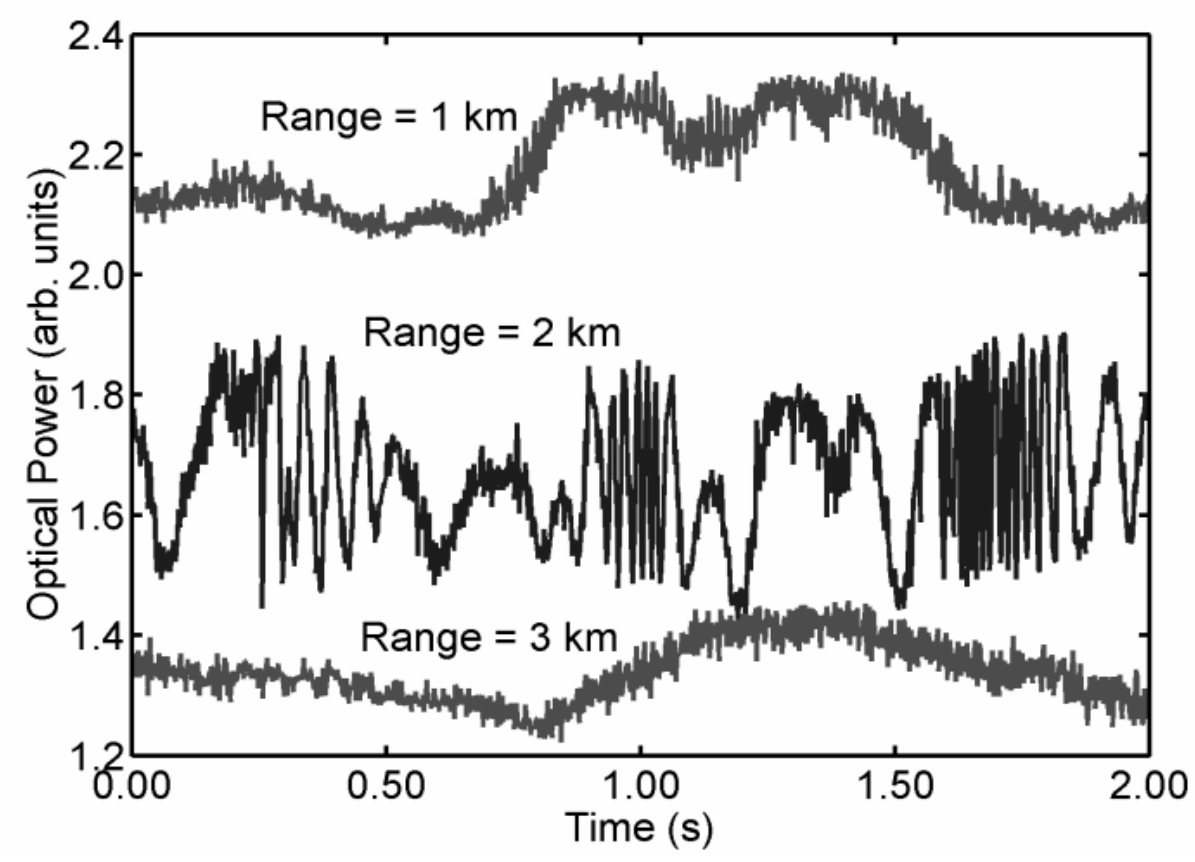

Figure 26. Response of $\phi$-OTDR over a time period of 2 seconds at ranges of 2, 3, and $4 \mathrm{~km}$ when a person is walking on the ground above the buried fiber cable at the $2 \mathrm{~km}$ location. 


\section{CHAPTER VI}

\section{FIELD TESTS, SERIES 2, YUMA, AZ}

\section{Test Configuration}

A field demonstration of the distributed fiber optic sensor over a long buried cable run $(>10 \mathrm{~km})$ in desert terrain at the U.S. Marine Corps Air Station in Yuma, AZ was performed during the Summer of 2004 using the experimental $\phi$-OTDR arrangement in Figure 27. This is a modified version of the arrangement illustrated in Figure 22, which now uses one of the optical amplifiers (EDFAs) to boost the signal at the receiver end and incorporates a Polarization Beam Splitter (PBS) and a second receiver, to split the light into orthogonal polarizations and process them separately. These changes provide enhanced signal-to-noise ratio (SNR) in the receivers, greater visibility in the $\phi$-OTDR interference pattern, and reduce the probability of signal "fading." The SNR was affected because the second EDFA (after the EOM) in the previous configuration became saturated by the first, resulting in decreased visibility of the interference pattern in the $\phi$-OTDR due to increased amplifier stimulated emission (ASE), which is non-coherent. Removing the first EDFA solved this problem but led to decreased optical power in the sensing fiber; Thus this EDFA was moved to the receiver end of the setup to increase the system SNR

The $12 \mathrm{~km}$ simulated sensor was replaced with $8.5 \mathrm{~km}$ of two-fiber, $4.5-\mathrm{mm}$ outdoor (OCC) cable buried in desert soil in a $1 \mathrm{ft}$ deep, $2.5 \mathrm{ft}$ wide triangular trench 
filled with loose sand. The monitored length of fiber was extended to $19 \mathrm{~km}$ by splicing the two fibers at the end of the buried cable in a "loop back" arrangement and adding to the input side a $2 \mathrm{~km}$ reel of fiber that was thermally and acoustically insulated in the control building where the monitoring equipment was located. Except for the first 0.3 $\mathrm{km}$ of cable nearest the control building, the $8.5 \mathrm{~km}$ of buried cable utilized in the tests was buried parallel to a one-lane paved road, at distances ranging from $2.4 \mathrm{~m}$ to $6.1 \mathrm{~m}$ from the edge of the pavement.

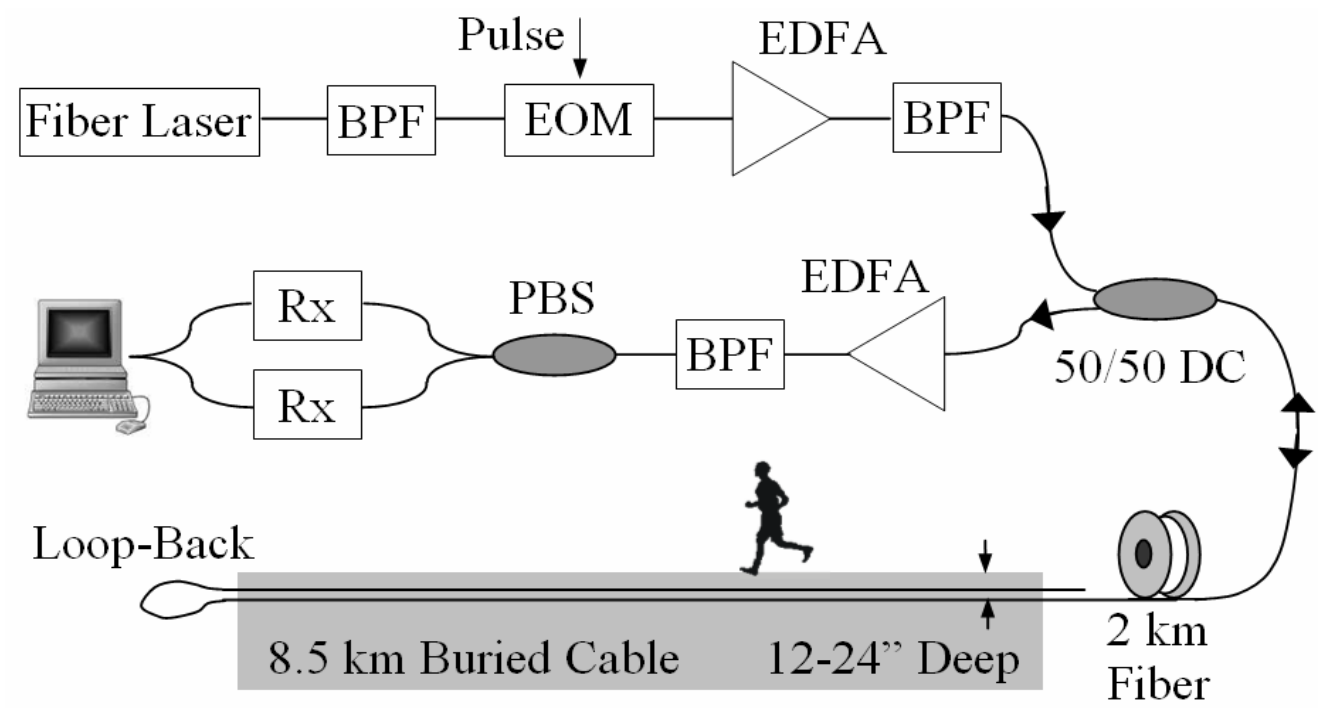

Figure 27. Field tests setup in desert terrain for characterizing the $\phi$-OTDR system

The light source was also modified to the configuration depicted in Figure 28 for increased frequency stability, optical power, and coherence (i.e. the ratio of coherent vs. non-coherent power at its output). $\mathrm{The}^{+{ }^{+3}}$ doped fiber gain medium was replaced with higher gain fiber of $18 \mathrm{~dB} / \mathrm{m}$ to achieve higher output power levels. The $980 \mathrm{~nm}$ pump laser was directly spliced into the backside FBG to forward pump the fiber laser to reduce ASE, which prevents pump power from being used to amplify the desired 
coherent light. The optical output power from this laser is up to $500 \mu \mathrm{W}$, and the emission wavelength is again $1555.4 \mathrm{~nm}$, corresponding to the reflectance peak of the FBGs.

The short feedback loop was later removed because in this new configuration, the laser exhibited multimode behavior or frequent mode hopping $(<20 \mathrm{sec})$ which greatly reduced the sensitivity to intruders vs. the single mode case, even when a person walked directly over the buried cable. Additionally, the 90/10 DCs were now unnecessary which upon their removal reduced the intracavity optical loss. Finally, a commercial ice chest (100 qt.) and foam board insulation was now utilized to house the laser and better insulate against environmental disturbances.

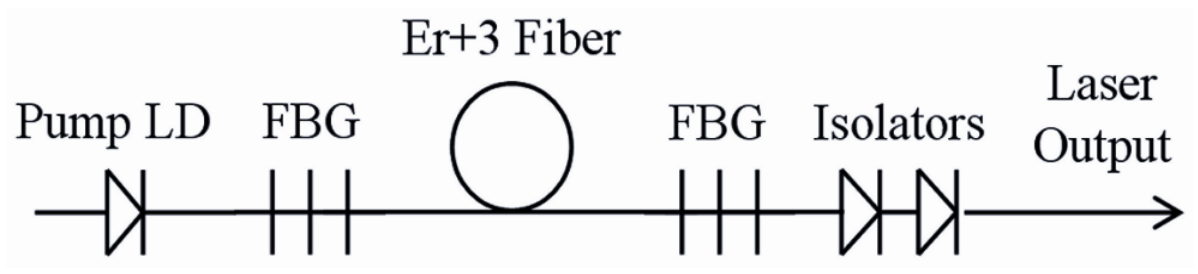

Figure 28. Experimental setup for fiber laser used as the light source in Yuma, AZ field tests

The EOM was now driven with $2 \mu$ s pulses for enhanced spatial resolution (200 $\mathrm{m}$ ) in the sensor, while the receivers now operated at a $20 \mathrm{~dB}$ gain and a $700 \mathrm{kHz}$ bandwidth to better detect the increased interference visibility in the $\phi$-OTDR traces. Data was continuously acquired with a new $50 \mathrm{MS} / \mathrm{s}$ Gage Data Acquisition Card sampling at $5 \mathrm{MS} / \mathrm{s}$. The OTDR traces were then processed with a PC in real-time at pulse repetition rates of $\sim 5 \mathrm{~ms} /$ pulse using a $\mathrm{C}^{++}$program and displayed graphically along with the intrusions in LabView as shown in Figure 29. This is a screen capture 
from a video recording of the LabView plots simultaneously displaying the two independent $\phi$-OTDR traces (one for each orthogonal polarization) on top in red and white and the processed signal on bottom. To aid in reducing the "missed intruder" probability, the processed waveform is composed of a high-bandwidth signal component (upward going trace) and a low-bandwidth component (downward going trace), for both polarizations. The color of the processed signal components correspond to the respective polarization displayed on top. Phase changes were detected by subtracting the latest OTDR trace from a previously stored trace. The identifying label on the upper right hand corner indicates the video tape number ("B12"), the starting time of the video clip relative to the start of the tape in minutes and seconds (" $13 \mathrm{~m} 06$ "), and the time of the frame relative to the start of the clip in seconds and 30ths of a second ("39-16").

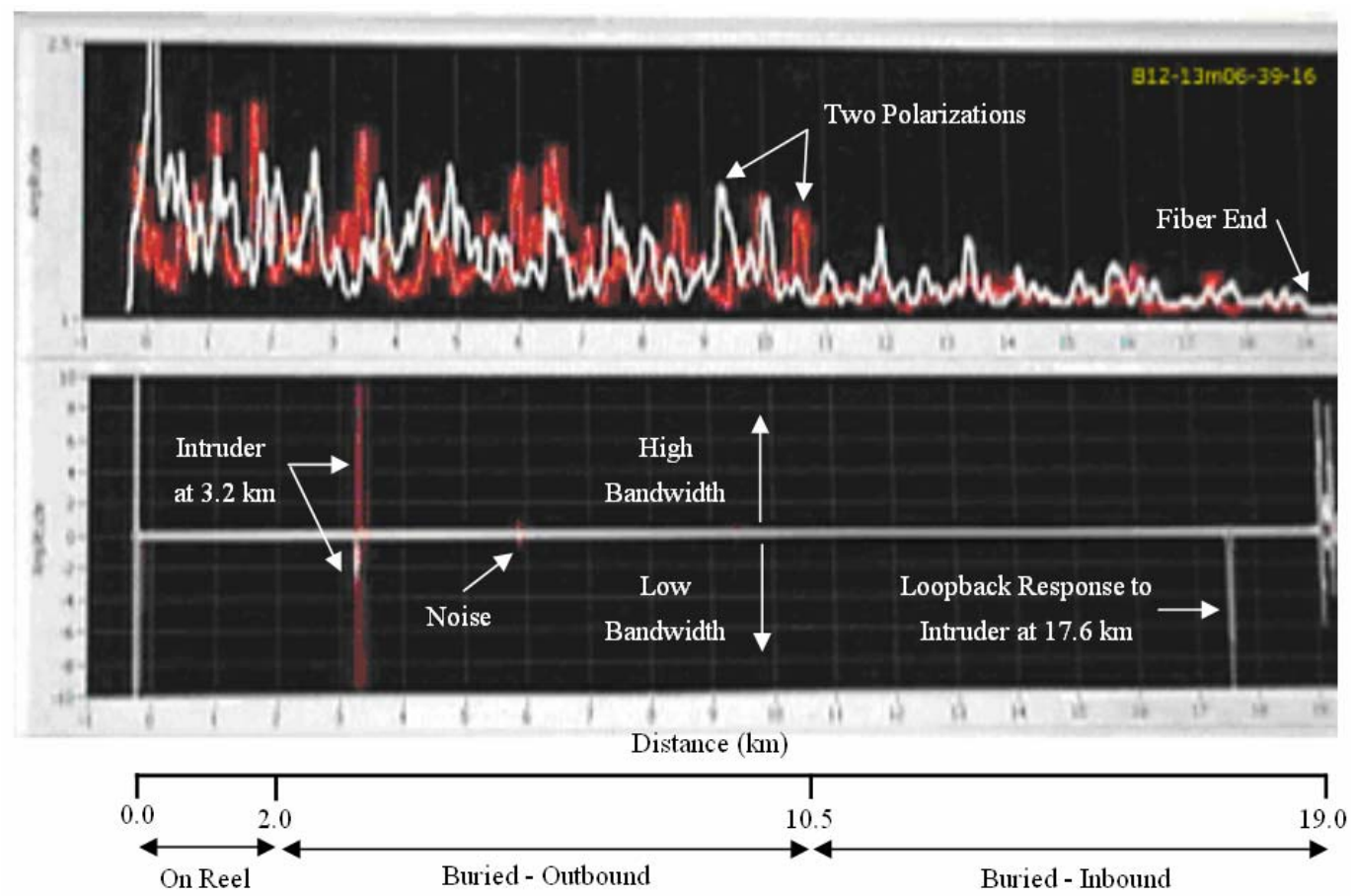

Figure 29. Sample screen capture of PC with both OTDR traces and processed signal 


\section{Results - Human Intruders}

Once the burial of the $8.5 \mathrm{~km}$ cable was accomplished and extended to $19 \mathrm{~km}$ as explained in the previous section, the performance of the $\phi$-OTDR was investigated with humans and vehicles. As one illustration of the phase response of the buried cable, an 80 $\mathrm{kg}$ person walked along the length and across the buried sensor at a range of $5.4 \mathrm{~km}$ and a loopback range of $15.6 \mathrm{~km}$. A typical result recorded from the PC screen before, during, and after a particular step taken by the intruder is shown in Figure 30. For each such event, the observed processed signal is rated on a ten point scale. For the activity at a range of $5.4 \mathrm{~km}$, the signal level from Figure 30(b) rates a 9 for the red polarization for both up-going (high bandwidth) and down-going (low bandwidth) signals, a 3 for the upgoing signal for the white polarization, and a 4 for the down-going signal for that polarization. The difference in the signal scores proves the advantage of adding redundancy to the system by observing and processing each polarization as independent signal channels. This reduces the probability of a "missed intruder" in cases that phase changes are not very visible in a single polarization due to fading.

Other peaks visible in the processed signals are noise arising from a synchronization problem at that time that produced "ghost" phase-shifts in the signal processing. As indicated by the labels on the video frames, the elapsed time between Figure 30(a) and (b) is $0.30 \mathrm{sec}$ and between Figure 30(b) and (c) is $0.27 \mathrm{~s}$. The effect of the intruder on the loopback portion of the fiber at a range of $15.6 \mathrm{~km}$ is not detected in this case. 


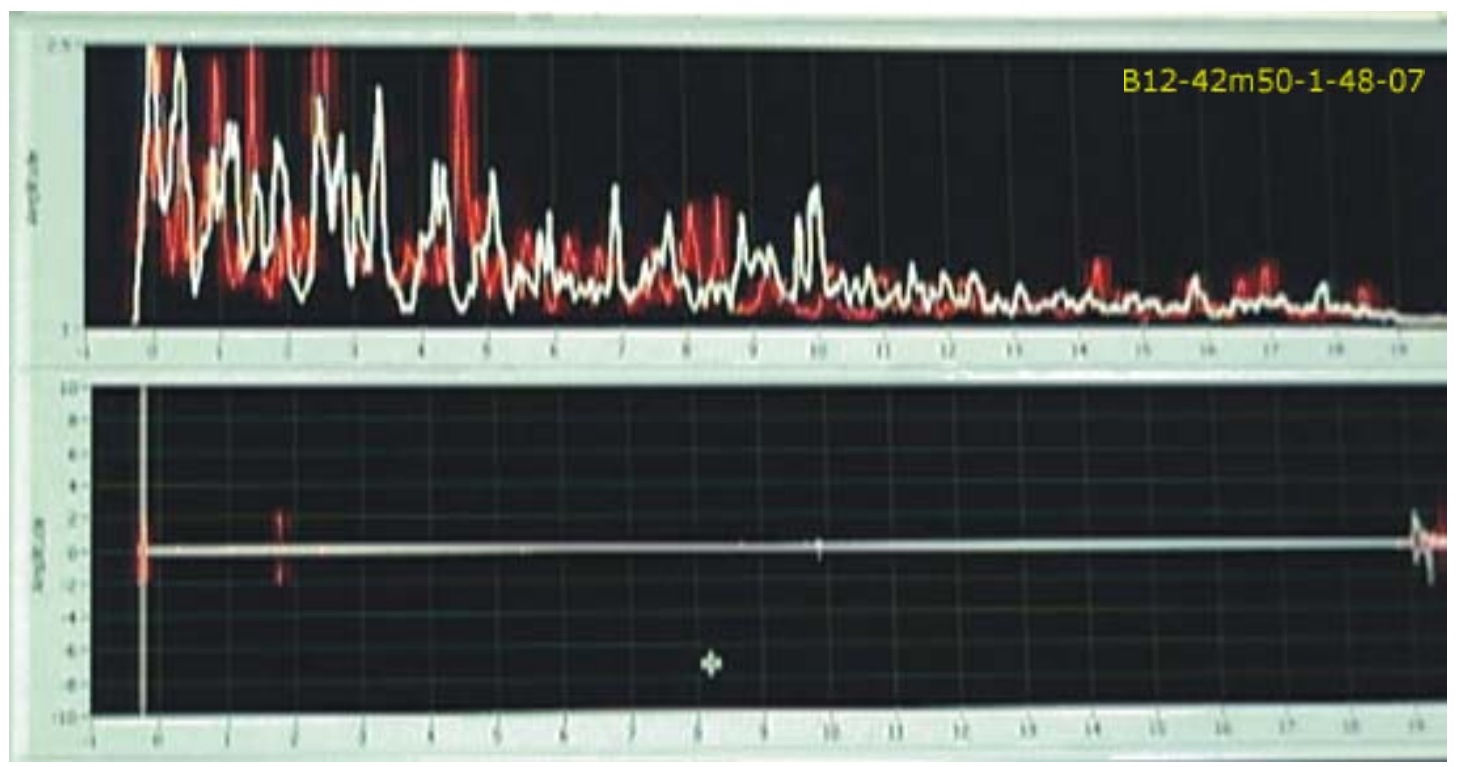

(a)

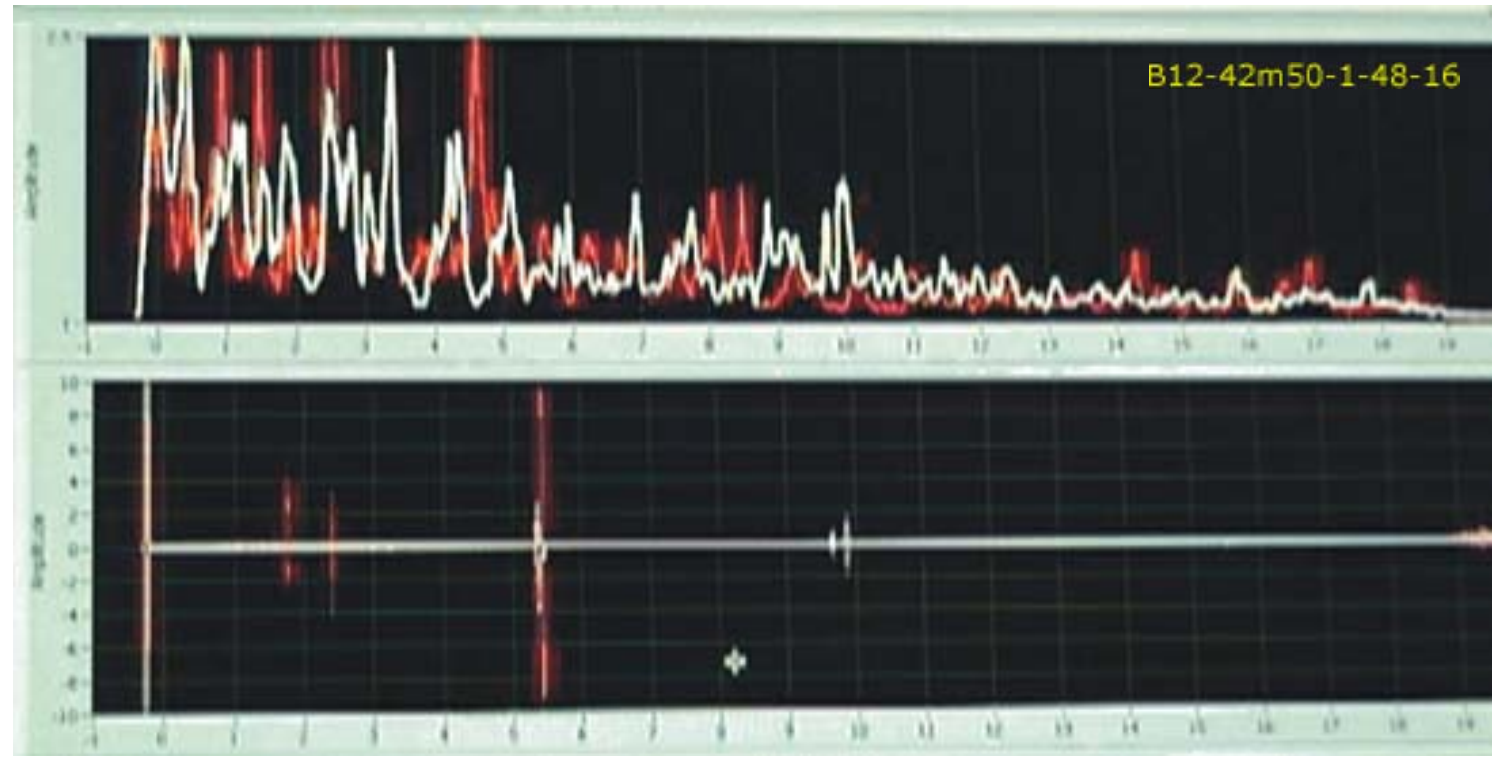

(b)

Figure 30. Results of an intruder taking a step at $5.4 \mathrm{~km}$ range. As indicated by the labels on the video frames, the elapsed time between (a) and (b) is $9 / 30 \mathrm{~s}(0.30 \mathrm{~s})$ and between (b) and (c) is $8 / 30 \mathrm{~s}(0.27 \mathrm{~s})$. The effect of the intruder on the loop-back portion of the fiber at a range of 13.6 $\mathrm{km}$ is not detected in this case. 


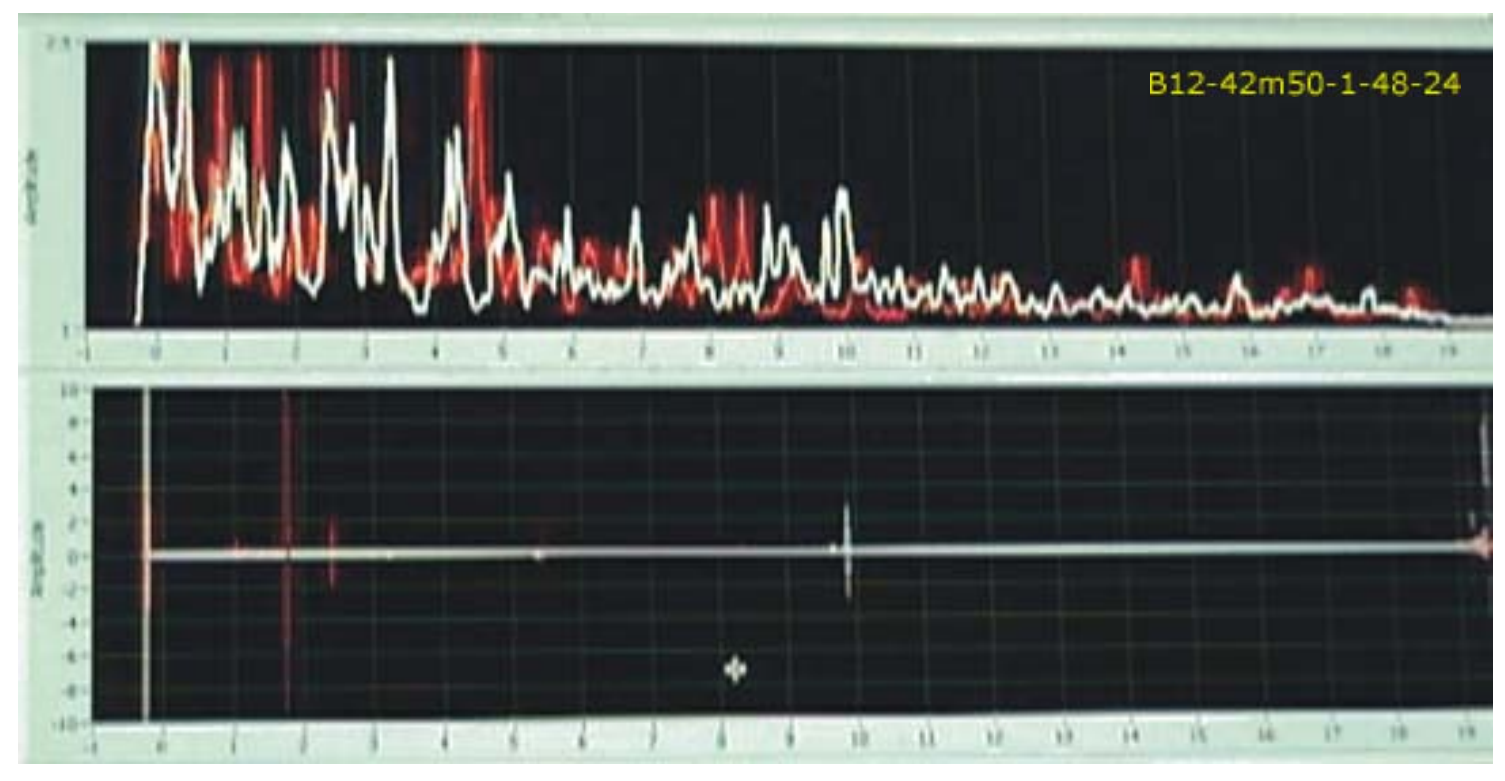

(c)

Figure 30. Continued

Examples in which a strong signal is observed in both outbound and inbound fibers are given in Figures 31-33. In Figure 31, the scores produced by an intruder's step at the $3.3 \mathrm{~km}$ range for up-going and down-going traces were 9 and 10 for the red polarization, and 0 and 3 for the white polarization. At the $17.7 \mathrm{~km}$ range, the score was 10 for the down-going trace for the white polarization, and 0 for the other 3 cases.

Figure 32 and 33 are examples at range of $5.4 \mathrm{~km}$ and a loopback range of 15.6 $\mathrm{km}$. In Figure 32, the larger signal is observed in the outbound case, while the opposite is true in Figure 33. For all these cases, at both ranges, one polarization strongly picked up the intrusion while the other hardly did, and vice versa. This provides further proof that the redundancy of using both polarizations reduces the probability of a "missed intruder" 


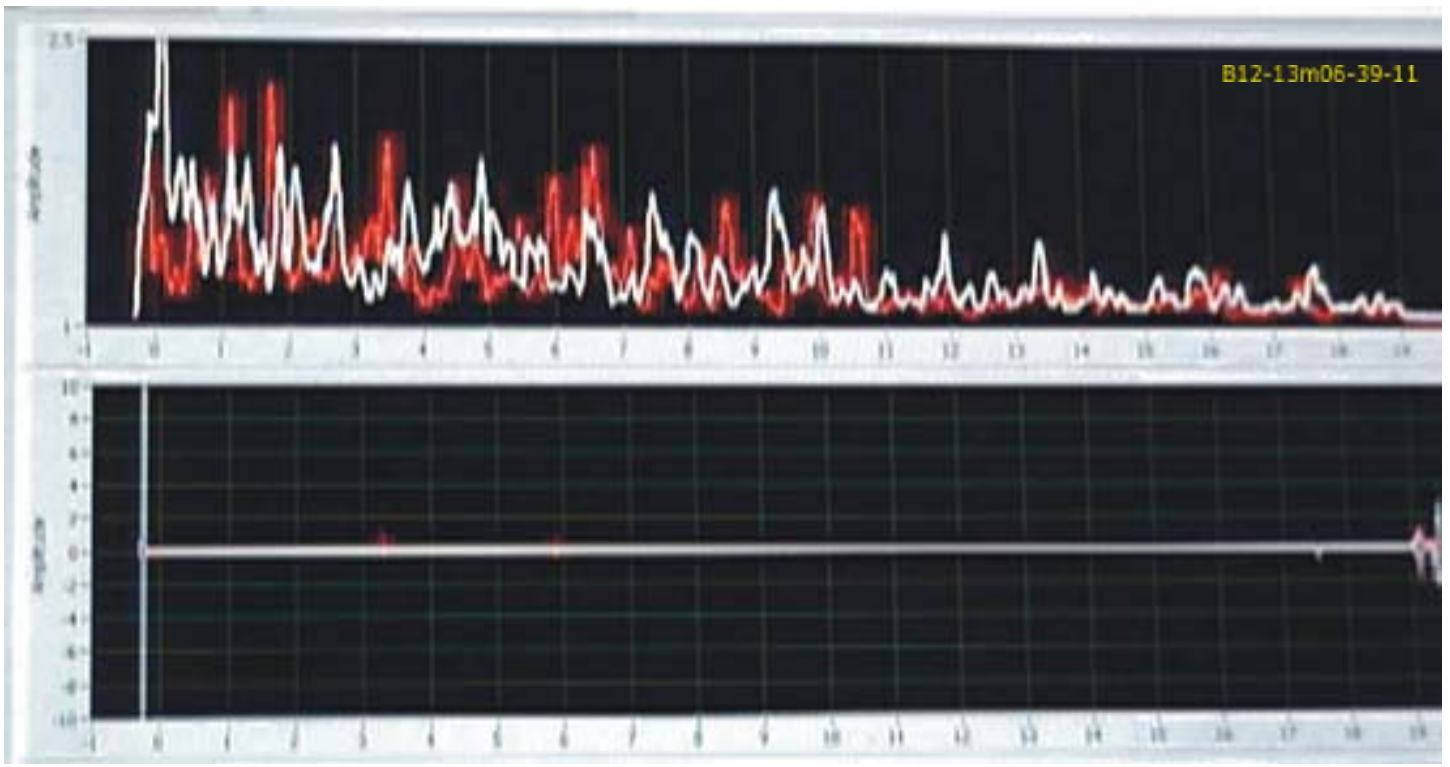

(a)

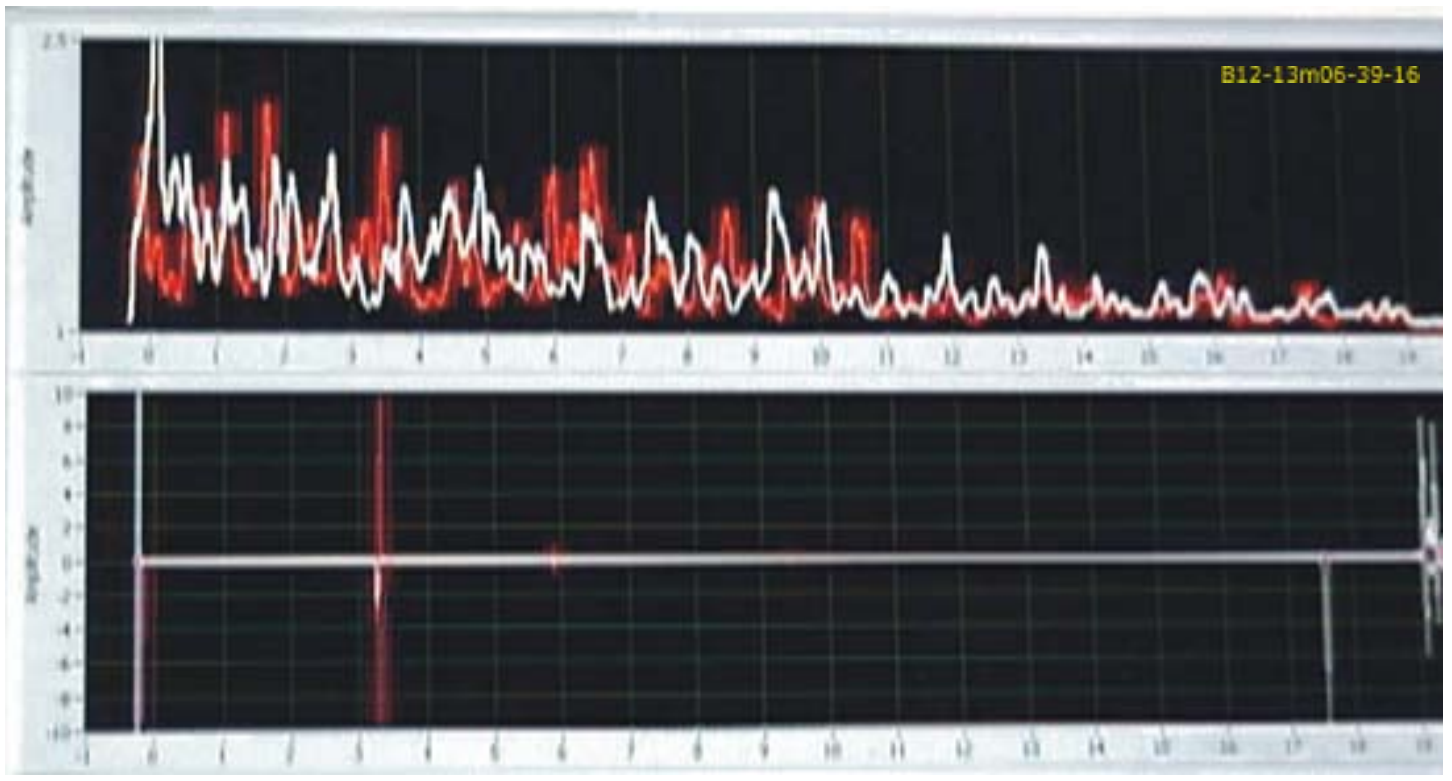

(b)

Figure 31 . Response to an intruder taking a step at a range of $3.3 \mathrm{~km}$. The loopback signal is seen in the white processed signal waveform at a range of $17.7 \mathrm{~km}$. 


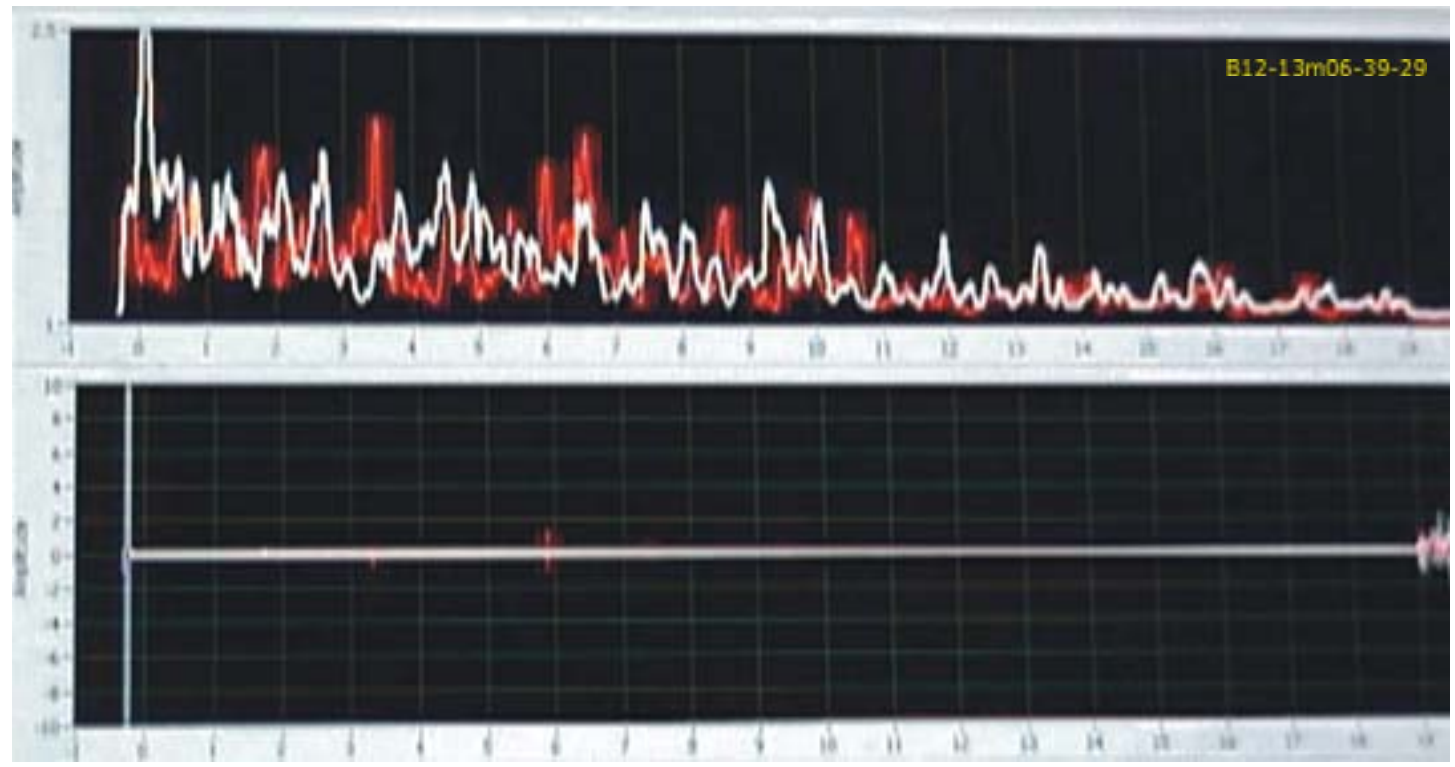

(c)

Figure 31. Continued

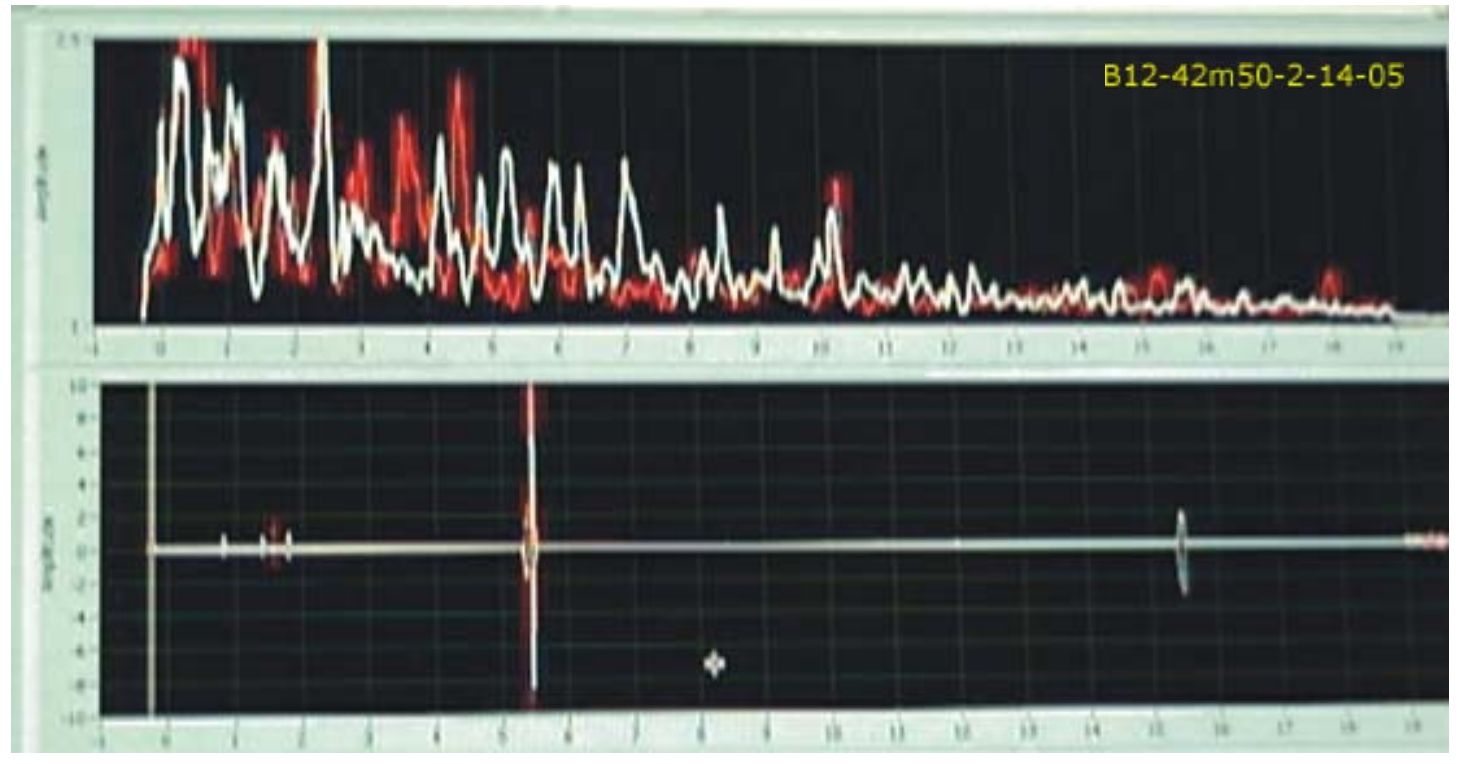

Figure 32. Response to an intruder taking a step at a range of $5.4 \mathrm{~km}$ with a strong signal in the outbound fiber 


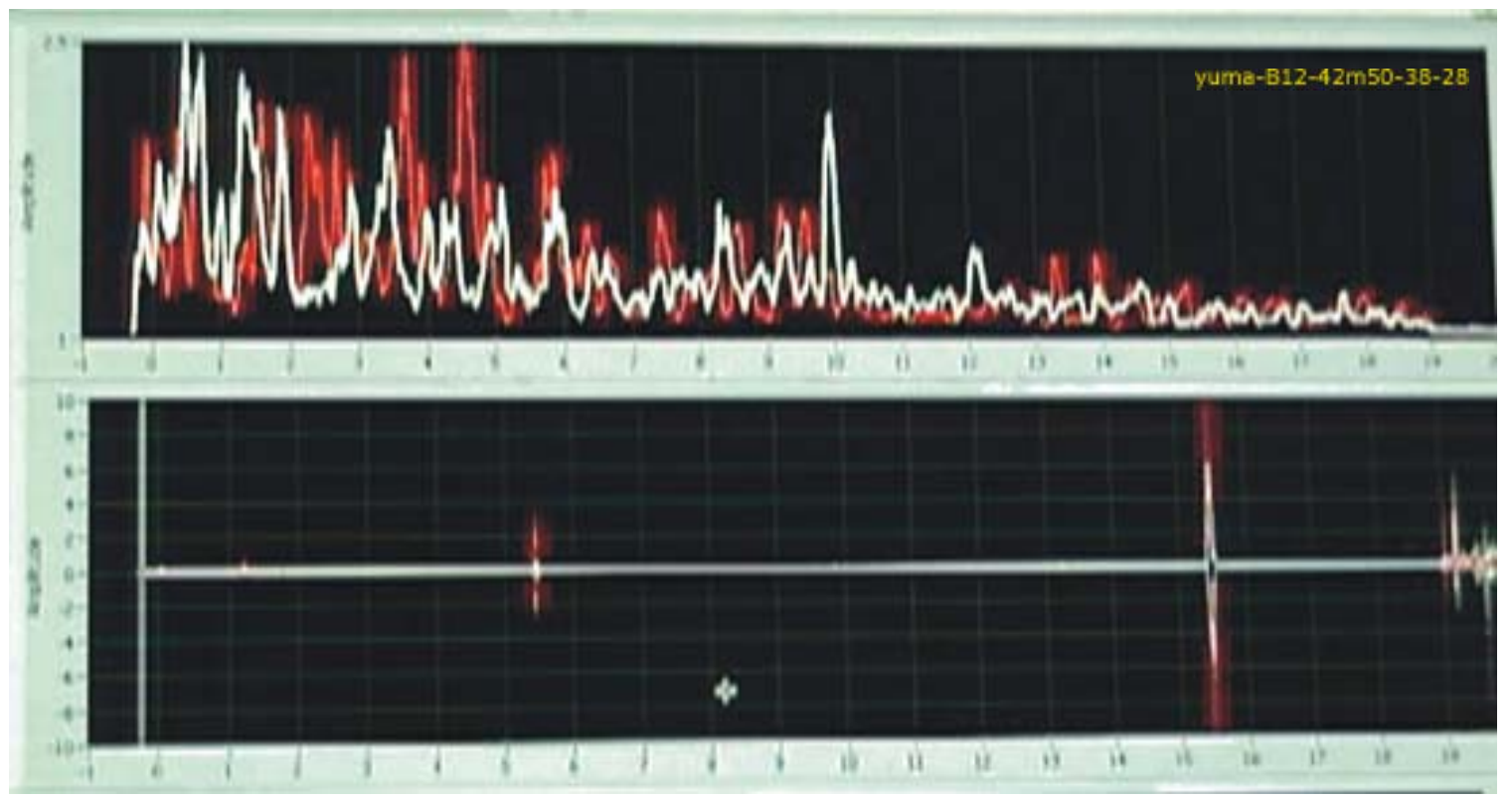

Figure 33. Response to an intruder taking a step at a range of $5.4 \mathrm{~km}$ with a strong signal in the inbound fiber

\section{E. Results - Vehicles}

In another test, the system response was observed on the PC screen as an automobile weighing about 3000 pounds traveled down the road from a location near the end of the $8.5 \mathrm{~km}$ of buried cable back to the control building, at an average speed of 45 mph. In this case, the phase changes in the sensing fiber result from seismic waves generated when the automobile drives over small rooks and imperfections in the road.

A system to characterize the performance of the sensor was developed based on the number of detections determined for each kilometer of road. "High threshold" and "moderate threshold" detections were tracked. A "high threshold" detection was taken to be an event having an amplitude of full scale (i.e. a score of 10) for either the up-going or down-going processed trace for either of the two polarizations; a "moderate 
threshold" detection required an amplitude of $2 / 5$ of full scale (i.e. a score of 4 ) or more for one of these four cases. The detection sensitivity of the system was found to be greatest at short ranges as expected because of the decreased amount of backscattered optical power from the far end of the sensor. As seen in Figure 34, the number of moderate threshold detections in each $\mathrm{km}$ decreased from a maximum of 16 at the shortest $(3 \mathrm{~km})$ range to a minimum of 3 at ranges of $15 \mathrm{~km}, 16 \mathrm{~km}$, and $18 \mathrm{~km}$. The number of high threshold detections in a $\mathrm{km}$ decreased from a maximum of 7 at a range of $4 \mathrm{~km}$ range to a minimum of 0 at a range of $15 \mathrm{~km}$.

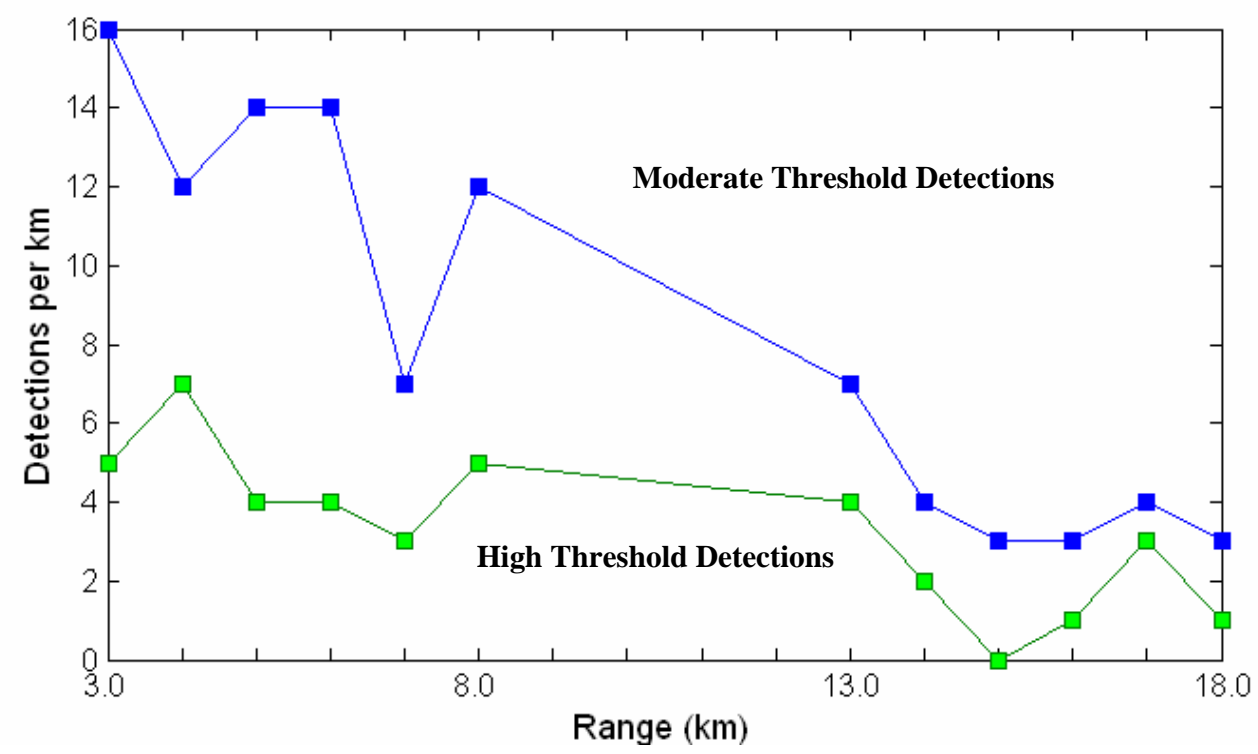

Figure 34. Response to automobile traveling on road 8' $-20^{\prime}$ from the buried sensor 


\section{CHAPTER VII}

\section{FIELD TESTS, SERIES 3, BRAZOS COUNTY, TX}

\section{A. Test Configuration}

Final field tests to further characterize the intrusion sensor system were carried out back at Texas A\&M's Riverside Campus in Brazos Country, TX, during the Fall of 2004 and Spring of 2005 using the experimental arrangement in Figure 35. This is a modified version of the arrangement used in the Yuma, AZ experiments and illustrated in Figure 27. In this case, the $19 \mathrm{~km}$ sensor was replaced once again by the simulated 12 $\mathrm{km}$ sensor consisting of the 2 and $10 \mathrm{~km}$ spools of fiber with $44 \mathrm{~m}$ of $3 \mathrm{~mm}$-diameter single mode, fiber optic indoor cable buried at a depth of 8-18 inches in clay soil. Once again the width of the laser pulse entering the fiber was $2 \mu \mathrm{s}$, giving a $200 \mathrm{~m}$ resolution.

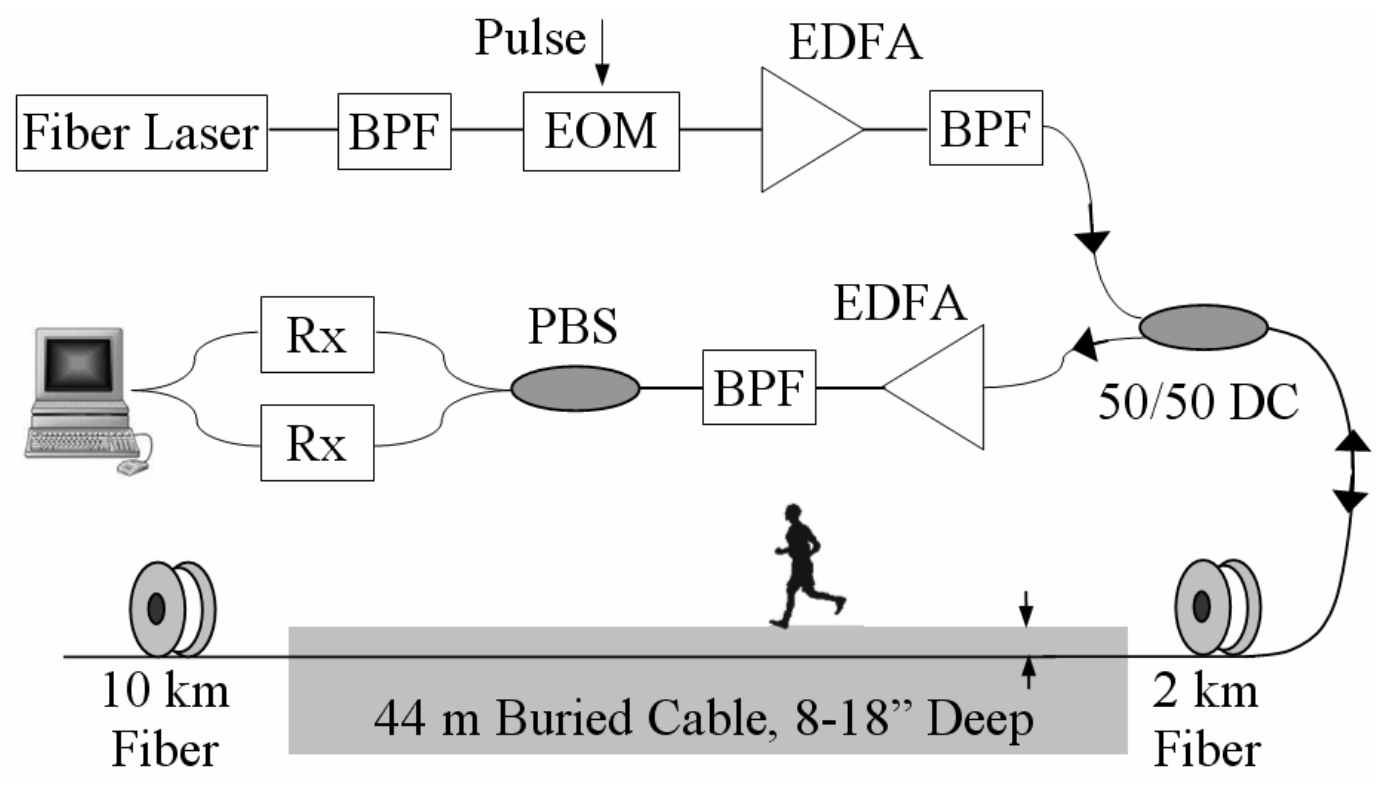

Figure 35. Field tests setup in clay soil for characterizing the $\phi$-OTDR system 


\section{B. Test Results}

As one illustration of the phase response of the buried cable, $\phi$-OTDR traces for each orthogonal polarization simultaneously acquired before and after an $80 \mathrm{~kg}$ person has stepped on the ground above the cable buried at a depth of 18 inches, as well as the difference of the two waveforms, are shown in Figures 36(a) and (b). As in the previous results in clay soil (Figures 24-25), the $2 \mathrm{~km}$ range, at which the response appears, is the distance from the proximal end of the fiber to the location of the phase change. Similar to the results seen from Yuma, AZ (Figures 30-33), the improved spatial resolution along with the reduction of ASE in the system produces more structure and higher visibility in the OTDR traces in Figures 36(a) and (b) than in the earlier results of Figures 24-25.

Lateral sensitivity was tested with an $80 \mathrm{~kg}$ individual walking towards the 18 inch deep sensor line as shown in Figure 37. The temporal dependence of the $\phi$-OTDR response to the phase changes produced by the intruder in a typical run is shown in Figure 38. Nine steps with a $2-\mathrm{ft}$ gait can be seen in both polarizations as noted in the figure as a person approaches the sensor from $15 \mathrm{ft}$ away and stopping $1 \mathrm{ft}$ after it. As expected, the response is only in evidence at the $2 \mathrm{~km}$ range corresponding to the location of the intruder. Each step in general produces a larger phase change in the interference pattern than the previous step, as the person approaches the buried sensor, with the greatest change in the last two steps, corresponding to $1 \mathrm{ft}$ before and after the location of the buried sensor. 


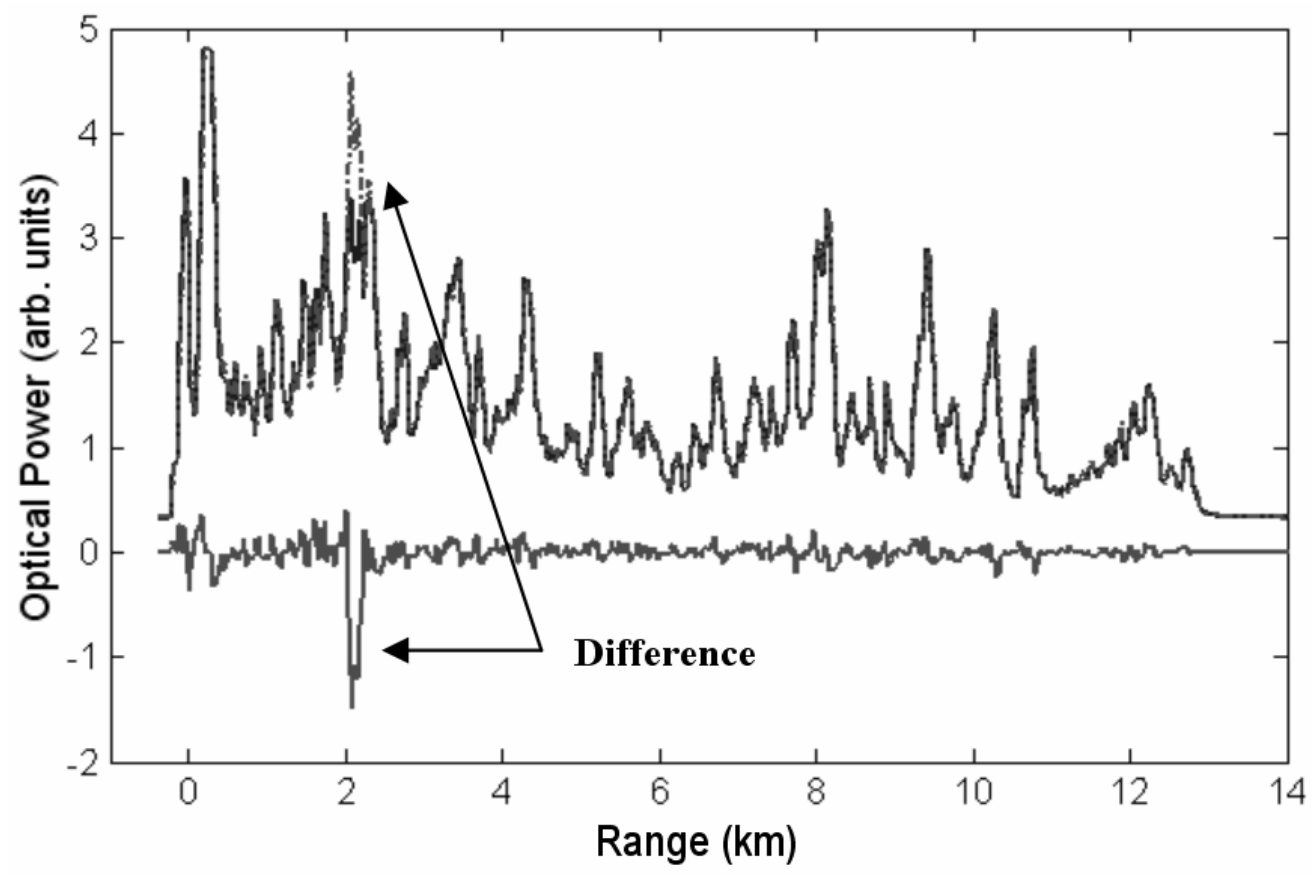

(a)

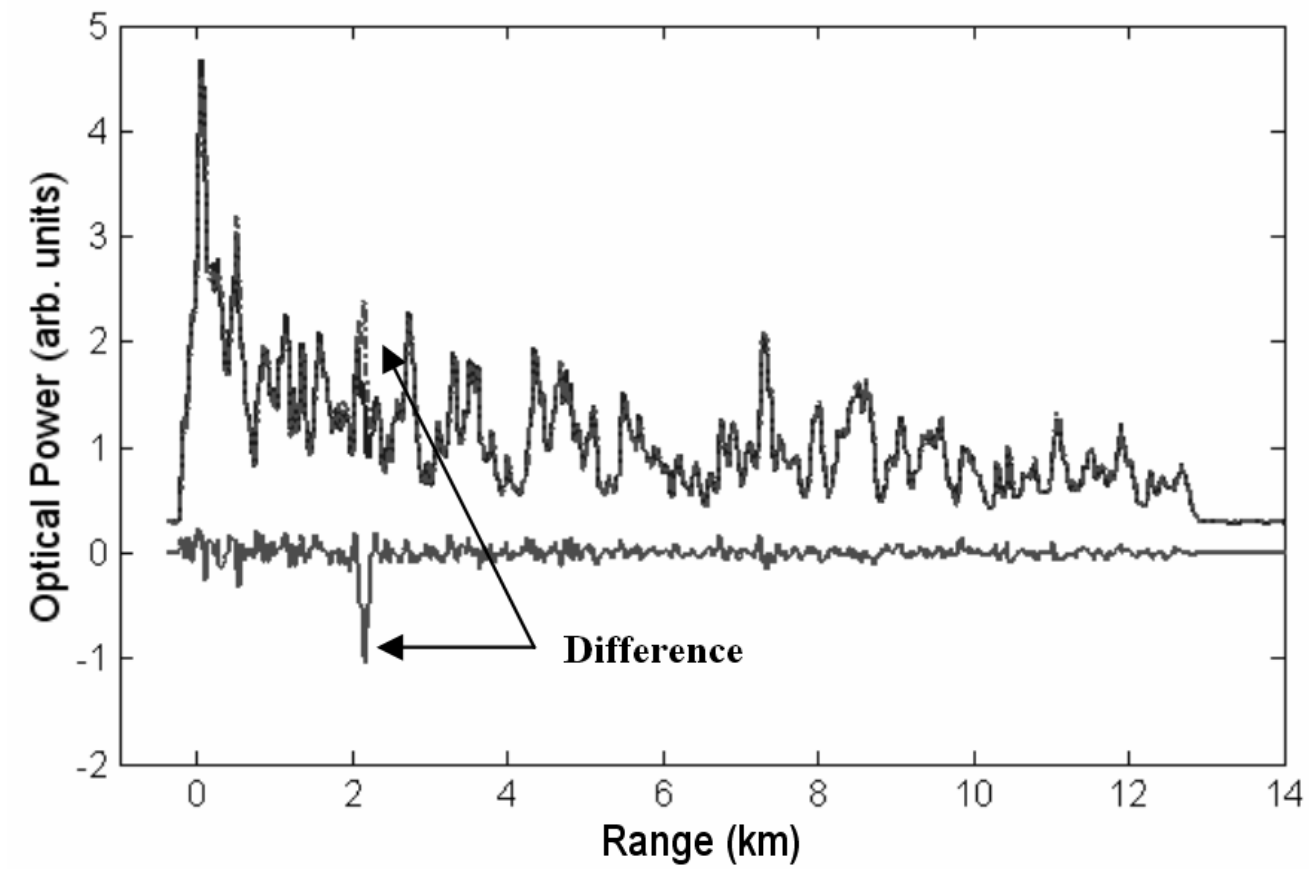

(b)

Figure 36. (a) and (b) $\phi$-OTDR traces for both orthogonal polarizations acquired before and after an $80 \mathrm{~km}$ person has stepped on the ground above the cable along with the difference 


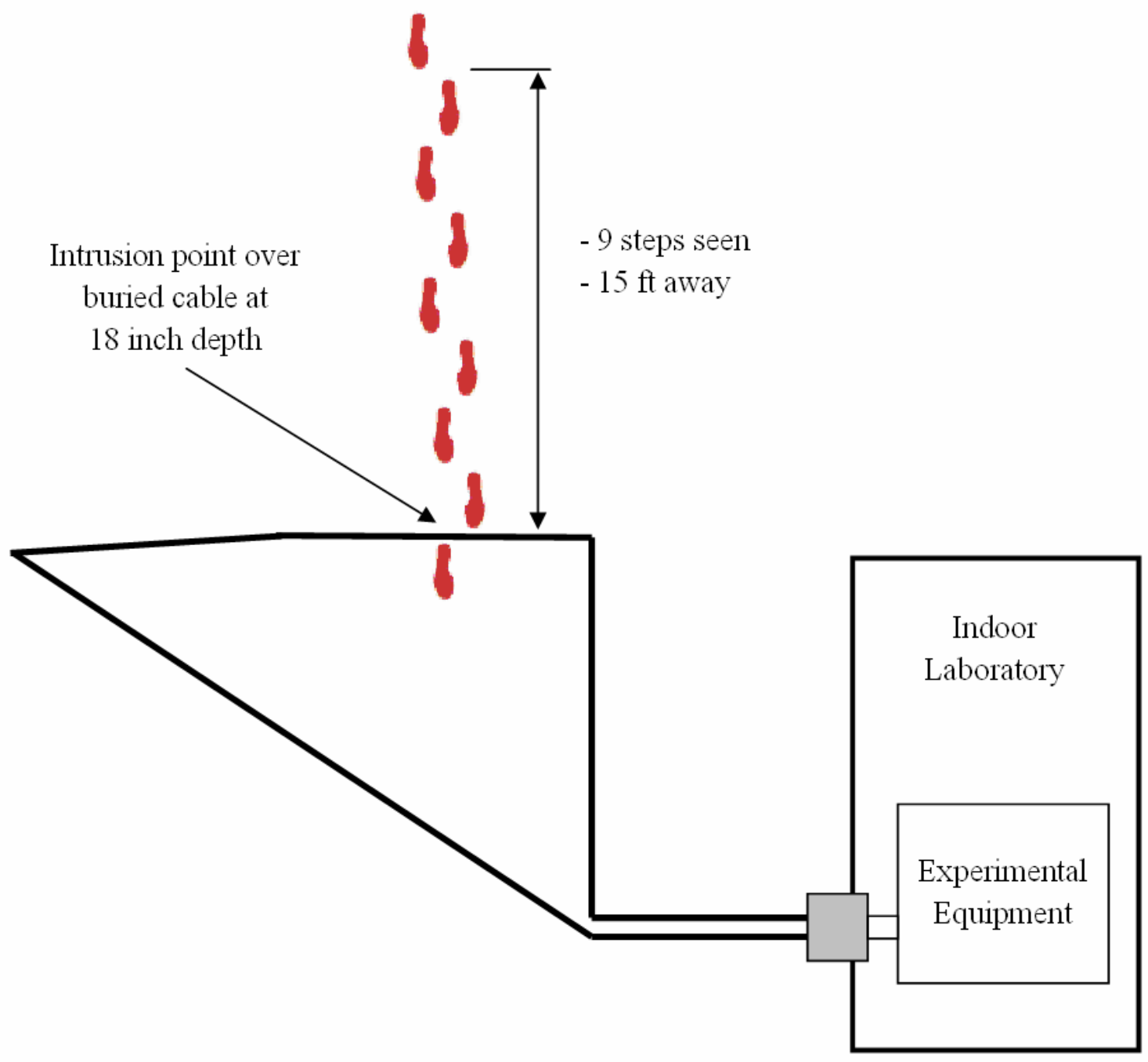

Figure 37. Setup for lateral tests 


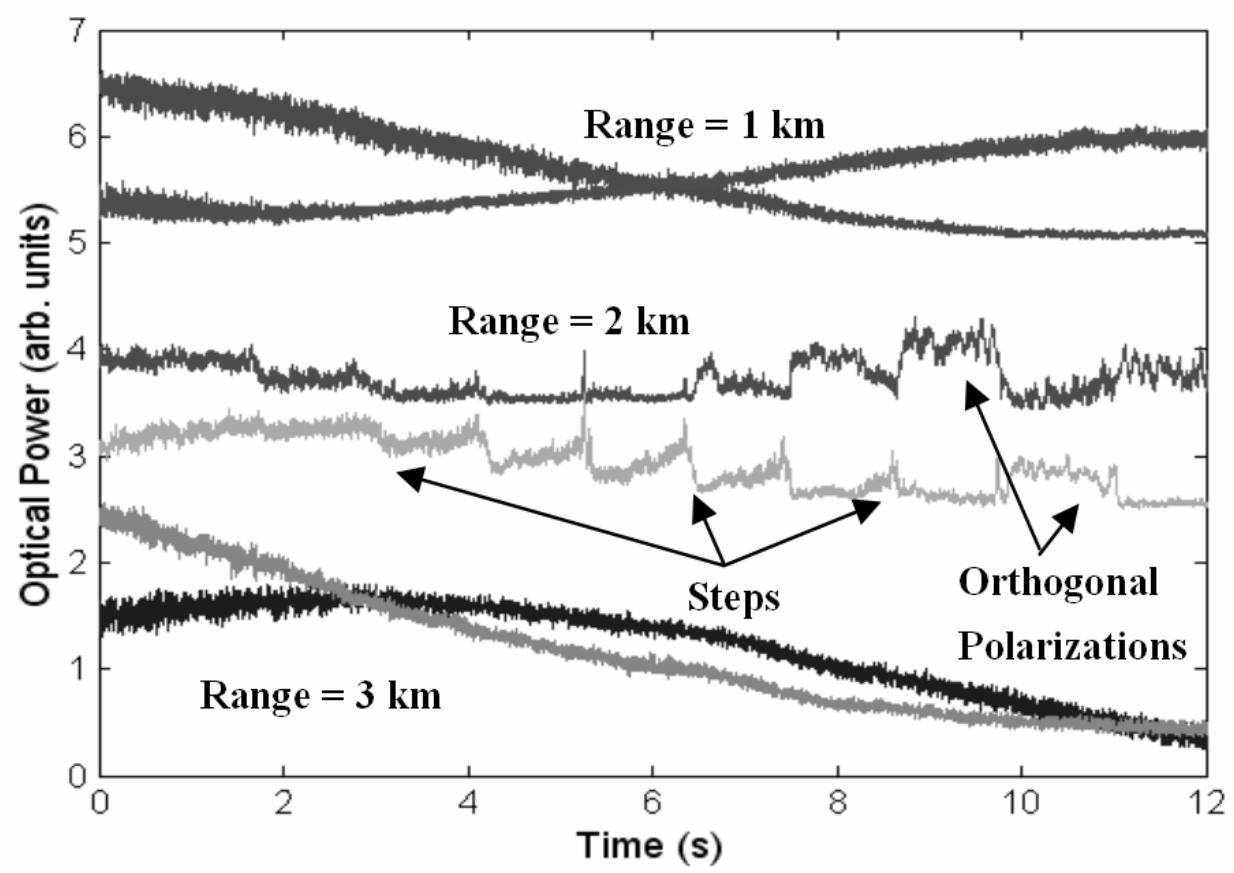

Figure 38. Temporal response of $\phi$-OTDR over 12 seconds at ranges of 1,2 , and $3 \mathrm{~km}$ when a person is approaching the buried fiber cable at the $2 \mathrm{~km}$ location from $15 \mathrm{ft}$ away. 


\section{CHAPTER VIII}

\section{CONCLUSIONS}

A distributed sensor system for detecting and locating intruders based on the phase-sensitive optical time-domain reflectometer ( $\phi-$ OTDR) has been investigated. The light source for the system is a cw Er:fiber Fabry Perot laser with a narrow $(\approx 3 \mathrm{kHz})$ instantaneous linewidth and low (few $\mathrm{kHz} / \mathrm{s}$ ) frequency drift. Phase changes along the length of the fiber are sensed by subtracting a $\phi-$ OTDR trace from an earlier stored trace.

In laboratory tests with fiber on reels, the effects of localized phase perturbations induced by a piezoelectric fiber stretcher on $\phi-$ OTDR traces were observed. In the initial field tests in clay soil of a system in which the sensing element is a 3-mm diameter cabled single mode fiber buried along a monitored perimeter, phase shifts of several- $\pi$ radians produced by people walking on the ground above the buried cable were observed.

In desert terrain field tests in which the sensing element is $8.5 \mathrm{~km}$ of $4.5-\mathrm{mm}$ diameter outdoor fiber optic cable buried in a $1 \mathrm{ft}$ deep, $2.5 \mathrm{ft}$ wide trench filled with loose sand, high sensitivity and consistent detection of intruders on foot and of vehicles traveling down a road near the cable line was achieved in real time. A monitored length of $19 \mathrm{~km}$ was realized by splicing the two fibers at the end of the buried cable in a "loop 
back" arrangement and adding a $2 \mathrm{~km}$ reel of fiber in the building where the monitoring equipment was located.

In a final series of field tests in clay soil, phase changes produced by the steps of a person walking up to $15 \mathrm{ft}$ away from the buried cable were observed. Vehicles traveling at $10 \mathrm{mph}$ were consistently detected up to $300 \mathrm{ft}$ away.

Based on these initial results, this technology may be regarded as a candidate for providing low-cost perimeter security for nuclear power plants, electrical power distribution centers, storage facilities for fuel and volatile chemicals, communication hubs, airports, government offices, military bases, embassies, and national borders. 


\section{CHAPTER IX}

\section{RECOMMENDATIONS}

Various aspects of the intrusion sensor system can be further investigated for improved performance and possible real world application. These include further study into the lateral sensitivity of a buried cable due to soil type, burial depth, and signal fading, the classification of intruders, and the effects of the Erbium doped fiber laser to the sensor's capabilities

Additional field testing is necessary to measure the lateral sensitivity and establish confidence levels of detection by the sensor. Some relationship is expected between lateral sensitivity, burial depth, and soil type. Testing with an all fiber MachZehnder interferometer can provide insight into this relationship and help determine ideal burial depths and soil types for desired lateral sensitivity ranges and confidence levels. Additionally, fading effects in the sensor are expected to cause decreased sensitivity. Study of this phenomenon can help better understand how to improve these nulls in sensitivity.

The ability to classify intruders is a desired feature of this sensor system. With additional testing of varying types of intruders (e.g. humans, vehicles, animals, etc), the unique characteristics of each intrusion signal can be studied. Understanding these characteristics is necessary to develop an advanced signal processing algorithm and a database of signatures that will be necessary to classify intruders in real world applications. 
Lastly, the Erbium fiber laser, the key element to the system, can be improved to produce better system performance. Preliminary tests indicate that operation at lower pump powers minimizes mode hopping and the tendency to emit in multiple longitudinal modes. Other experiments have shown that feedback can have both positive and negative effects on the level of coherence and the amount of mode hopping in the laser. Further study into this can help produce a laser with minimal mode hopping and ensure that it operates in a single longitudinal mode. 


\section{REFERENCES}

[1] M. K. Barnoski and S. M. Jensen, "Fiber waveguides: a novel technique for investigating attenuation characteristics," Appl. Opt., vol. 15, pp. 2112-2115, 1976.

[2] B. Costa and B. Sordo, "Experimental study of optical fiber attenuation by a modified backscattering technique," in Dig. of 3rd Eur. Conf. on Optical Communication (ECOC), 1977, pg. 69.

[3] M. K. Barnoski, M. D. Rourke, S. M. Jensen, and R. T. Melville, "Optical time domain reflectometer," Appl. Opt., vol. 16, pp. 2375-2380, 1977.

[4] H. F. Taylor and C. E. Lee, "Apparatus and method for fiber optic intrusion sensing," U. S. Patent \# 5194 847, issued Mar. 16, 1993.

[5] S. V. Shatalin, V. N. Treschikov, and A. J. Rogers, "Interferometric optical timedomain reflectometry for distributed optical-fiber sensing," Appl. Opt., vol. 37, pp. 5600-5604, 1998.

[6] W. Seo, "Fiber optic intrusion sensor investigation," Ph.D. dissertation, Dept. of Elect. Eng., Texas A\&M University, College Station, TX, 1994.

[7] J. Park and H. F. Taylor, "Fiber optic intrusion sensor using coherent optical time domain reflectometer," Jpn. J. Appl. Phys., vol. 42, pp. 3481-3482, 2003.

[8] K. N. Choi and H. F. Taylor, "Spectrally stable Er:fiber laser for application in phase-sensitive optical time-domain reflectometry," IEEE Photon. Technol. Lett., vol. 15, pp. 386-389, Mar. 2003.

[9] J. P. Dakin, D. A. J. Pearce, A.P. Strong, C. A. Wade, “ A novel distributed fibre sensing system enabling location of disturbances in a Sagnac loop interferometer," in Proc. SPIE, vol. 838, 1987, pp. 325-328.

[10] E. Udd, "Sagnac distributed sensor concepts," in Proc. SPIE, vol. 1586, 1991, pp. 46-52.

[11] M. Campbell, G. Zheng, P. A. Wallace, A. S. Holmes-Smith, " A distributed stress sensor based on a birefringent fiber Sagnac ring," in Proc. SPIE, vol. 2838, 1996, pp. 138-142. 
[12] A. A. Chtcherbakov, P. L. Swart, S. J. Spammer, "Dual wavelength SagnacMichelson distributed optical fiber sensor," in Proc. SPIE, vol. 2838, 1996, pp 301-307.

[13] X. Fang, "Fiber-optic distributed sensing by a two-loop Sagnac interferometer," Opt. Lett., vol. 21, pp. 444-446, 1996.

[14] X. Fang, "A variable-loop Sagnac interferometer for distributed impact sensing," J. Lightw Technol., vol. 14, pp. 2250-2254, Oct. 1996.

[15] S. J. Russell, K. R. C. Brady, J.P. Dakin, "Real-time location of multiple timevarying strain disturbances, acting over a $40-\mathrm{km}$ fiber section, using a novel dualSagnac interferometer," J. Lightw Technol., vol. 19, pp. 205-213, Feb. 2001.

[16] K. N. Choi, J. C. Juarez, and H. F. Taylor, "Distributed fiber-optic pressure/seismic sensor for low-cost monitoring of long perimeters," in Proc. SPIE, vol. 5090, 2003, pp. 134-141.

[17] A. H. Hartog and M. P. Gold, "On the theory of backscattering in single modefiber optical fibers," J. Lightw Technol., vol. 2, pp. 76-82, Apr. 1984.

[18] Corning, (2005, 12 Apr). Basic Principles of Fiber Optics, Corning Cable Systems. Available: http://www.corningcablesystems.com/web/college /fibertutorial.nsf/ofpara

[19] J. C. Palais, Fiber Optic Communications. Upper Saddle River, NJ: Prentice Hall, 1998.

[20] B. Danielson, "Optical time domain reflectometer specifications and performance testing," Appl. Opt., vol. 24, pp. 2313-2321, 1985.

[21] P. Healy, "Review of long wavelength single-mode optical fiber reflectometry techniques," J. Lightw Technol, vol. 3, pp. 876-886, Aug. 1985.

[22] J. Park, "Buried fiber optic sensor," M.S. thesis, Dept. of Elect. Eng., Texas A\&M University, College Station, TX 1992.

[23] A. Havstead, Y. Xie, A. B. Sahin, Z. Pan, A. E. Willner, and B. Fischer, "Delayed self-heterodyne interferometer measurement of narrow linewidth fiber lasers," in Conf. Lasers Electro-Optics (CLEO), May 2000, pp. 310-311.

[24] T. Okoshi, K. Kikuchi, and A. Yakayama, "Novel method for high resolution measurement of laser output spectrum," Electron. Lett., vol. 16, pp. 630-631, 1981. 
[25] Y. Cheng, J. T. Kringlebotn, W. H. Loh, R. I. Laming, and D. N. Payne, "Stable single-frequency traveling-wave fiber loop laser with integral saturable-absorberbased tracking narrow-band filter," Opt. Lett., vol. 20, pp. 875-877, 1995.

[26] G. Bonfrate, F.Vaninetti, and F. Negrisolo, "Single-frequency MOPA Er ${ }^{3+} \mathrm{DBR}$ fiber laser for WDM digital telecommunication systems," IEEE Photon. Technol. Lett., vol. 10, no. 8, pp. 1109-1111, Aug. 1998.

[27] C.-C. Lee, Y.-K. Chen, and S.-K. Liaw, "Single-longitudinal mode fiber laser with a passive multiple-ring cavity and its application for video transmission," Opt. Lett., vol. 23, pp. 358-360, 1998. 


\section{APPENDIX A}

\section{EQUIPMENT USED}

\section{GENERAL}

\section{Electronic:}

Oscilloscope: Tektronix 11201A Digitizing Oscilloscope

Pulse Generators: Tektronix PG501 and PG501 Pulse Generators

Function Generator: Hewlett Packard 3325B

Low Noise Amplifier: Stanford Research Systems SR560

PZT: APC International 42-1080

Current Source (for Pump Laser): ILX Lightwave LDX-3207B

\section{Optical:}

Optical Amplifier \#1: Keopsys Fiber Amplifier KPSBTC13SDFA

Electro Optic Modulator (EOM): JDSU 10023828

Photodetector (for OTDR): ThorLabs PDA400 Amplified InGaAs Detector Photodetector (for MZIs): ThorLabs D400FC InGaAs Detector

Fiber Spools: Corning SMF28

Couplers - 50/50: AC Photonics WP15500102B2011

Couplers - 90/10: AC Photonics WP15100102B2011

WDM Coupler - 980/1550: AC Photonics DP95000102B2100

Isolators - Dual Stage: AC Photonics IU15P21B11 
Circulators: AC Photonics PIOC-15P2111

Fiber Bragg Gratings (FBG) - 1555.4 nm: Avensys (Bragg Photonics) - Custom Erbium Doped Fiber - 18 dB/m: CorActive High-Tech Inc EDF-C 1400

Fiber Polarization Controller (for JDSU EOM): FPC030

\section{Chapter IV \& V: Laboratory Simulation of Sensor and Initial Field Tests}

Electronic:

DAQ Cards: Gage Applied CompuScope 6012

\section{Optical:}

Optical Amplifier \#2: In House Built with EOM Module (OA-2005)

Cable (Buried) - 3-mm diameter (indoor):

\section{Chapter VI \& VII: Yuma Field Tests \& Final Field Tests}

\section{Electronic:}

DAQ Cards: Gage Applied CompuScope 1250 and National Instruments PCI-6111

DAQ BNC Connector Block: National Instruments BNC 2110

Laser Diode Controller (Current/Temperature): ILX Lightwave LDC-3744B

Arbitrary Function Generator: Agilent 33220A

\section{Optical:}

Pump Laser: JDSU 29-8000-360-FL 
Electro Optic Modulator: EOSpace SW-2x2-DOO-SFU-SFU

Polarization Beam Splitter (PBS): Micro-Optics, Inc. PDM-IL-1550

Cable (Buried) - $4.5 \mathrm{~mm}$ diameter (outdoor): OCC 


\section{APPENDIX B}

\section{EQUIPMENT CONNECTION SCHEMATICS}

\section{Chapter IV: Schematic of Electronic Equipment for OTDR in Laboratory Experiments}

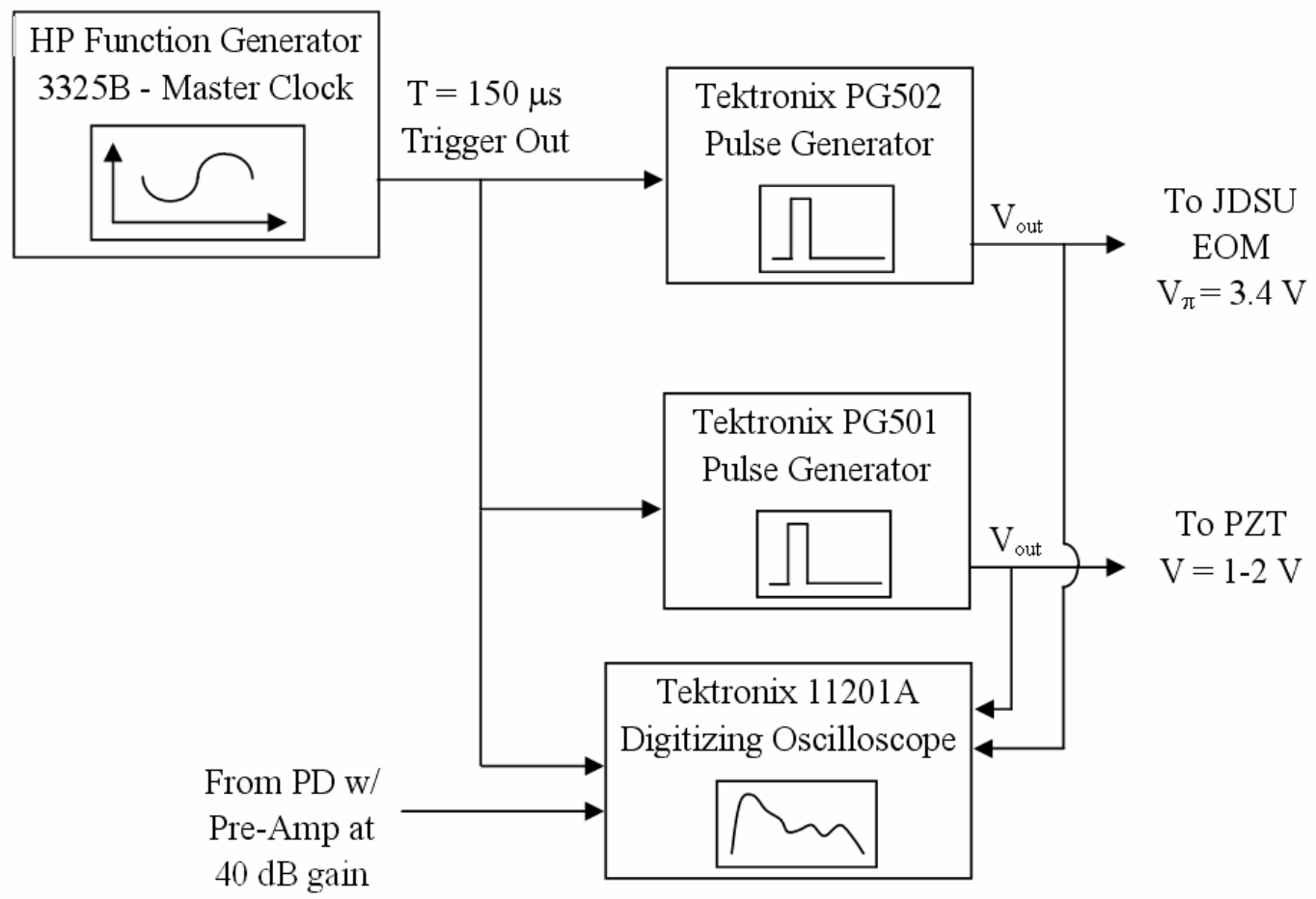

\section{Notes:}

- Master Clock was set to $150 \mu$ s period

- Tektronix PG501 Pulse Generator was set to $10 \mu$ s pulse width and external triggering

- In 3 trace experiment (Figure 19), Tektronix PG502 Pulse Generator was set to $150 \mu$ s pulse width and external trigger

- In ramp voltage experiment (Figure 21), the Tektronix PG502 was replaced with a function generator outputting a $\sim 100 \mathrm{~Hz}$ sinusoidal signal

- Tektronix 11201A Digitizing Oscilloscope triggered by Master Clock and operated in DC coupling, $20 \mathrm{MHz}$ Bandwidth, $1 \mathrm{M} \Omega$ Impedance

- ThorLabs Photodetector D400FC operated with $40 \mathrm{~dB}$ gain 


\section{Chapter V: Schematic of Electronic Equipment for OTDR in Field Tests, Series 1, Brazos County, TX}

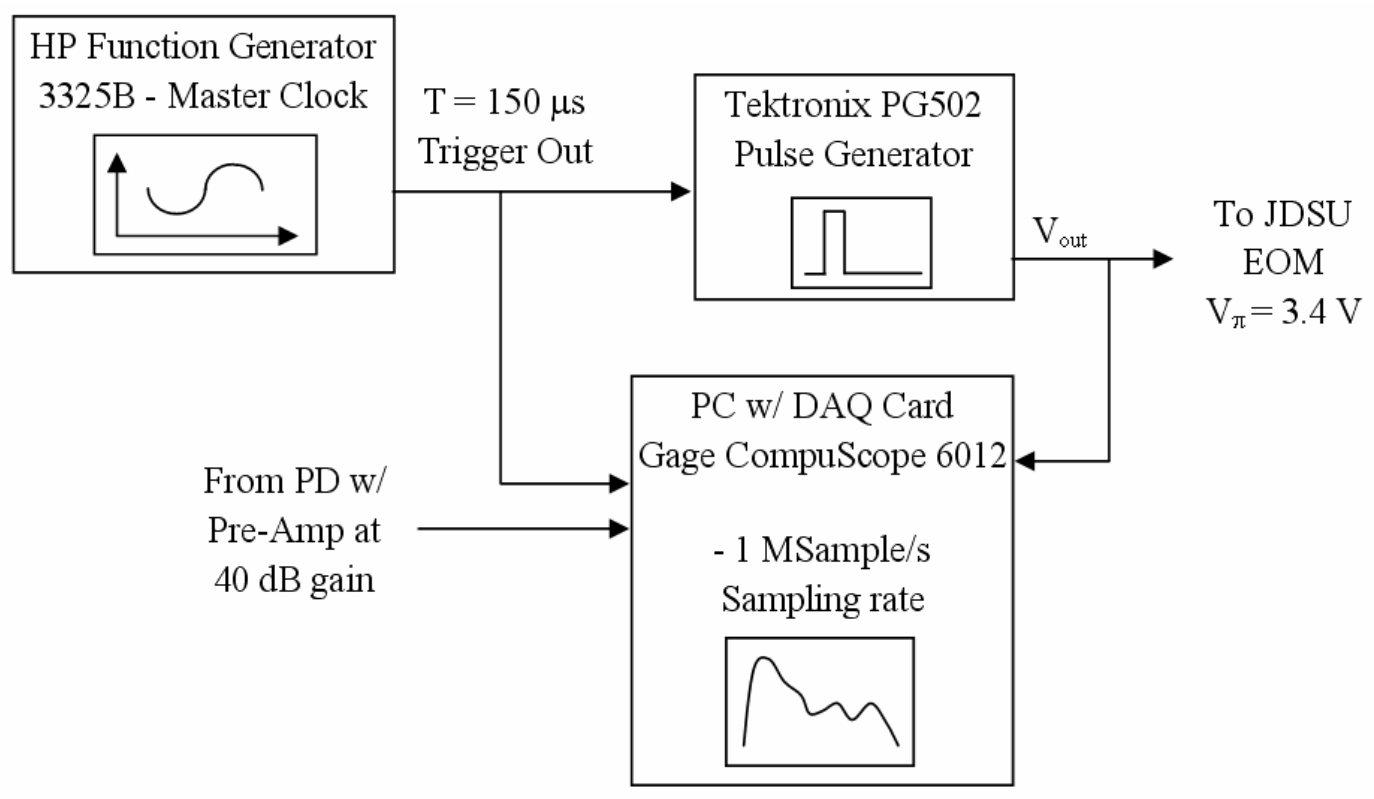

Notes:

- Master Clock was set to $150 \mu$ s period

- Tektronix PG501 Pulse Generator was set to $10 \mu$ s pulse width and external triggering

- Tektronix 11201A Digitizing Oscilloscope triggered by Master Clock and operated in DC coupling, $20 \mathrm{MHz}$ Bandwidth, $1 \mathrm{M} \Omega$ Impedance

- ThorLabs Photodetector D400FC operated with $40 \mathrm{~dB}$ gain

- Gage DAQ Card was ( ) and used and ISA port on the PC, which resulted in slow transfer from the board memory to the PC memory 


\section{Chapter VI: Schematic of Electronic Equipment for OTDR in Field Tests, Series 2, Yuma, AZ}

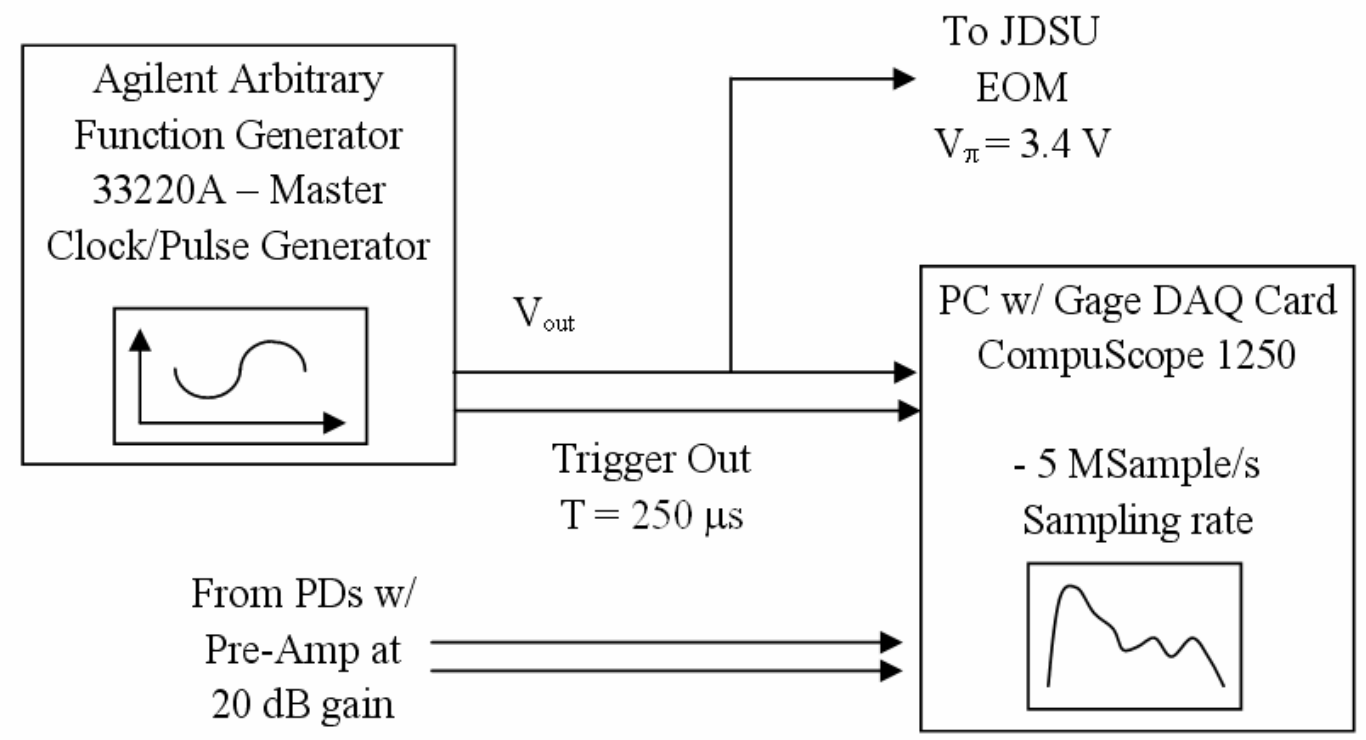

Notes:

- Agilent Function Generator was set to a $250 \mu$ s period and $2 \mu$ s pulse width

- Both Gage and NI DAQ Card were set to $5 \mathrm{MHz}$ sampling rate, DC coupling, and $1 \mathrm{M} \Omega$ Impedance

- ThorLabs Photodetectors D400FC operated with $20 \mathrm{~dB}$ gain 


\section{Chapter VII: Schematic of Electronic Equipment for OTDR in Field Tests, Series 3, Brazos County, TX}

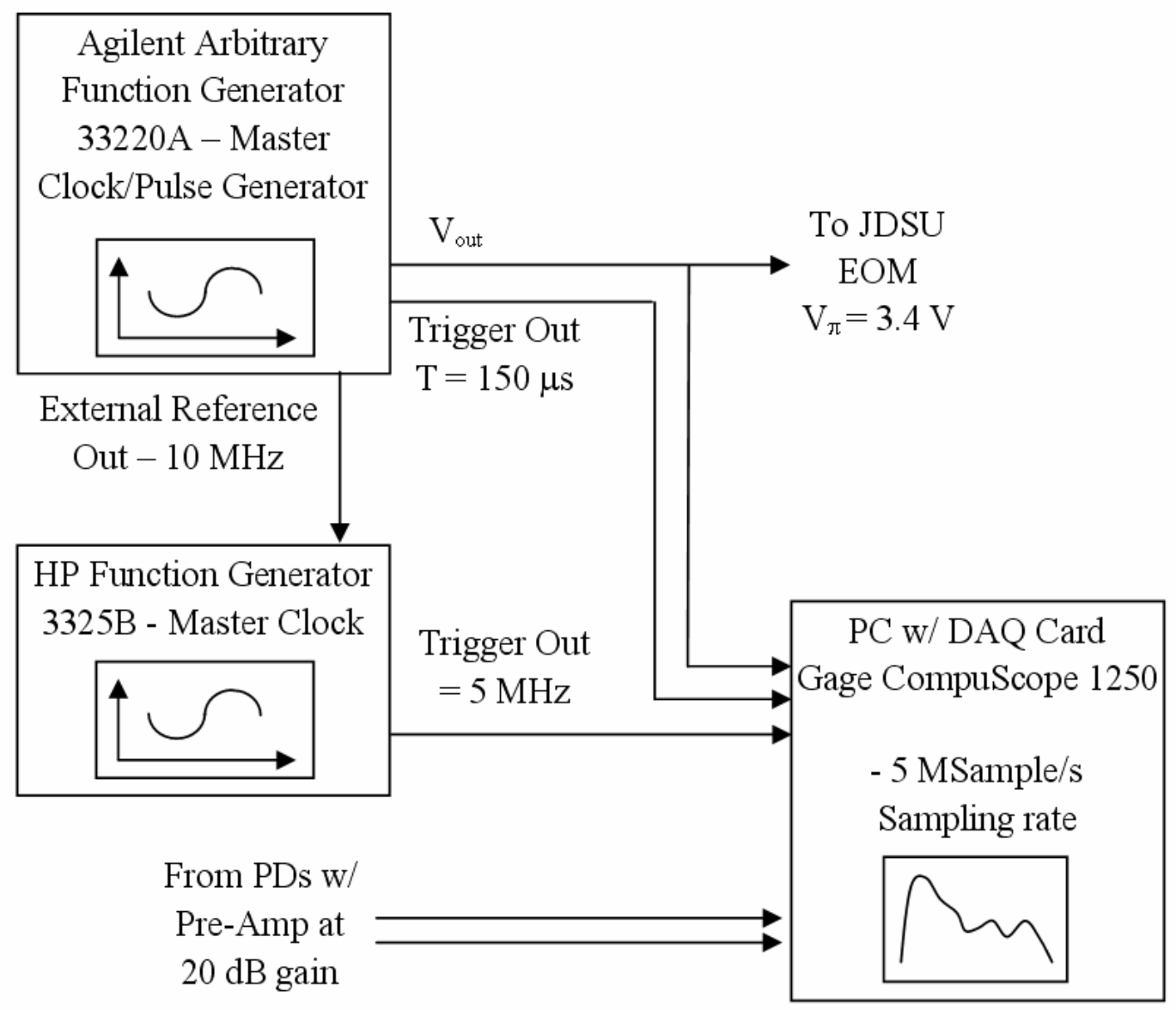

Notes:

- Agilent Function Generator was set to a $150 \mu$ s period and $2 \mu$ s pulse width

- Agilent Function Generator External Reference Out (10 MHz) was connected to External Reference In of HP Function Generator to synchronize them

- HP Function Generator Trigger Out was set to $5 \mathrm{MHz}$ and was used to set the sampling rate of the NI DAQ Card via the external clock feature in order to synchronize acquisition to the EOM pulse

- NI DAQ Card was set to DC coupling, and $1 \mathrm{M} \Omega$ Impedance

- ThorLabs Photodetectors D400FC operated with $20 \mathrm{~dB}$ gain 


\section{VITA}

Mr. Juan C. Juarez was born in Laredo, Texas in 1978. He received his Bachelor of Science and Master of Science degrees in electrical engineering from Texas A\&M University, College Station, TX, in 2000 and 2002, respectively.

Immediately after graduation, he continued his studies in the Department of Electrical Engineering at Texas A\&M University by beginning his doctorate work under the direction of Dr. Henry F. Taylor. He has been working as a Research Assistant since June of 2000. In the summer of 2001, he worked for the Product Development Group of Corning Cable Systems testing and developing hardware and software for a low-cost directional coupler test bench. In the summer of 2003, he worked in the Nonproliferation and International Security Division of Los Alamos National Laboratory studying VCSELs for use with a Quantum Key Distribution system. His research interests are in the fields of fiber optic sensors, fiber lasers, VCSELs, and quantum encryption systems.

Mailing Address: Department of Electrical Engineering

214 Zachry Engineering Center

3128 TAMU

College Station, TX 77845-3128 